NSMRL/50904/TR--2014-1310 October 01, 2014

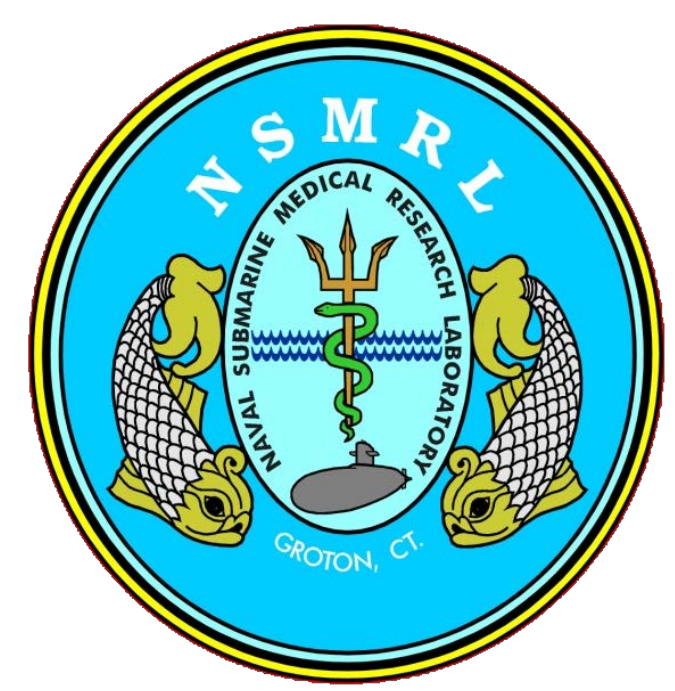

Three Methods for Estimating the Middle-Ear Muscle Reflex (MEMR) Using Otoacoustic Emission (OAE) Measurement Systems

by:

Judi A Lapsley Miller

Lynne Marshall

Naval Submarine Medical Research Laboratory

Approved and Released by:

S. M. WECHSLER, CAPT, MC, USN

Commanding Officer

NAVSUBMEDRSCHLAB

Approved for public release; distribution unlimited 



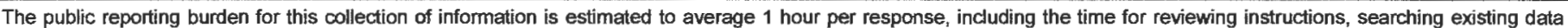

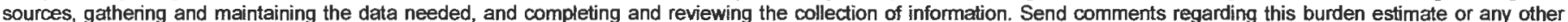

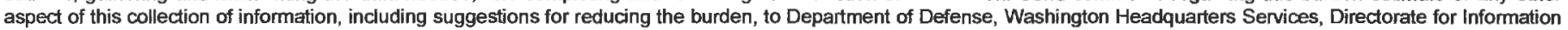

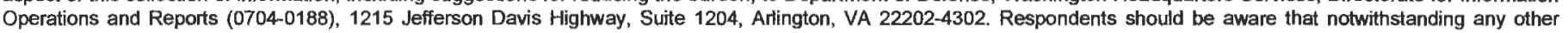
provision of law, no person shall be subject to any penalty for failing to comply with a collection of information if it does not display a currently valid OMB control number. PLEASE DO NOT RETURN YOUR FORM TO THE ABOVE ADDRESS.

\begin{tabular}{l|l|l|l}
\hline $\begin{array}{l}\text { 1. REPORT DATE (DD-MM-YYYY) } \\
01 / 10 / 2014\end{array}$ & $\begin{array}{l}\text { 2. REPORT TYPE } \\
\text { Technical Report }\end{array}$ & $\begin{array}{l}\text { 3. DATES COVERED (FrOm - To) } \\
2007-2014\end{array}$ \\
\hline
\end{tabular}

\section{TITLE AND SUBTITLE}

THREE METHODS FOR ESTIMATING THE MIDDLE-EAR MUSCLE

REFLEX (MEMR) USING OTOACOUSTIC EMISSION (OAE)

5a. CONTRACT NUMBER

MEASUREMENT SYSTEMS

5b. GRANT NUMBER

ONR N0001412WX20904

5c. PROGRAM ELEMENT NUMBER

5d. PROJECT NUMBER

5e. TASK NUMBER

5f. WORK UNIT NUMBER

50904

7. PERFORMING ORGANIZATION NAME(S) AND ADDRESS(ES)

Naval Submarine Medical Research Laboratory

6. AUTHOR(S)

Judi A. Lapsley Miller

Lynne Marshall

8. PERFORMING ORGANIZATION REPORT NUMBER

NSMRL/50904/TR-2014-1310

9. SPONSORING/MONITORING AGENCY NAME(S) AND ADDRESS(ES)

Office of Naval Research

10. SPONSORMONITOR'S ACRONYM(S)

ONR

11. SPONSOR/MONITOR'S REPORT NUMBER(S)

12. DISTRIBUTION/AVAILABILITY STATEMENT

Approved for public release; distribution unlimited

13. SUPPLEMENTARY NOTES

14. ABSTRACT

A sensitive measure of the middle-ear muscle reflex (MEMR) is needed when making other sensitive audiological tests where activating the MEMR could confound and confuse test results. In this report we document and describe three related methods for making this sensitive MEMR measurement using the OAE and MOCR measurement modules in the Mimosa Acoustics HearlD system. All three methods can sensitively detect small MEMR; however, for many ears, test variability is too high to make a clear determination of MEMR threshold.

\section{SUBJECT TERMS}

Middle-ear muscle reflex, audiology, hearing, acoustic reflex

16. SECURITY CLASSIFICATION OF:

\begin{tabular}{|l|l|l|l|}
\hline a. REPORT & b. ABSTRACT & c. THIS PAGE & \\
ABSTRACT \\
& $U$ & $U$ & SAR \\
\hline
\end{tabular}

18. NUMBER 19 a. NAME OF RESPONSIBLE PERSON

OF
PAGES

68
NAVSUBMEDRSCHLAB Commanding Officer 19b. TELEPHONE NUMBER (Include area code) 860-694-3263 
[THIS PAGE INTENTIONALLY LEFT BLANK 


\title{
Three Methods for Estimating the Middle-Ear Muscle Reflex (MEMR) Using Otoacoustic Emission (OAE) Measurement Systems
}

\author{
Judi A. Lapsley Miller \\ Lynne Marshall \\ Naval Submarine Medical Research Laboratory
}

\section{Naval Submarine Medical Research Laboratory}

\author{
Approved and Released by:

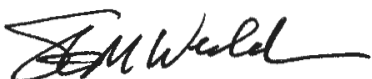 \\ S. M. WECHSLER, CAPT, MC, USN \\ Commanding Officer \\ Naval Submarine Medical Research Laboratory \\ Submarine Base New London Box 900 \\ Groton, CT 06349-5900
}

Administrative Information

The work was supported by ONR Grant/Agreements N0001407WX20658 (NSMRL work unit number 50517) and N0001412WX20904 (NSMRL Work Unit number 50904). The views expressed in this report are those of the authors and do not necessarily reflect the official policy or position of the Department of the Navy, Department of Defense, nor the United States Government. Lynne Marshall is an employee of the U.S. Government. This work was prepared as part of her official duties. Title 17 U.S.C. $\$ 105$ provides that 'Copyright protection under this title is not available for any work of the United States Government.' Title 17 U.S.C. $\$ 101$ defines a U.S. Government work as a work prepared by a military service member or employee of the U.S. Government as part of that person's official duties.

Approved for public release; distribution unlimited 
[THIS PAGE INTENTIONALLY LEFT BLANK 


\begin{abstract}
A sensitive measure of the middle-ear muscle reflex (MEMR) is needed when performing other sensitive audiological tests where activating the MEMR could confound and confuse test results. One example is when measuring another ear reflex - the medial olivocochlear reflex (MOCR) - which requires detecting small changes in evoked otoacoustic emissions (OAEs). Current clinical MEMR tests using immittance have proven insensitive for these applications, with minimum detectable thresholds of around $70 \mathrm{~dB}$ SPL. For sensitive MOCR measurements, MEMR activity in the range of 55-65 dB SPL needs to be reliably detectable. One method to estimate the MEMR magnitude and threshold more sensitively is to monitor changes in an OAE-evoking stimulus to a MEMR-inducing contralateral broadband noise, which changes the magnitude and phase of the OAE-evoking stimulus. By simultaneously suppressing the OAE, an estimate of the MEMR can be derived, unconfounded by the OAE or the MOCR. In this report we document and describe three related methods for making this sensitive MEMR measurement using the OAE and MOCR measurement modules in the Mimosa Acoustics HearID system. All three methods can sensitively detect small MEMR; however, for many ears, test variability is too high to make a clear determination of MEMR threshold. Examples from three experiments show a variety of test outcomes, including simultaneous MEMR and MOCR measurements. In summary, the benefits to these methods are that the MEMR test is sensitive; it can be derived from the MOCR test; and it can be made relatively simply using commercially-available OAE systems. However, clinical implementations and applications of these methods are not yet viable due to the high inherent trial-to-trial variability seen in many ears. Alternative MEMR tests should continue to be pursued, especially those that can track the change in stimulus level faster.
\end{abstract}

\title{
ACKNOWLEDGEMENTS
}

Thanks to Paul Boege for his insights and help with the underlying calculations. Thanks to Charlotte Reed, Zachary Perez, and Tim Villabona at Massachusetts Institute of Technology (MIT) for their assistance with data collection and methodology. Thanks to John Guinan and Chris Shera for suggesting the OAE-based method for making sensitive MEMR measurements. 


\section{Contents}

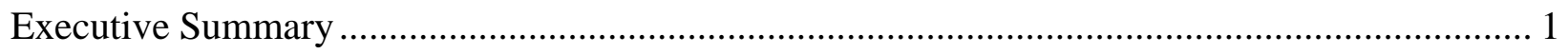

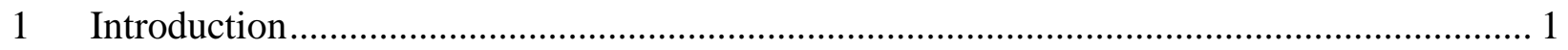

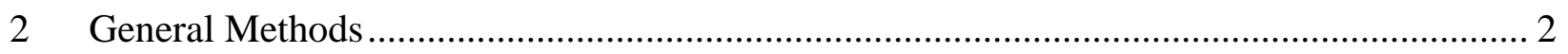

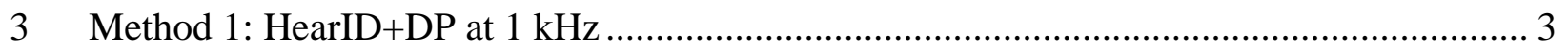

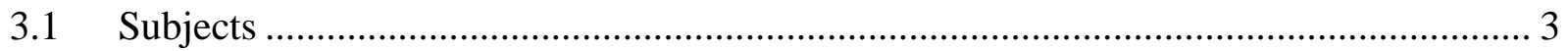

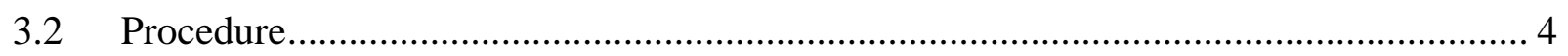

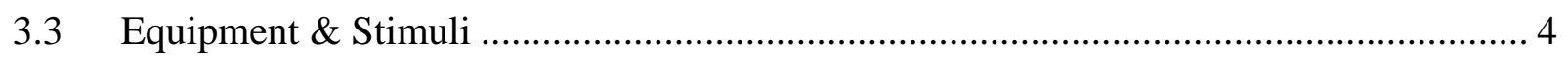

3.4 Data Analysis Overview......................................................................................... 5

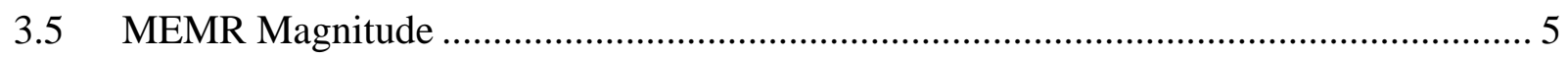

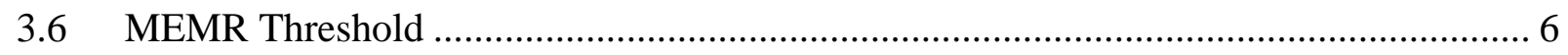

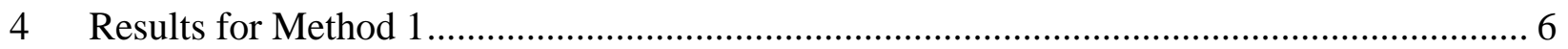

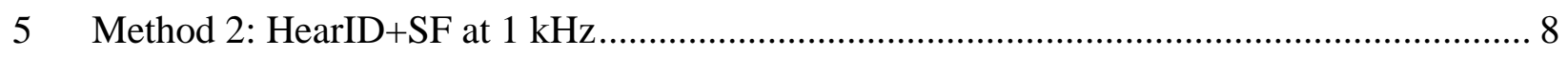

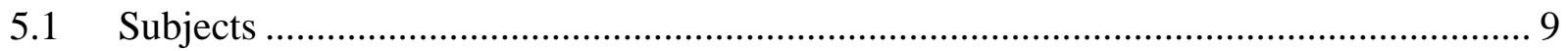

5.2 Equipment \& Stimuli ............................................................................................ 9

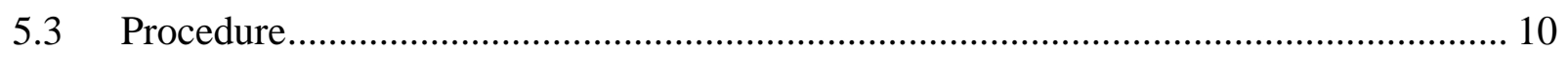

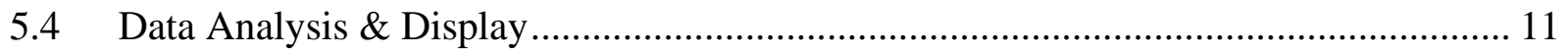

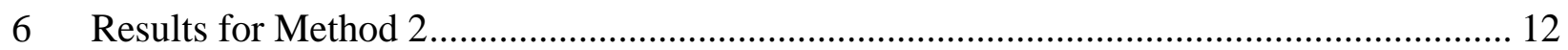

7 Method 3: HearID+MOCR at the MOCR test frequency ..................................................... 13

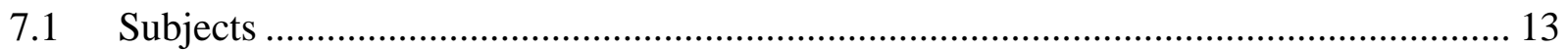

7.2 Equipment \& Stimuli ........................................................................................... 13

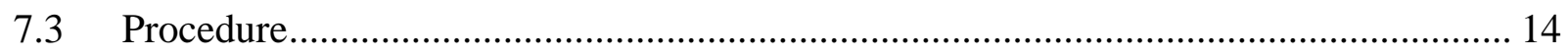

7.4 Data cleaning and initial processing .......................................................................... 14

7.5 Deriving MEMR from MOCR measurements ........................................................... 14

7.6 MEMR Analysis and screening criteria ……………................................................. 15

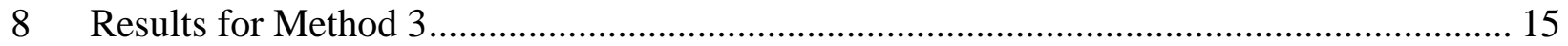

8.1 Summarizing the MEMR analyses......................................................................... 16

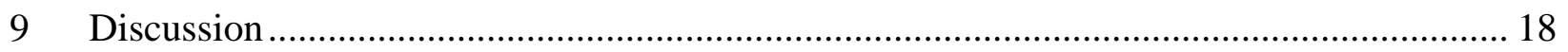

9.1 Source of variability ........................................................................................... 19

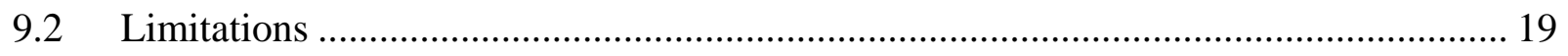

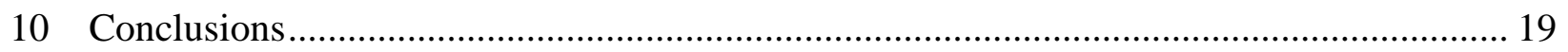




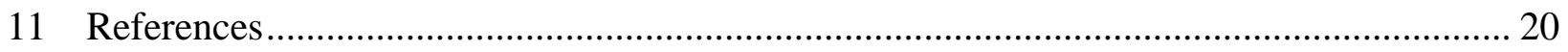

12 Appendix A: List of Acronyms...................................................................................... 22

13 Appendix B: Method 1 examples of MEMR magnitude .................................................... 23

14 Appendix C: Method 2 examples of MEMR magnitude and threshold ............................. 26

14.1 MEMR with clearly identifiable MEMR threshold................................................ 26

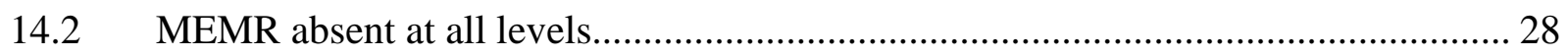

14.3 MEMR appears to be present, but is not detectable from variability ....................... 29

14.4 MEMR present at higher levels, ambiguous at lower ........................................... 30

14.5 Ambiguous results - Variability High even when Noise is low.............................. 32

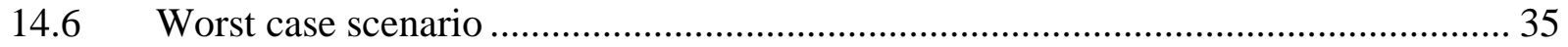

15 Appendix D: Method 3 MOCR and MEMR.................................................................... 36

16 Appendix E: Method 3 MOCR and MEMR box plots ............................................... 42 


\section{Executive Summary}

Issues: a sensitive test for the middle-ear muscle reflex (MEMR) magnitude and threshold is needed for both laboratory studies and clinical application.

Objective: refine a method for testing the MEMR using clinical otoacoustic emission (OAE) equipment.

Approach: changes in a tonal stimulus presented in the test ear canal to a contralateral MEMRelicitor were measured while simultaneously suppressing the otoacoustic emission (OAE) generated by the stimulus. Three similar approaches were developed, all based on equipment usually used for making OAE and medial-olivocochlear reflex (MOCR) measurements.

Results: three sensitive methods for estimating the MEMR magnitude and threshold were developed. Two methods also allowed simultaneous measurements of OAEs and MOCR. However, all methods showed high trial-by-trial variability in around one-third of subjects reducing the ability to reliably and validly detect the presence of a small MEMR, thus limiting the application of the method to the laboratory.

\section{Introduction}

The middle-ear muscle reflex (MEMR) is an involuntary sound-evoked contraction of the stapedius muscle. This contraction pulls the stapes away from the oval window, stiffens the ossicular chain and the tympanic membrane, and decreases the acoustic power flow through the middle ear, especially at $1 \mathrm{kHz}$ and below. The MEMR is also known as the acoustic reflex or stapedial reflex. The MEMR can be evoked by ipsilateral, contralateral, or bilateral stimulation.

The MEMR threshold (the lowest sound level that evokes a MEMR response) varies widely across individuals, and is commonly measured clinically as part of a differential diagnosis for many hearing conditions. However, estimates of the MEMR threshold using standard clinical tympanometers are insensitive, and can overestimate the threshold (e.g., Zhao \& Dhar, 2010). For applications where a low MEMR threshold could affect or confound the measurement of another property, a more sensitive estimate of MEMR threshold is needed. Such applications include many sensitive hearing-system measurements (e.g., Buki, Wit, \& Avan, 2000), including otoacoustic emissions (OAEs), wideband acoustic immittance (WAI), and medial-olivocochlear reflex (MOCR) tests. All these tests underpin our hearing-conservation research program at NSMRL.

The MEMR can confound some hearing-system measurements because the acoustic stimuli used may evoke a MEMR response in the same frequency region as the measurement. By estimating the MEMR threshold for an individual ear, we can set the acoustic stimulus to a level below that which could evoke a MEMR. To achieve this requires measuring MEMR activity at much lower levels than what is needed for clinical diagnostics. But there is no commercial or clinical system that directly makes and reports these more sensitive measurements. There are, however, many laboratory implementations because it is a ubiquitous problem in hearing research (e.g., Feeney \& Keefe, 2001; Goodman \& Keefe, 2006; Goodman, Mertes, Lewis, \& Weissbeck, 2013; Guinan, Backus, Lilaonitkul, \& Aharonson, 2003; Henin, Long, \& Thompson, 2014; Lilaonitkul \& Guinan, 2009a, 2009b; Luebke, Stagner, Martin, \& LonsburyMartin, 2014; Xu, Cheatham, \& Siegel, 2014). Here we consider the method outlined by Lilaonitkul and 
Guinan (2009a, 2009b), where they detected MEMR more sensitively by looking for systematic changes in a tonal stimulus using a bespoke laboratory system. This approach was promising for us because a variation could be implemented on the commercial OAE systems we were using in our experiments, without buying additional equipment or software. The purpose of this report is to document the methodology we have used since 2007 with Mimosa Acoustics’ HearID systems (Champaign, IL).

We had two aims:

1. Develop an OAE-system-based method to make the MEMR measurement for use in our laboratory investigations of the medial-olivocochlear reflex (MOCR).

2. Develop a clinically-feasible MEMR method for eventual use with a clinical MOCR test, and for any other clinical application requiring a more sensitive estimate of the MEMR magnitude or threshold.

Through three major iterations we improved and further automated the methodology. This report documents how we achieved the first aim, but shows that achieving the second aim is still some way off due to variability in many ears that swamps the MEMR measurement.

\section{General Methods}

What is the general concept of our MEMR measurement? Engaging the MEMR stiffens the middle ear, which modifies acoustic power flow. This can change the stimulus tone measured in the ear canal. We monitor amplitude and phase changes in the stimulus tone in the ear canal as a contralateral broad band noise (BBN), which is the MEMR activator, is turned on and off. The stimulus tone in the ear canal can be monitored without interference from the evoked stimulus-frequency otoacoustic emission (SFOAE) by suppressing the SFOAE with a second (ipsilateral) tone. See Figure 1 for a simple schematic.

More specifically:

1. A stimulus tone at probe frequency, $f_{p}$, is played at a specific, calibrated level in the test ear canal (typically around $1 \mathrm{kHz}$ and $40 \mathrm{~dB}$ SPL). This tone may also generate an OAE at $1 \mathrm{kHz}$. Both the stimulus and the OAE are measurable in the ear canal.

2. Simultaneously, a suppressor tone, $f_{s}$, at a nearby frequency (within $\sim 100 \mathrm{~Hz}$ ) and a much higher level (typically around $60 \mathrm{~dB}$ SPL) is also played at a specific, calibrated, level in the test ear. $f_{s}$ suppresses the OAE at $f_{p}$, leaving only the stimulus, $f_{p}$, measurable in the ear canal. ${ }^{1}$

3. $f_{p}$ and $f_{s}$, alone or in combination, are not intense enough to evoke the MEMR.

4. In alternating trials, a broadband noise contralateral elicitor is turned on and off in the other ear. This is the MEMR-evoking stimulus. When it is turned on, the MEMR may engage.

5. The change in $f_{p}$ may be in magnitude, phase, or both. By calculating the vector difference (i.e., change in phase and magnitude) of $f_{p}$ with and without the contralateral elicitor, an estimate of the

\footnotetext{
${ }^{1}$ The suppressor tone also may generate an OAE at $f_{s}$, but it does not affect the measurement at $f_{p}$. The combined tones of $f_{p}$ and $f_{s}$ also create distortion product OAEs, measurable at predictable frequencies such as $2 f_{p}-f_{s}$, etc., but again they can be safely ignored because we are only measuring MEMR effects at $f_{p}$.
} 
size of the MEMR effect can be derived. It too has phase and magnitude, but we are interested mostly in the magnitude, which is expressed in $\mathrm{dB}$ SPL.

6. To establish the MEMR threshold, the contralateral BBN level is systematically varied (e.g., from 50 to $90 \mathrm{~dB}$ SPL in $5 \mathrm{~dB}$ steps). The lowest level at which a change is reliably detected is considered threshold.

7. Simultaneously, estimates of measurement variability may be made.

8. For the parameters we typically use, MEMR magnitude typically varies across ears from approximately $-5 \mathrm{~dB}$ SPL to $15 \mathrm{~dB}$ SPL, for contralateral BBN levels 55 to $70 \mathrm{~dB}$ SPL.

The following methods and results sections describe three implementations of this general method.

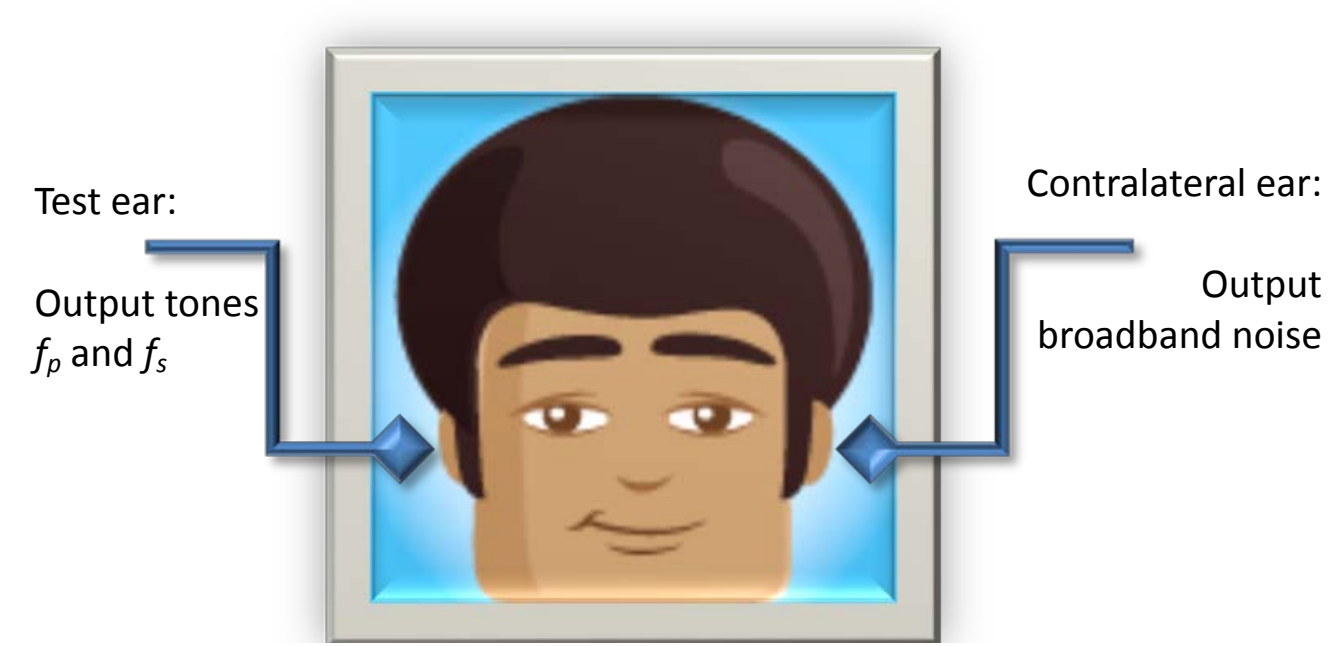

Figure 1. Schematic showing which ear receives which stimulus during a MEMR measurement. $f_{p}$ is the probe tone, and $f_{s}$ is the suppressor tone.

\section{Method 1: HearID+DP at $1 \mathrm{kHz}$}

To estimate the MEMR threshold, a series of OAE measurements were made in the test ear where the $\mathrm{OAE}$ at $1 \mathrm{kHz}$ was continually suppressed by the suppressor tone. By suppressing the OAE that is evoked by the stimulus tone (at the same frequency as the stimulus tone), only the stimulus tone is measured in the ear canal. Thus the stimulus tone may be monitored for changes due to the MEMR. Five pairs of measurements with and without a contralateral MEMR-eliciting stimulus were made. Vector changes of the responses at $1 \mathrm{kHz}$ were calculated, and because the OAE itself was suppressed, any change in the response was primarily due to the activation of the middle-ear muscles. Activation of the MOCR could not be seen due to suppression of the OAE. Four contralateral BBN noise levels were used: 50, 55, 60, and $65 \mathrm{~dB}$ SPL. The lowest BBN stimulus that evoked a change larger than $0 \mathrm{~dB}$ SPL was considered the MEMR threshold for that ear.

\subsection{Subjects}

The 115 subjects were adults (61\% female, 39\% male) aged 18-40 years old, with normal hearing, and no known auditory pathology. These subjects received the MEMR test as part of the screening battery used to determine if they met study eligibility for OAE experiments. All testing was conducted at the Massachusetts Institute of Technology (MIT). The experimental protocol was approved by that institute's 
Committee on the Use of Humans as Experimental Subjects (COUHES) and conducted in compliance with regulations and ethical guidelines on experimentation with human subjects..

\subsection{Procedure}

Before each measurement series, the Mimosa Acoustics' HearID system calibrated the stimulus level in-the-ear using a wideband chirp. If the tester was satisfied with the calibration, they started the measurement series. HearID allows 10 measurements to be made in one measurement session using the same calibration. We used this feature to run a series of measurements alternating with and without the contralateral elicitor, in the order QN-QN-QN-QN-QN where Q is a quiet trial and $\mathrm{N}$ is a contralateral noise trial. A three second pause was used between each measurement to allow the reflex to reset. Each contralateral BBN level was tested in a separate measurement series, starting from the lowest level. Testing 4 levels took around 20 minutes.

It was not possible to directly monitor noise levels at $f_{p}$, and the initial version of HearID we were using had a bug that meant the noise level at the nearby $2 f_{1}-f_{2}$ was not shown so the tester was operating somewhat blindly.

\subsection{Equipment \& Stimuli}

Measurements were made using the distortion-product otoacoustic emission (DPOAE) module of HearID R3.2 (pre-release) or R3.3 (custom modified) systems (Mimosa Acoustics, Inc.). HearID used the 24-bit digital-signal-processing "Echo" PC-card, running on an IBM Think Pad T43 laptop computer. Tonal stimuli were delivered and responses recorded with an ER10C probe (Etymōtic Research, Inc.) using foam eartips.

The contralateral broadband noise $(0.01$ to $10 \mathrm{kHz})$ was generated by an analog noise generator (Grason Stadler Corporation, Model 901B), attenuated by a programmable attenuator (Tucker-Davis Technologies, Inc., Model PA4), amplified with an integrated stereo amplifier (Realistic SA-150, Model 31-1955), and output to an ER-2 tubephone (Etymōtic Research, Inc.). The timing of the noise was controlled manually by the tester.

The DPOAE measurement normally outputs two tones simultaneously at $f_{1}$ and $f_{2}$, which are separated by the ratio $f_{2} / f_{1}=1.2$. The DPOAE itself is reported at frequency $2 f_{1}-f_{2}$. It was possible to modify the DPOAE test parameters to change the frequency ratio and levels to something more suitable for measuring the MEMR. HearID saves the DPOAE data into an Access database, including the stimulus magnitude and phase at $f_{p}\left(f_{1}\right.$ in the DPOAE module). This information was accessed using the Matlab Database toolbox. At the time, we chose to use the DPOAE module of HearID, rather than Mimosa Acoustic's SFOAE-SG system, because it was not possible to set the suppressor stimulus in the SFOAE module to continually suppress the SFOAE (i.e., it cycled on and off). However, this meant we could not monitor the noise level at $f_{p}$.

Stimulus parameters

- $f_{1}$ tone $=0.984 \mathrm{kHz}$ (which resolved to $0.9821429 \mathrm{kHz}$ ), $40 \mathrm{~dB}$ SPL

- $f_{2}$ tone $=1.031 \mathrm{kHz}$ (which resolved to $1.03125 \mathrm{kHz}$ ), $60 \mathrm{~dB}$ SPL

- $f_{2} / f_{1}$ ratio $=1.05$

- Stimulus duration = 3 seconds (which is overridden if artifact rejection/stopping rules is turned on)

- Minimum DPOAE and signal to noise ratio (SNR) were both set to $30 \mathrm{~dB}$, to essentially disable them 
- Minimum DPOAE and signal to noise ratio (SNR) were both set to $30 \mathrm{~dB}$, to essentially disable them

Artifact rejection was turned on, with parameters:

- Noise level criterion for rejection in data frame = $10 \mathrm{~dB}$ SPL (same as factory default)

- Noise level above calibration noise floor criterion for rejection in data frame $=10 \mathrm{~dB}$ (same as factory default)

Stopping rules were modified to ensure they did not become activated during the protocol:

- Noise Floor Level for Accepting distortion product (DP) Measured = -20 dB SPL, which effectively disables this criterion as we were not interested in the noise floor at the DP frequency

- Acceptable DP to Noise Floor Ratio $=40 \mathrm{~dB}$, which effectively disables this criterion as we were not interested in the DPOAE.

- Maximum Signal Duration for a Frequency Point = 3 seconds, set to ensure that all stimulus presentations were an equal duration.

\subsection{Data Analysis Overview}

For each BBN level, the ten $f_{p}$ stimulus levels and phases were plotted in polar coordinates and visually inspected for signs that the group of measurements without the contralateral stimulus was different from the group of measurements with the contralateral stimulus. In most cases, the two groups were indistinguishable from the test-retest variability. Lilaonitkul and Guinan (2009a, 2009b) did not explicitly provide a quantification for their method for detecting the MEMR. Post hoc, we developed the following method. MEMR magnitude was defined for each measurement pair (with and without the contralateral elicitor) as the vector change at the stimulus frequency $f_{\mathrm{p}}$ in $\mathrm{dB}$ SPL. To gauge whether this reflex magnitude (for each contralateral elicitor level) was detectable from measurement drift, noise, and variability over the five trial pairs, the same calculation was made with repairings of the trials without a contralateral elicitor (trials 1-3, 3-5, 5-7, and 7-9). This can be thought of as the inherent variability of the test. Box and whisker plots of the two distributions were used to ascertain whether the MEMR magnitudes were clearly different from the inherent variability, and whether the MEMR magnitudes were greater than a (rather arbitrary) $0 \mathrm{~dB}$ SPL. A good measurement series always had inherent variability below $0 \mathrm{~dB}$ SPL. Very small MEMRs were difficult to distinguish from measurement variability in some subjects.

There are two measurements of interest: the MEMR magnitude (at a single BBN level) and the MEMR threshold (from the four BBN levels).

\subsection{MEMR Magnitude}

The $f_{p}$ stimulus level, $L_{p}$, magnitude (dB SPL) and phase (radians) measured in the ear canal is stored in the HearID database. Using a Matlab script, these numbers were transformed into their complex number representation, after converting the magnitude from decibels into Pascals using the transform, $10^{\left|L_{p}\right| / 20}$. For each QN pair, the MEMR magnitude was estimated by taking the vector difference between the two complex levels: $L_{p Q}-L_{p N}$ from the Q and N trials to get $M_{p}$. This magnitude was transformed into dB SPL: $20 \times \log _{10}\left|M_{p}\right|$.

Five estimates of the MEMR were obtained from the five trial pairs, for which there could be variability and systematic drift. To gauge the validity and reliability of the MEMR estimates, an estimate of the MEMR inherent variability was made for comparison. There was no estimate of noise floor at $f_{p}$ 
with this system. To estimate the variability, the same calculation as above was made but on repairings of the Q trials: Q1-Q3, Q3-Q5, Q5-Q7, Q7-Q9, giving four estimates of the MEMR variability.

If the MEMR variability was at a similar or higher level to the MEMR magnitude, then we did not consider the MEMR detectable. To be considered valid and large enough to be of interest, the MEMR magnitude had to be greater than $0 \mathrm{~dB}$ SPL and distinct from the noise.

This method takes some probe drift into account, and also possibly over-estimates the variability, because drift affects the QQ repairings more than the QN pairings, which are closer together in time.

\subsection{MEMR Threshold}

To estimate the threshold, the box and whisker plots as a function of contralateral stimulus level were plotted on the same graph. In general, as the contralateral stimulus level increases, the MEMR tends to increase too and be distinct from the noise. We defined threshold as the lowest contralateral BBN level at which the MEMR was at least $0 \mathrm{~dB}$ SPL in magnitude and distinct from the noise.

\section{$4 \quad$ Results for Method 1}

An example measurement (5 trial-pairs) at one contralateral BBN level is shown in Figure 2. The plot on the left shows the stimulus level in the complex Cartesian plane after converting to complex numbers (as described above). Blue symbols represent $\mathrm{Q}$ trials and red symbols represent $\mathrm{N}$ trials. When eyeballing the data, a MEMR is detectable if the blue symbols are separated from the red symbols.

On the right are box and whisker plots, but note that the MEMR is based on 5 pairs and the variability on 4 pairs. In this example, there clearly is a MEMR. The red line of the box represents the median, the blue box is the interquartile range, and the red crosses indicate outliers. The whiskers represent the range of points within 1.5xIQR.

Further examples are shown in the Appendix on page 23. 

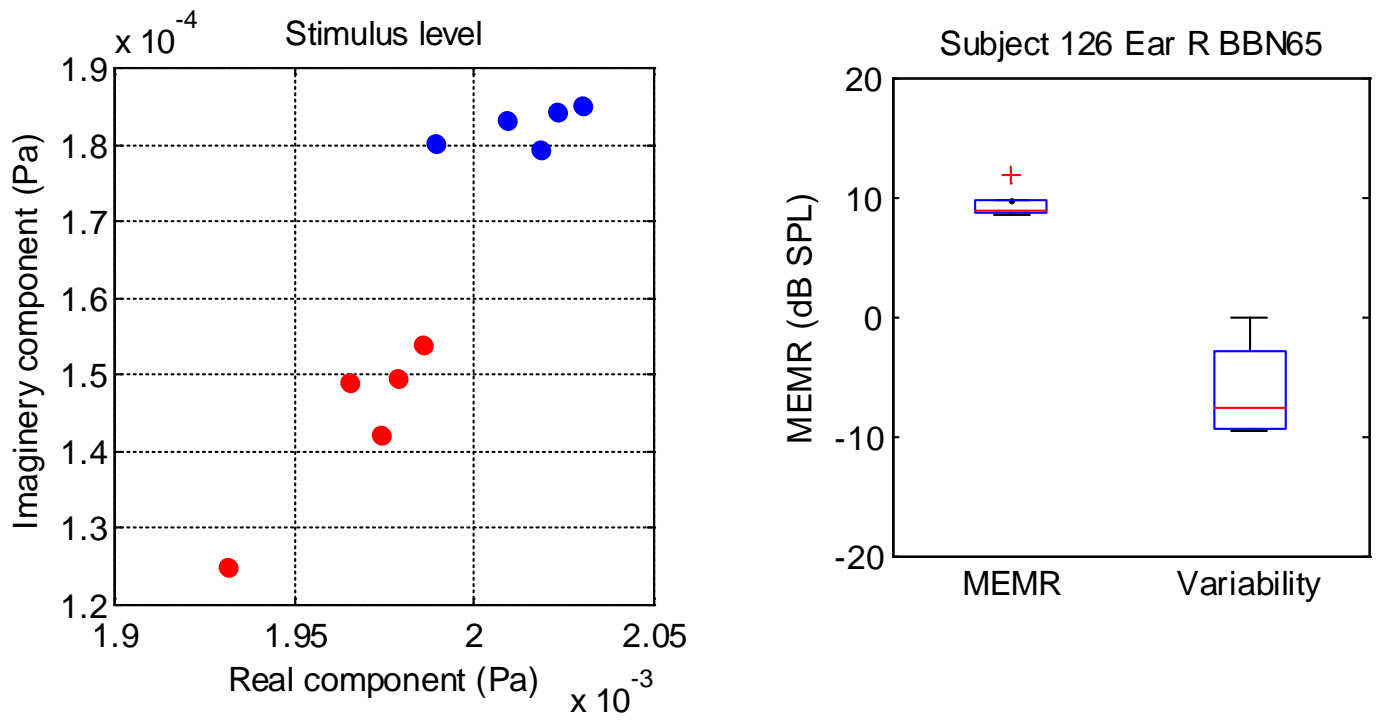

Figure 2. Example MEMR measurement. (left) stimulus level in Pascals showing clear separation between $Q$ trials in blue and $\mathrm{N}$ trials in red, (right) box plot of the derived MEMR of around $10 \mathrm{~dB}$ SPL that is detectable from the inherent variability in the test (see text for description).

To estimate the threshold, the box and whisker plots as a function of contralateral stimulus level were plotted on the same graph (Figure 3 shows an example). In general, as the contralateral stimulus level increases, the MEMR tends to increase too and be distinct from the noise. In Figure 3, the reflex at $60 \mathrm{~dB}$ SPL is small in magnitude, but distinct. There is a clear reflex at $65 \mathrm{~dB}$ SPL. In comparison, Figure 4 shows no MEMR at any level. Note that the levels we are testing are far lower than those normally tested clinically, and many subjects showed no MEMR activity.

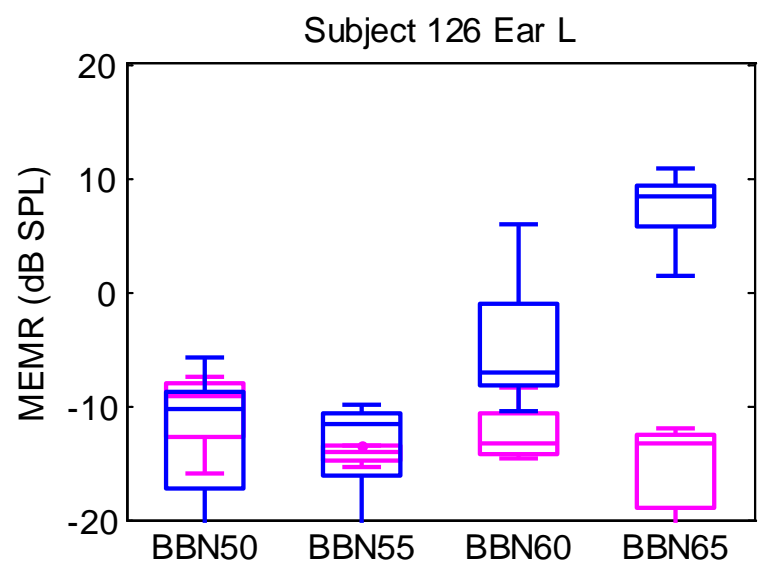

Figure 3. The four box-plots show the MEMR magnitude (blue) as the BBN level increases from 50 to $65 \mathrm{~dB}$ SPL. The variability in the measurement (pink) is low. There is a distinct MEMR response at 60 and $65 \mathrm{~dB}$ SPL, so the threshold is considered to be $60 \mathrm{~dB}$ SPL. 


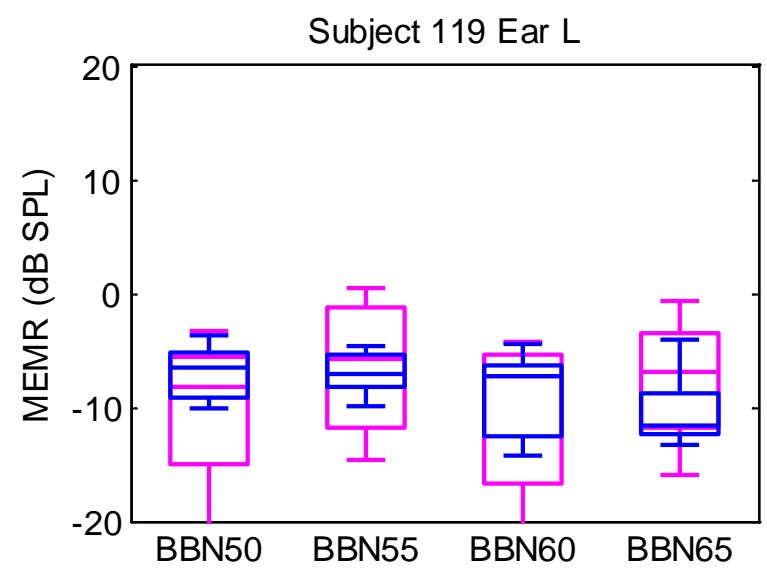

Figure 4. No MEMR at any of the four levels tested (see Figure 3 for plot details).

Method 1 was used in the development of an MOCR assay for use in humans (Marshall et al., in press). During initial subject screening for study eligibility, the results for the MEMR test were plotted in polar coordinates and visually examined for evidence of a reflex. After the experiment finished, we quantified the method as described above and reanalyzed the 30 subjects who otherwise passed screening. Three subjects were excluded because we could not determine their MEMR threshold with sufficient reliability. This method was also used in a subsequent experiment, from which the examples in Figure 2-4 and Appendix B are drawn.

\section{$5 \quad$ Method 2: HearID+SF at $1 \mathrm{kHz}$}

In Method 1, we used a modified DPOAE test to estimate the MEMR; however, the process was very labor intensive and we had little information or control over noise levels. In Method 2, we used the HearID+SF system. This version allowed for noise rejection and noise levels reported at $f_{p}$, semiautomated timing, digital contralateral noise, and automated file saving. The same information obtained in Method 1 regarding the changes in stimulus magnitude and phase at $f_{p}$ can be extracted from part of the SFOAE measurement.

SFOAEs can be measured at $f_{p}$ using two tones: the probe tone at $f_{p}$ and the suppressor tone at $f_{s}$ (Shera \& Guinan, 1999). The SFOAE is extracted by cycling an ipsilateral tonal SFOAE suppressor at $f_{s}$ on and off and measuring the vector difference in ear canal pressure at $f_{p}$. This separates the SFOAE response at $f_{p}$ from the stimulus at $f_{p}$.

- Variation 1: SFOAEs measurements were made as normal.

- Variation 2: Mimosa Acoustics modified SFOAEs to allow the ipsilateral suppressor at $f_{s}$ to be on for the entire measurement, and not cycled on and off. Thus, there was never an SFOAE to extract because it was always suppressed. We did preliminary work using this variation, but eventually decided to proceed with variation 1 as results were similar and variation 1 allowed us to also extract information about the SFOAE and, therefore, the MOCR for comparison (i.e., to see if the MEMR was affecting the MOCR).

Each SFOAE measurement file contains not just the derived SFOAE but also the pressure for the probe-alone section (Pp) and the pressure for the probe+suppressor section (Pps). This allows us to recombine and reanalyze the information in different ways, post hoc. 
To measure SFOAE MOCR, a series of SFOAE trial pairs are collected: Q and N. Q is a regular SFOAE measurement. $\mathrm{N}$ also has a contralateral BBN elicitor. The BBN elicitor partially suppresses the SFOAE at $f_{p}$ (depending on the MOCR strength), but not the stimulus itself at $f_{p}$. The raw MOCR strength is the vector difference between the SFOAE measured during the $\mathrm{Q}$ and $\mathrm{N}$ trials. It can be converted into $\mathrm{dB}$ SPL and compared with the MEMR which is also reported in $\mathrm{dB}$ SPL.

If the MEMR is activated by the BBN elicitor then both the stimulus and SFOAE response at $f_{p}$ (and $f_{s}$ ) are potentially affected. We can estimate the MEMR magnitude by suppressing the SFOAE at $f_{p}$ while doing a MOCR-style measurement series. Any change in level at $f_{p}$ can only be due to changes in the stimulus level (caused by the MEMR) if the SFOAE itself is suppressed. If we make a normal SFOAEMOCR measurement series (i.e., variation 1 where the $f_{s}$ cycles on and off), we can estimate (a) the MEMR by looking at the difference at Pps (where the SFOAE is always suppressed) between the Q and $\mathrm{N}$ trial, and (b) the MOCR derived from the SFOAEs measured at the same time (Pp - Pps). This way we can see if the MEMR is affecting the MOCR ${ }^{2}$. (Although the MOCR comparison is only meaningful if the SFOAE at $f_{p}$ has sufficient SNR to derive the MOCR).

To pull the comparison out more clearly:

- For MEMR, take the vector difference between Pps_Q and Pps_N (i.e., comparing the same tone with suppressor across two separate SFOAE measurements - one with and one without the contralateral BBN).

- For MOCR, take the vector difference between Pp_Q and Pps_Q (to get SFOAE_Q) and between Pp_N and Pps_N (to get SFOAE_N), then take the difference between the resulting SFOAEs (SFOAE_Q and SFOAE_N), i.e.,

$$
\begin{aligned}
\text { MOCR_SFOAE } & =(\text { Pp_Q }- \text { Pps_Q })-\left(P p \_N-P p s \_N\right) \\
& =\text { SFOAE_Q }- \text { SFOAE_N }
\end{aligned}
$$

- All calculations are done as vector differences on complex numbers.

\section{$5.1 \quad$ Subjects}

The 77 subjects (67\% female, 33\% male) were from an experiment designed to establish stopping rules for MOCR measurements made with an SFOAE test. Subjects were adults aged 18-40 years old, with normal hearing, and no known auditory pathology. These subjects received the MEMR test as part of the screening battery used to determine if they met study eligibility. All testing was conducted at MIT. The experimental protocol was approved by that institute's internal review board and conducted in compliance with regulations and ethical guidelines on experimentation with human subjects. Subjects provided informed consent and were paid for their participation in the experiments.

\subsection{Equipment \& Stimuli}

Mimosa Acoustics HearID+SF+CAS-addon R3.3 and R3.4-beta system was used to make the MEMR measurements. The SFOAE module version varied from 3.1.0 through 3.1.23. The SFOAE module used the SFOAE algorithm from Shera and Guinan (1999). The CAS add-on was purpose-built by Mimosa Acoustics for NSMRL. It provided the ability to semi-automatically control the timing, level, and presentation of a contralateral elicitor through an external sound card (M-Audio transit).

The contralateral elicitor was a $10 \mathrm{kHz}$ low-pass broadband Gaussian noise, digitally generated and output through the 24-bit M-Audio Transit card via an ER2 tubephone. The onset delay between the

\footnotetext{
${ }^{2}$ Thanks to Paul Boege for pointing out that the MEMR measurement was already embedded in the MOCR measurement.
} 
starting of the contralateral noise and the start of the OAE measurement was 2 seconds and the offset delay between the end of the contralateral noise and the start of the next OAE measurement in quiet was at least 3 seconds. This allowed more than enough time for the MEMR to activate and reset. Six contralateral noise levels were tested in increasing intensity: 50, 55, 60, 65, 70, and 75 dB SPL.

The SFOAE measurement parameters were:

- $f_{p}=0.99609 \mathrm{kHz}, 40 \mathrm{~dB}$ SPL

- $f_{s}=1.04300 \mathrm{kHz}, 60 \mathrm{~dB}$ SPL

- $\quad$ noise rejection criterion (wideband noise ) $=48 \mathrm{~dB}$ SPL

Stopping rules were used in most tests to terminate the test early and automatically as soon as the noise level was sufficiently low and at least 4 averages were obtained, otherwise it continued until any of the other criteria were met

- $\quad$ maximum noise level estimated around $f_{p}=-10 \mathrm{~dB}$ SPL

- $\quad$ minimum number of averages $=4$

- $\quad$ maximum number of averages $=16$

- $\quad$ maximum number of rejects $=20$

- $\quad$ maximum test time $=60 \mathrm{~s}$

- minimum SNR = -99 dB (disabled, because we were not interested in the SFOAE)

The stimuli were calibrated using an in-the-ear calibration chirp and levels were monitored during the test. In rare cases, the MEMR effect was so strong that the $f_{p}$ level was considered to be too far from the calibration target level (a clinical implementation of this test would need to override this criterion during $\mathrm{N}$ trials).

\subsection{Procedure}

Five or six Q-N trial-pairs were collected per contralateral BBN level. Unlike Method 1, the tester could run the measurement series to collect as many trial-pairs as they deemed necessary to get quality data. 


\subsection{Data Analysis \& Display}

\section{Subject ID 282, R Ear}
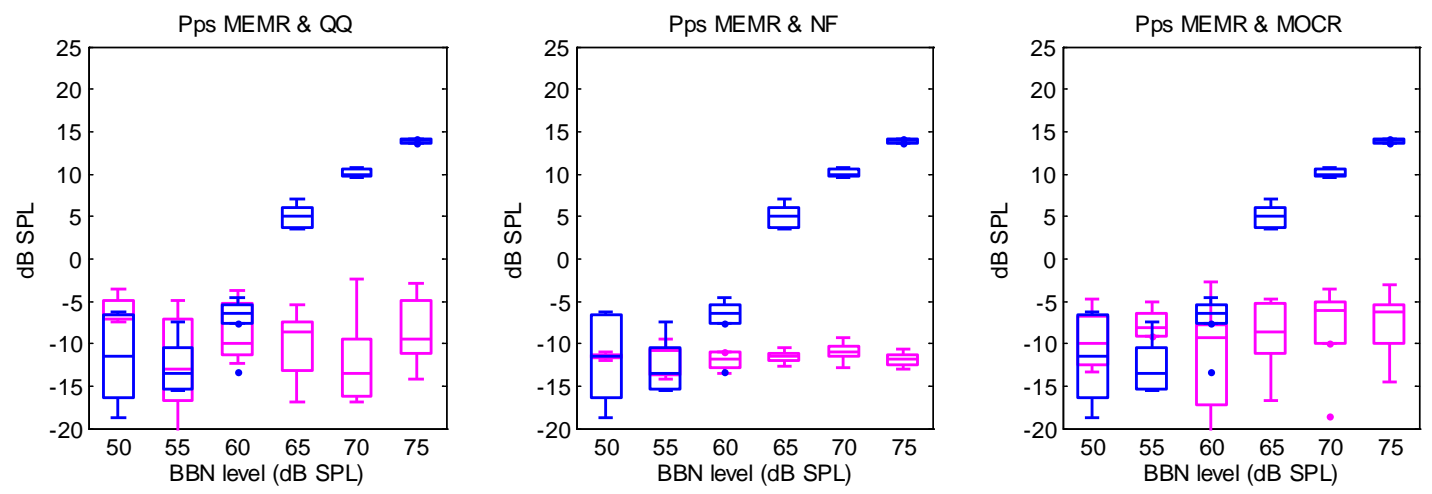

Figure 5. "Perfect" data with MEMR present and low variability and noise. In all three plots, the blue symbols are the MEMR. The pink symbols in the left plot show variability across the quiet trials (no contralateral elicitor), in the middle plot show within-trial noise-floor variability, and in the right plot show the MOCR (see text below for details). For 282R, MEMR (blue) threshold is at $65 \mathrm{~dB}$ SPL and is clearly distinguishable from QQ variability (pink, left) and MEMR noise (pink, middle). A MOCR was not present at $1 \mathrm{kHz}$ (pink, right). At $60 \mathrm{~dB}$ there could be a tiny MEMR, but it is undetectable from QQ variability (pink, left). It is unlikely that the MEMR would affect a MOCR test made with BBN=60 dB SPL

Results were plotted in a three-paneled plot, showing various aspects of the measurement (e.g., Figure 5). The left plot shows the same box-plots as in Method 1. The middle plot shows the same MEMR information, but this time plotted against the noise levels in the measurement (which we now have access to in Method 2). For the reader also interested in the MOCR, the right plot shows how MEMR and MOCR vary as the contralateral level increases (which is only meaningful if the subject has an SFOAE at $1 \mathrm{kHz})$.

In Figure 5 and the Appendix on page 26, the plots show the following information:

- $\quad$ MEMR is always in blue; comparisons are always in pink. All measures are plotted as a function of the BBN contralateral elicitor level in dB SPL.

- MEMR is extracted from the Pps section so is unconfounded with the MOCR and SFOAE, which is suppressed by the $f_{s}$ tone.

- MEMR \& Variability plot The plot on the left compares the MEMR estimates against the trial-bytrial variability, estimated by pairing up adjacent "Q" trials (referred to here as “QQ" variability), as in Method 1. If there is a difference between one $Q$ measurement and the next that is larger than the difference due to the MEMR (which isn't activated in Q trials) then you can't reliably detect the MEMR. To be confident that a MEMR was detected, the MEMR magnitude distribution should be higher than and separated from the QQ variability distribution. ${ }^{3}$ By example, if a 6-trial-pair series is labelled (Q1-N2), (Q3-N4), (Q5-N6), (Q7-N8), (Q9-N10), and (Q11-N12), there are five QQ repairings possible of adjacent Q trials. They are: (Q1-Q3), (Q3-Q5), (Q5-Q7), (Q7-Q9), and (Q9-Q11). There is one fewer QQ trial-pair than there is for the QN series. Note that Q3, Q5, Q7, and Q9 are

\footnotetext{
${ }^{3}$ Note that when we use this technique for MOCR, we use QQ and NN trial-pairings, but for MEMR, the NN trial-pairs are too variable. This is because if the reflex engages, it does not necessarily have the same magnitude each time. So if the adjacent $\mathrm{N}$ trials are paired, there can be a difference just because the MEMR magnitude was different. Whereas for MOCR, the magnitude is far more consistent, so the NN trial-pairs can be used to add to the number of sample used for assessing trial-by-trial variability.
} 
used twice (i.e., compared with the $\mathrm{Q}$ trial before and after it). This is done to extract as much variability information as possible while still having realistic test times.

- MEMR \& Noise plot: The middle plot compares the MEMR estimates to the MEMR "noise" estimated from the noise levels in the two Pps sections from the $\mathrm{Q}$ and $\mathrm{N}$ trials and calculated similarly to the SFOAE noise floor (i.e., where the Pp and Pps noise levels are added together to get the total noise floor). This shows the within trial-pair variability, not the trial-by-trial variability shown in the first column. It allows you to see if the MEMR is distinguishable from the measurement noise. Even when the MEMR are distinguishable from noise, often the trial-by-trial variability means we still cannot be sure we're measuring a MEMR reliably. This estimate is directly related to the SFOAE noise level. If the noise is high then the MEMR is masked (and typically the QQ variability is high too). However, the measurement noise can be very low and the QQ variability still very high. Note that since instituting SFOAE stopping rules, the MEMR "noise" is usually acceptable and at around the criterion stopping rules of $-10 \mathrm{~dB}$ SPL.

- MEMR \& MOCR plot: The right plot compares the MEMR estimates to the unscaled (raw) MOCR estimates. It is only meaningful if there is a reasonable SFOAE at $1 \mathrm{kHz}$. It is an added bonus as we can see how the MOCR I/O function might be affected by the presence of the MEMR.

o The key to understanding whether the MEMR affects the MOCR measurement is to consider the relative size of the MEMR to the raw MOCR. For example, if the MOCR is large (e.g., 15 dB SPL), a small MEMR (e.g., $0 \mathrm{~dB}$ SPL) is unlikely to sway the results of the test. However, if both the MOCR and the MEMR are of similar magnitude, the MEMR could have more impact on the test results (depending on both phase and magnitude). It is difficult to quantify because it is not a linear relationship - the MEMR affects both the stimulus input and the measured response. As a starting position though we can consider orders of magnitude - is the MEMR $>>$ MOCR or $<<$ MOCR or nearly the same? ${ }^{4}$ And how does the MOCR change as the BBN level increases?

o It should be noted that MOCR estimates from single SFOAE test frequencies measured in the 1-1.5 kHz range are not the most reliable (Marshall et al., in press). Therefore, we need to be careful not to make too much out of the current MOCR results, as these are just opportunistic estimates at $1 \mathrm{kHz}$, which might not be optimal for that ear (however, it could be that some of the observed unreliability in this frequency range was due to MEMR effects).

- There are two main issues - how reliably can we measure the MEMR, and how big does it have to be before it affects the MOCR? In this technical report, our focus is on the first issue.

\section{$6 \quad$ Results for Method 2}

We reviewed the results of MEMR measurement series made in 143 ears from 76 subjects. In summary, we were unable to clearly determine an appropriate BBN level to use for MOCR in 49 (34\%) ears. In 94 ears (66\%), we could deduce that $60 \mathrm{~dB}$ SPL was a safe level to test (e.g., $60 \mathrm{~dB}$ SPL clearly showed no MEMR, or the MEMR was very small). Of those 94 ears, when tested at $75 \mathrm{~dB}$ SPL, 37 ears had no MEMR and 57 ears had a measurable MEMR reflex. Figure 5 shows an example, and the Appendix starting on page 26 shows commented examples for 19 other ears ranging from clearly distinct MEMR thresholds to noisy and unusable results.

For our purposes in the MOCR experiments that these data came from, a MEMR must be above $0 \mathrm{~dB}$ SPL (and detectable) to be of interest, even if it is reliably detectable at a lower level. This is partly because for many ears, the inherent variability is also around $0 \mathrm{~dB}$ SPL, or higher. Later on in the

\footnotetext{
${ }^{4}$ The $>>$ and $<<$ symbols mean very much greater than or very much less than (ie not just $>$ or $<$ than, but orders of magnitude in difference).
} 
experiment, we raised this arbitrary criterion to $5 \mathrm{~dB}$ SPL. These decisions were made prior to realizing that the relative magnitudes of MEMR and MOCR needed to be considered.

\section{$7 \quad$ Method 3: HearID+MOCR at the MOCR test frequency}

So far we have estimated the MEMR threshold by making a MOCR measurement at $1 \mathrm{kHz}$, and reanalyzing it to extract the MEMR magnitude. This method is more sensitive than clinical MEMR methods when inherent variability is low, but it is still insensitive for many ears because small MEMR are potentially masked with variability. It is also unclear how stable it is from day to day. In other words, we don't know how well this test predicts the effect of MEMR on a different day at possibly a different frequency.

In Method 1 and 2, we considered the MEMR at $1 \mathrm{kHz}$. However, for SFOAE-MOCR measurements, it is of more interest to know if there was a MEMR affecting the test frequency, rather than a MEMR elsewhere. It is also of interest to know if there is a MEMR evoked during the SFOAE-MOCR measurement, and not just the potential for one. It also seems rather obvious in hindsight that Method 2 Variation 1 can be applied without modification to any SFOAE measurement made using a contralateral stimulus, such as when measuring the MOCR. In our earlier experiments, we used the MEMR measurement to screen for ears that had a low MEMR threshold, and we were also measuring transientevoked otoacoustic emissions (TEOAEs), which are wideband, so it was necessary to use a more general MEMR test (at $1 \mathrm{kHz}$ ).

For SFOAE-MOCR, we chose the test frequencies individually for each ear to maximize the SFOAE SNR by choosing high amplitude regions, and to ensure we are not testing near a spontaneous otoacoustic emission (SOAE). Although we could apply Method 3 to any of our datasets, we evaluated it on some more recent data made with the MOCR module, because these datasets were easier to work with, and it was clearer when there were recalibrations (when calculating variability estimates, we needed to take care to not combine measurements made with different calibrations as they can affect phase).

In summary, Method 3 is essentially identical to Method 2 Variation 1 other than $f_{p}$ can vary.

\subsection{Subjects}

Fourteen adult subjects (sixteen ears, 9 females, 5 males, age 19-29 years) were enrolled. Subjects were from an experiment designed to establish the relationship among MOCR estimates made at nearby frequencies using an SFOAE test. Subjects had normal hearing, and no known auditory pathology. All testing was conducted at MIT. The experimental protocol was approved by that institute's internal review board and conducted in compliance with regulations and ethical guidelines on experimentation with human subjects. Subjects provided informed consent and were paid for their participation in the experiments.

Subjects were tested for MEMR thresholds using Method 2, but we did not use the results to exclude subjects. S386R, S400R, S402R did not get MEMR $1 \mathrm{kHz}$ screening tests (an oversight), and S411L had variable results; the rest did not show a significant reflex at $65 \mathrm{~dB}$ SPL.

\subsection{Equipment \& Stimuli}

Testing was done using HearID 5.0 with the custom-built MOCR module (v1.98). The MOCR module automated the MOCR testing for both SFOAE and TEOAE-based tests. The contralateral elicitor was identical to that used in Method 2 - digital noise delivered via the M-Audio Transit to an ER2 tubephone. 
Only one contralateral stimulus level was used - $60 \mathrm{~dB}$ SPL - the level used for the MOCR test.

Stopping rules were set to ensure there was a minimum of 4 averages and a maximum of 20 averages and 12 rejects. The test stopped early if SNR reached $18 \mathrm{~dB}$ and the noise level was lower than $-10 \mathrm{~dB}$ SPL. Noise rejection was set to $55 \mathrm{~dB}$ SPL.

The two conditions were " $1 \mathrm{kHz}$ " and "Best” frequencies. All subjects completed $1 \mathrm{kHz}$, but 2 subjects did not complete Best.

- $\quad$ For the $1 \mathrm{kHz}$ condition, eleven $f_{p}$ frequencies surrounding $1 \mathrm{kHz}$ were tested, and all subjects were tested at these frequencies: 902, 926, 949, 973, 996, 1020, 1043, 1066, 1090, 1113, 1137 $\mathrm{Hz}$. These frequencies may not be optimal for that ear. Instead they were chosen for comparison to an earlier study by Backus and Guinan (2007).

- For the "Best" frequencies, 11 frequencies were chosen individually for each ear, between 0.85 and $3 \mathrm{kHz}$ (92 possibilities in total), targeting frequencies with good SNR, low noise, and relatively flat parts of the spectrum. These frequencies may overlap with the $1 \mathrm{kHz}$ range, but were offset by one frequency bin so were unique.

\subsection{Procedure}

Testers collected six MOCR trial-pairs per test frequency, and sometimes collected up to 3 more if there was time available and the on-screen results showed some data had not met quality control criteria.

\subsection{Data cleaning and initial processing}

The SFOAEs making up a trial-pair needed to meet the following criteria. These criteria were controlled automatically by the MOCR module and tests flagged and potentially repeated in real-time.

- Noise level $\leq-10 \mathrm{~dB}$ SPL.

- Individual stimulus levels within $3 \mathrm{~dB}$ of target, and the difference from their respective targets between the two stimuli also within $3 \mathrm{~dB}$.

- $\quad$ SFOAE SNR at least $9 \mathrm{~dB}$ (although this did not affect the MEMR estimation).

- At least 4 averages taken.

A trial-pair needed to meet the following criteria to be included in the average for the series. These criteria were controlled automatically by the MOCR module, and tests flagged and potentially repeated in real-time if they did not meet criteria.

- $\quad$ Both Q and N trials must meet the above SFOAE criteria.

- The $\mathrm{N}$ trial must complete within 34 seconds, timed from the end of the Q trial.

Testers were asked to aim for at least 6 quality measurements (which were shown in real-time during data collection; however, sometimes there wasn't time to do additional testing, or it was apparent that the OAE level was too low to meet criteria). To be included in the analysis, there needed to be at least 5 trialpairs meeting the above quality-control criteria.

\subsection{Deriving MEMR from MOCR measurements}

Briefly recapping Method 2, the key to making MEMR estimates from MOCR measurements is to recognize that in an SFOAE measurement, there are two sections: one with probe-alone and one with probe+suppressor. For MEMR, we ignore the probe-alone section as that has the stimulus and SFOAE 
components mixed together. The probe+suppressor section has no SFOAE component because the $60 \mathrm{~dB}$ SPL suppressor suppresses it leaving only the stimulus (and noise) at the probe frequency (40 dB SPL). Now in the $\mathrm{N}$ trial, the contralateral elicitor could activate the MEMR. By comparing the stimulus in the two probe+suppressor sections (one from the $\mathrm{Q}$ trial and one from the $\mathrm{N}$ trial), we can take the vector difference and thereby estimate the MEMR magnitude in $\mathrm{dB}$ SPL.

By re-pairing the trials, we can also get an estimate of inherent variability by comparing adjacent Q trials where there was no contralateral MEMR elicitor.

\subsection{MEMR Analysis and screening criteria}

MEMR quality control criteria were essentially identical to the MOCR quality control criteria, other than an SFOAE SNR criterion was not applied (the SFOAE is suppressed so is irrelevant).

When the trials were reordered for MOCR and MEMR variability estimates, it was possible for the two trials in the pair to have different calibrations. This happened frequently as testers were allowed to recalibrate between QN trial pairs (all MOCR and MEMR trial-pairs used the same calibration within the QN pair - this was forced by the MOCR module). Such trials were flagged as not meeting quality control as the calibration can cause large phase shifts.

In the plots, all trials were plotted, but only the trials meeting quality control criteria were plotted as solid symbols (as opposed to open symbols), and contributed to the box and whisker plots, as shown in Appendix D.

\section{Results for Method 3}

In the main experiment, we found more MOCR variability around $1 \mathrm{kHz}$, compared to the Best frequencies, and surmised that this could be the MEMR, which has its biggest effects around $1 \mathrm{kHz}$. Previously we have shown with our SFOAE-MOCR based MEMR test that MEMR magnitude may be greater than the raw MOCR effect, thereby being a potential confound. We have also shown that the inherent variability in this MEMR test can also be at a level greater than the MEMR and the raw MOCR effect, making it impossible to conclude much about the role of the MEMR on MOCR measurements in many ears, because we can't separate its effects from variability. It's also unclear if this variability itself could affect MOCR measurements. ${ }^{5}$

The ultimate aim is to find a quantitative method to flag those trials affected by MEMR. As stepping stones to this aim, two graphical analysis iterations were done to get a feel for the data:

1. Each MOCR/MEMR trial series was plotted so the effects on individual measurements could be seen. Some examples are shown in the Appendix starting on page 36.

2. A MEMR, raw MOCR, and MOCR\% (which is the raw MOCR scaled by the SFOAE magnitude) summary for each ear and frequency, with trial-by-trial variability of the measurement and its inherent variability plotted as box and whisker plots. Frequency spacing of the box and whisker plots is in order but spaced to reflect the actual frequency spacing. This has the downside of making the labels indistinguishable in many cases. See the Appendix starting on page 42 for all 16 ears.

\footnotetext{
${ }^{5}$ MEMR variability (which could also be construed as stimulus level stability) is less likely to affect MOCR estimates because MOCR is derived from SFOAEs, and the SFOAE is derived from interleaved probe-alone and probe+suppressor measurements, that occur very close together in time. In comparison the variability seen in the MEMR measurements is from fluctuations in stimulus level from $Q$ trial to $Q$ trial.
} 


\subsection{Summarizing the MEMR analyses}

A summary of the MEMR results using Method 3 for each subject is shown in the Appendix on page 42. There is no strong indication that a high MEMR variability is associated with poor MOCR results. Visual inspection indicates that there were no obvious MEMR for any subject at any frequency (i.e., a MEMR magnitude $>>0 \mathrm{~dB}$ SPL, such as shown in Figure 3), but variability was often high and could have masked a small MEMR. As shown in Table 1, there are some cases where a masked MEMR response could be the same or higher order of magnitude as the raw MOCR and thereby interfere with the MOCR, but we cannot say for certain. It would be interesting to run this experiment with higher contralateral BBN levels to see how MEMR and MOCR varied with frequency.

It should be noted that this study was not designed to establish how measurable the MOCR might be or to establish the stability of the MOCR across frequency. Many sub-optimal frequencies were tested for comparison to an earlier study that used fixed frequencies. So the fact that the MOCR was not measurable or was variable in some ears should not be taken as a sign that the test is poor - it was to be expected. Here we are merely taking advantage of this existing dataset merely to see how the MEMR may be derived from such measurements, and to see if it relates to MOCR variability. 
Table 1. Summary of the key patterns for the 16 ears. MEMR present (yes, no, or ? for too variable); MEMR variability, raw MOCR, and MOCR\% were rated as being low, medium, or high magnitudes. We particularly wanted to note if high MEMR variability was related to MOCR in case the high variability was masking an MEMR. See Appendix E for the associated box plots.

\begin{tabular}{|c|c|c|c|c|c|}
\hline Ear & $\begin{array}{c}\text { MEMR } \\
\text { present? }\end{array}$ & $\begin{array}{l}\text { MEMR } \\
\text { variability* }\end{array}$ & $\begin{array}{l}\text { Raw } \\
\text { MOCR }\end{array}$ & MOCR\% & Comments \\
\hline 338L & $?$ & High & $\begin{array}{l}\text { Low - } \\
\text { Mod }\end{array}$ & Low & $\begin{array}{l}\text { MOCR undetectable and missing data; } \\
\text { could be affected by MEMR }\end{array}$ \\
\hline 353L & No & Mod & High & Mod & $\begin{array}{l}\text { Only " } 1 \mathrm{kHz} \text { " frequency group tested; } \\
\text { MOCR detectable; any MEMR activity } \\
\text { unlikely to have affected MOCR }\end{array}$ \\
\hline 368L & No or? & Low - High & $\begin{array}{l}\text { Low - } \\
\text { Mod }\end{array}$ & $\begin{array}{l}\text { Low - } \\
\text { Mod }\end{array}$ & $\begin{array}{l}\text { MOCR detectable but some missing data; } \\
\text { strength varies across frequency; MEMR } \\
\text { activity probably didn't affect MOCR }\end{array}$ \\
\hline 370R & No or? & Low - High & Low & Low & $\begin{array}{l}\text { MOCR undetectable and missing data; } \\
\text { MEMR activity unlikely to have affected } \\
\text { MOCR }\end{array}$ \\
\hline $371 L$ & No & Low - Mod & Low & Low & $\begin{array}{l}\text { MOCR undetectable; MEMR unlikely to } \\
\text { have affected MOCR }\end{array}$ \\
\hline $371 R$ & No or? & Mod & $\begin{array}{l}\text { Low - } \\
\text { Mod }\end{array}$ & $\begin{array}{l}\text { Low - } \\
\text { Mod }\end{array}$ & $\begin{array}{c}\text { MOCR\% at max around } 1-1.1 \mathrm{kHz} ; \text { MEMR } \\
\text { unlikely to have affected MOCR }\end{array}$ \\
\hline 372R & No or? & Mod - High & High & $\begin{array}{l}\text { Mod - } \\
\text { High }\end{array}$ & $\begin{array}{l}\text { MOCR detectable except at SFOAE null; } \\
\text { strength varies across frequency; not } \\
\text { likely to be affected by MEMR activity }\end{array}$ \\
\hline 395L & No & Low & $\begin{array}{l}\text { Mod - } \\
\text { High }\end{array}$ & Mod & $\begin{array}{c}\text { MOCR detectable and constant, but } \\
\text { variable below } 1 \mathrm{kHz} \text {; unaffected by MEMR } \\
\text { activity }\end{array}$ \\
\hline 396L & No & Low & $\begin{array}{l}\text { Low - } \\
\text { Mod }\end{array}$ & Low & $\begin{array}{l}\text { MOCR undetectable; unlikely to be } \\
\text { affected by MEMR activity }\end{array}$ \\
\hline 398R & No & Low - Mod & $\begin{array}{l}\text { Low - } \\
\text { Mod }\end{array}$ & Low & $\begin{array}{l}\text { MOCR undetectable; unlikely to be } \\
\text { affected by MEMR activity }\end{array}$ \\
\hline 399L & No or? & Low - Mod & High & High & $\begin{array}{l}\text { MOCR low below } 1 \mathrm{kHz} \text { and high above; } \\
\text { unlikely to be affected by MEMR activity }\end{array}$ \\
\hline 400R & $?$ & High & Low & Low & $\begin{array}{l}\text { MOCR undetectable; could be affected by } \\
\text { MEMR activity }\end{array}$ \\
\hline 401R & $?$ & Mod & Mod & $\begin{array}{l}\text { Mod - } \\
\text { High }\end{array}$ & $\begin{array}{l}\text { MOCR detectable but variable; strength } \\
\text { varies across frequency; could be affected } \\
\text { by MEMR activity }\end{array}$ \\
\hline 402L & No or? & Mod - High & Mod & $\begin{array}{l}\text { Low - } \\
\text { Mod }\end{array}$ & $\begin{array}{l}\text { MOCR detectable; could be affected by } \\
\text { MEMR }\end{array}$ \\
\hline 402R & No or? & Mod - High & Mod & $\begin{array}{l}\text { Low - } \\
\text { Mod }\end{array}$ & $\begin{array}{l}\text { MOCR detectable; strength varies across } \\
\text { frequency; could be affected by MEMR } \\
\text { activity, but not likely }\end{array}$ \\
\hline 411L & No or? & High & Mod & Mod & $\begin{array}{l}\text { MOCR undetectable due to variability; } \\
\text { MEMR activity could have affected } \\
\text { MOCR.marshall }\end{array}$ \\
\hline
\end{tabular}

* No ear showed a MEMR. This column indicates if the MEMR inherent variability was low, moderate, or high. If it is high, a true but small MEMR might be masked. 


\section{Discussion}

This report covers three related methods for estimating the MEMR threshold and magnitude using OAE equipment. Although our original motivation in measuring the MEMR was to establish its effect on the MOCR, this report is primarily about how to make the MEMR measurement and how effectively this can be done. Analysis and discussion on the relationship between the MEMR and MOCR is outside the scope of this document, although there is frequent mention of it in the results and appendices for interested readers, and for giving the wider context.

Our experience is that MEMR is reliably measurable at levels lower than detectable with clinical tympanometers in some ears, but not others.

- It is rare that we get a clean data run across all BBN levels, so we are left to intuit whether the results in front of us show a MEMR effect.

- Often, the MEMR is clearly separable from the derived MEMR noise floor, but is not separable from the inherent trial-by-trial variability. We note that our variability is an over-estimate because it compares trials twice as far apart in time as the trials that contribute to the MEMR measurement. An alternative approach is to make additional measurements to allow a more comparable estimate to be made: e.g., using a QQNQQNQQN sequence rather than QNQNQN..., however this increases the number of trials needed by a third.

- There appears to be a slow variation to the pressure in some ears that make it intrinsically difficult to reliably get a MEMR result. The nature of our three methods where the pressure is considered in the frequency domain does not allow us to view these slowly-varying time effects like the method used by Lilaonitkul and Guinan (2009a, 2009b). However, their procedure is not easily implementable on standard audiological equipment.

- It is not clear how large a MEMR must be before it makes an obvious and meaningful impact on the derived MOCR. We started with $0 \mathrm{~dB}$ SPL for the MEMR magnitude (providing it was clearly separable from noise and variability), but raised it to $5 \mathrm{~dB}$ SPL because too many subjects were not getting clear results and excluded from the experiments. For the specific case of assessing MEMR threshold for MOCR measurements, the criterion for MEMR magnitude should be considered relative to the MOCR magnitude. If the two reflexes affect similar frequency ranges and have similar magnitudes, thus they are potentially confounds for one another. This has implications for a clinical test where there is not necessarily enough time to make all the measurements needed for this detailed assessment.

- For method 2, we have a small amount of data where the subject had a strong SFOAE at $1 \mathrm{kHz}$ allowing for simultaneous MOCR and MEMR estimates. In general, SFOAE-MOCR at $1 \mathrm{kHz}$ is not particularly reliable (Backus \& Guinan, 2007; Marshall et al., in press). Is $1 \mathrm{kHz}$ just a bad frequency to choose? We can't go much lower as the noise levels worsen, but we can't go much higher in frequency if we want to measure the full impact of the MEMR, which is primarily a low-frequency effect.

- Method 3 provides a way to assess the impact of the MEMR at the time of the MOCR measurement, at the test frequency of interest without needing additional measurements. However, if MEMR threshold is needed for subject screening, Method 2 might be preferable.

- The method chosen depends too on the OAE type used for the MOCR method. If SFOAEs using tonal stimuli are used, then we can use method 3 and derive MEMR from the actual measurement. We are currently working on a similar method for broadband TEOAE stimuli. Henin et al. (2014) have shown for swept-OAEs how MEMR activity can be derived by comparing up-sweeps to downsweeps. 


\subsection{Source of variability}

In a separate, informal exercise, colleagues at MIT investigated potential sources of variability in their ears (both of which showed repeatedly high intrinsic variability), including:

- Attention by the subject to the stimulus. Attention was controlled by asking the subject to count the number of probe/suppressor cycles.

- Increasing the time between QN trial-pairs to allow the MEMR to return to baseline (although this increases the duration between pairs used to estimate trial-by-trial variability so can increase variability).

- Increasing the number of trial-pairs to 20 to see if more stable, potentially lower, estimates of variability were obtained.

- Looking for changes in wideband acoustic immittance at $1 \mathrm{kHz}$ (this method was more variable). This method showed a lot of measurement drift, which indicates something about the middle ear is slowly varying in time (possibly middle-ear muscle tonus fluctuations, middle-ear pressure fluctuations, or environmental factors such as heat or humidity).

None were able to account for, explain, or decrease the variability seen.

\subsection{Limitations}

By considering inherent variability, we reveal how difficult it can be to isolate very small MEMR effects reliably. This is certainly an issue for all techniques looking at small MEMR, but usually is not considered. Historically and clinically, the large MEMR effect is what is of interest, but it's the small MEMR effects that can confound MOCR, and other similar measurements, because they can be the same order of magnitude.

For laboratory research on the MOCR, we want to be able to test the MOCR independently of the MEMR. For field research, we ideally want to at least be able to categorize those subjects with "pure" MOCR measurements, and those with combined MOCR/MEMR measurements to know what is predictive and how well it works. For ultimate implementation, it may not matter that there is some MEM activity mixed in with the MOCR measurement (Luebke et al., 2014). If this is the case, we may need to determine what level of MEMR interference is acceptable without lowering the prediction. These are important questions to ask because although both the MEMR and the MOCR are potentially protective, they do not behave identically. For example, the MEMR response is history dependent (i.e., its activation depends on preceding sounds), and the response decays over time (Gerhardt, Melnick, \& Ferraro, 1980; Rodriguez \& Gerhardt, 1988); whereas the MOCR is stable and even may increase in strength over many minutes (van Zyl, Swanepoel, \& Hall, 2009).

\section{Conclusions}

In the current report, examples for three methods to measure the MEMR using OAE equipment show a variety of test outcomes. When they work, they work beautifully, but we estimate in only around two thirds of ears is a threshold determinable. In summary, the benefits to these methods are that the MEMR test is sensitive, can be derived from the MOCR test, and can be made relatively simply using commercially-available OAE systems. However, clinical implementations and applications of these methods are not viable due to the high inherent trial-to-trial variability seen in many ears. Alternative, sensitive, MEMR tests should continue to be pursued, especially those that can track the change in stimulus level faster.

It is important to be able to detect MEM activity while making MOCR measurements with OAEs. Each OAE type presents its own challenges. Of the various efforts that have been made Goodman et al. 
(2013) and Xu et al. (2014) for TEOAEs; Henin et al. (2014) for swept-OAEs; Lilaonitkul and Guinan (2009a, 2009b) and us (this report) for SFOAEs; and Luebke et al. (2014) for DPOAE adaptation), none are ready for clinical implementation.

\section{References}

Backus, B. C., \& Guinan, J. J. (2007). Measurement of the distribution of medial olivocochlear acoustic reflex strengths across normal-hearing individuals via otoacoustic emissions. $J$ Assoc Res Otolaryngol, 8(4), 484-496.

Buki, B., Wit, H. P., \& Avan, P. (2000). Olivocochlear efferent vs. middle-ear contributions to the alteration of otoacoustic emissions by contralateral noise. Brain Res, 852(1), 140-150.

Feeney, M. P., \& Keefe, D. H. (2001). Estimating the acoustic reflex threshold from wideband measures of reflectance, admittance, and power. Ear Hear, 22(4), 316-332.

Gerhardt, K. J., Melnick, W., \& Ferraro, J. A. (1980). Acoustic reflex decay in chinchillas during a longterm exposure to noise. Ear Hear, 1(1), 33-37.

Goodman, S. S., \& Keefe, D. H. (2006). Simultaneous measurement of noise-activated middle-ear muscle reflex and stimulus frequency otoacoustic emissions. J Assoc Res Otolaryngol, 7(2), 125-139.

Goodman, S. S., Mertes, I. B., Lewis, J. D., \& Weissbeck, D. K. (2013). Medial olivocochlear-induced transient-evoked otoacoustic emission amplitude shifts in individual subjects. J Assoc Res Otolaryngol, 14(6), 829-842. doi: 10.1007/s10162-013-0409-9

Guinan, J. J., Jr., Backus, B. C., Lilaonitkul, W., \& Aharonson, V. (2003). Medial olivocochlear efferent reflex in humans: otoacoustic emission (OAE) measurement issues and the advantages of stimulus frequency OAEs. $J$ Assoc Res Otolaryngol, 4(4), 521-540.

Henin, S., Long, G. R., \& Thompson, S. (2014). Wideband detection of middle ear muscle activation using swept-tone distortion product otoacoustic emissions. Journal of the Acoustical Society of America, 136(1), 272. doi: 10.1121/1.4883361

Lilaonitkul, W., \& Guinan, J. J., Jr. (2009a). Human medial olivocochlear reflex: effects as functions of contralateral, ipsilateral, and bilateral elicitor bandwidths. Journal of the Association for Research in Otolaryngology, 10(3), 459-470. doi: 10.1007/s10162-009-0163-1

Lilaonitkul, W., \& Guinan, J. J., Jr. (2009b). Reflex control of the human inner ear: a half-octave offset in medial efferent feedback that is consistent with an efferent role in the control of masking. Journal of Neurophysiology, 101(3), 1394-1406. doi: 10.1152/jn.90925.2008

Luebke, A. E., Stagner, B. B., Martin, G. K., \& Lonsbury-Martin, B. L. (2014). Adaptation of distortion product otoacoustic emissions predicts susceptibility to acoustic over-exposure in alert rabbitsa). The Journal of the Acoustical Society of America, 135(4), 1941-1949. doi: doi:http://dx.doi.org/10.1121/1.4868389

Marshall, L., Lapsley Miller, J. A., Guinan Jr., J. J., Shera, C., Reed, C., Delhorne, L., \& Boege, P. (in press). Otoacoustic-emission-based medial-olivocochlear reflex assays for humans. Journal of the Acoustical Society of America.

Rodriguez, G. P., \& Gerhardt, K. J. (1988). Adaptation properties of the acoustic reflex in response to continuous-, intermittent- and industrial-noise stimulation. Audiology, 27(6), 344-355.

Shera, C. A., \& Guinan, J. J., Jr. (1999). Evoked otoacoustic emissions arise by two fundamentally different mechanisms: a taxonomy for mammalian OAEs. Journal of the Acoustical Society of America, 105(2 Pt 1), 782-798.

van Zyl, A., Swanepoel, D., \& Hall, J. W., 3rd. (2009). Effect of prolonged contralateral acoustic stimulation on transient evoked otoacoustic emissions. Hear Res, 254(1-2), 77-81. doi: S03785955(09)00098-7 [pii] 
10.1016/j.heares.2009.04.013

Xu, Y., Cheatham, M. A., \& Siegel, J. (2014). Separating Medial Olivocochlear from Acoustic Reflex Effects on Transient Evoked Otoacoustic Emissions in Unanesthetized Mice. Paper presented at the Mechanics of Hearing 12th International Workshop, Cape Sound, Greece.

Zhao, W., \& Dhar, S. (2010). The Effect of Contralateral Acoustic Stimulation on Spontaneous Otoacoustic Emissions. J Assoc Res Otolaryngol, 11, 53-67. doi: 10.1007/s10162-009-0189-4 


\section{Appendix A: List of Acronyms}

- BBN: broadband noise

- dB SPL: sound pressure level in decibels

- DP or DPOAE: distortion-product OAE

- fp: probe frequency

- fs: suppressor frequency

- $\quad$ f1: first primary frequency

- $\quad \mathrm{f} 2$ : second primary frequency

- I/O: input/output

- IQR: interquartile range

- Lp: stimulus level at fp

- MEMR: middle-ear muscle reflex

- MOCR: medial olivocochlear reflex

- OAE: otoacoustic emission

- Pp: probe-alone pressure

- Pps: probe+suppressor pressure

- Q: quiet trial with no contralateral noise

- $\quad \mathrm{N}$ : noise trial with contralateral noise

- QN: quiet and noise trials forming a trial-pair combination

- QQ: two quiet trials forming a trial-pair combination

- SF or SFOAE: stimulus frequency OAE

- SNR: signal-to-noise ratio

- TEOAE: transient-evoked OAE 


\section{Appendix B: Method 1 examples of MEMR magnitude}

See MEMR Magnitude section on page 5 for details on the plots.

Subject 106 has no MEMR to the $50 \mathrm{~dB}$ SPL BBN, and low variability:
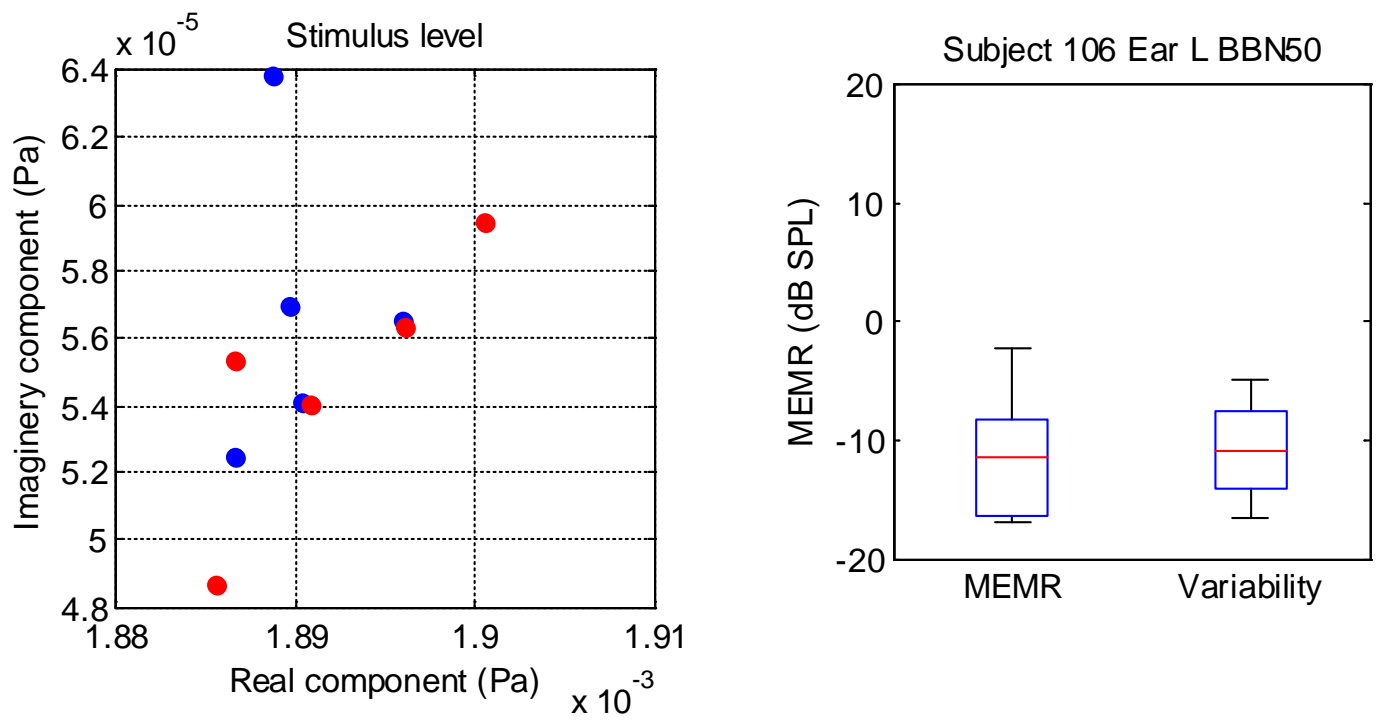

Subject 107 has a tiny MEMR to $60 \mathrm{~dB}$ SPL BBN, which is distinguishable from the variability, but it is probably too small to be of concern:
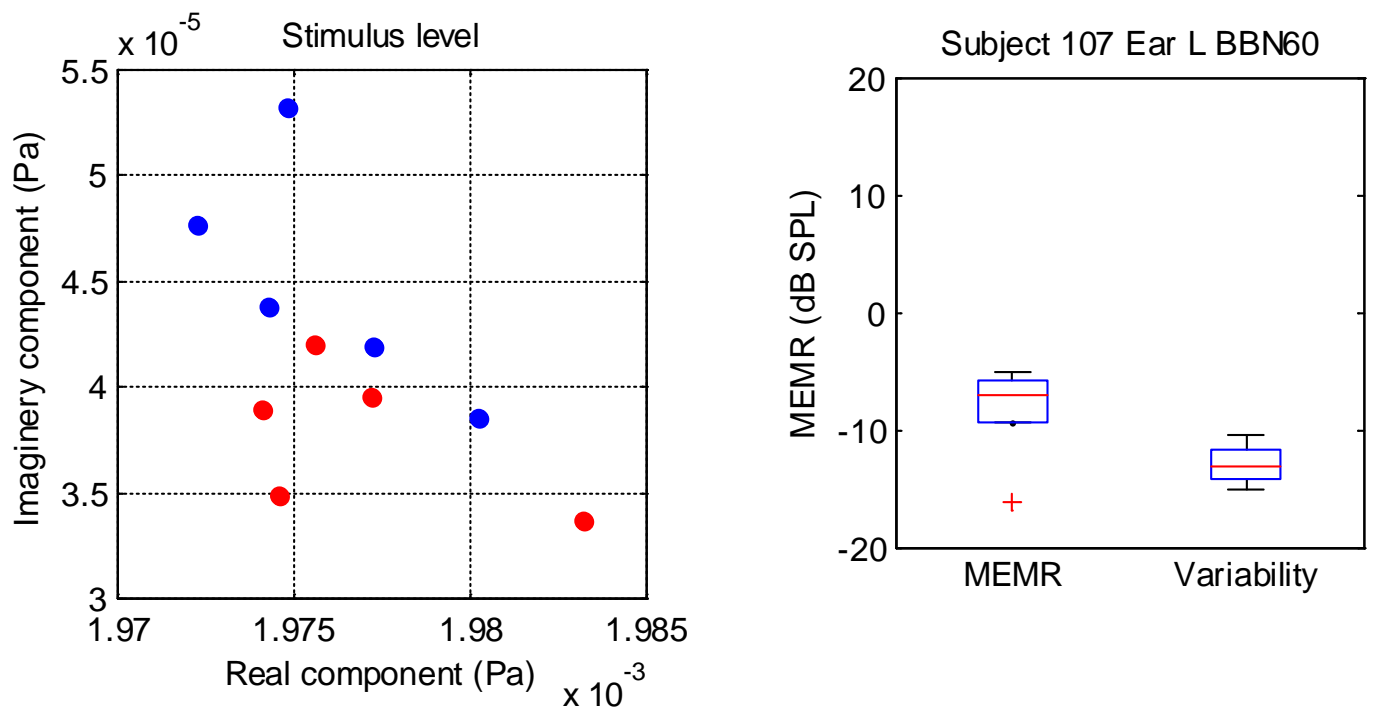
In this example with Subject 107, a TEOAE chirp stimulus at $44 \mathrm{~dB}$ SPL is used as the contralateral elicitor instead of the BBN. There is no MEMR, but variability is relatively high due to what looks like an ear probe that is slowly moving in the ear canal.

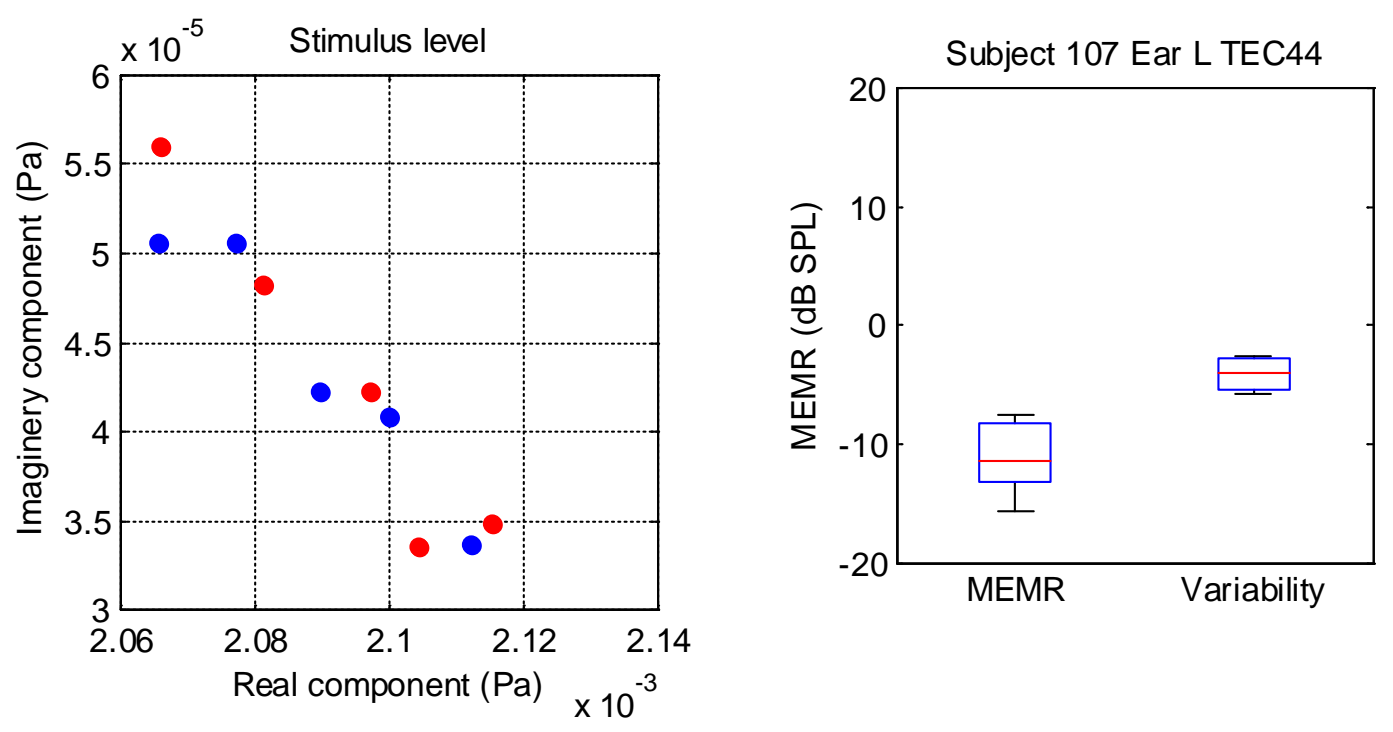

For Subject 107, a small MEMR partially overlaps with variability in the measurements.
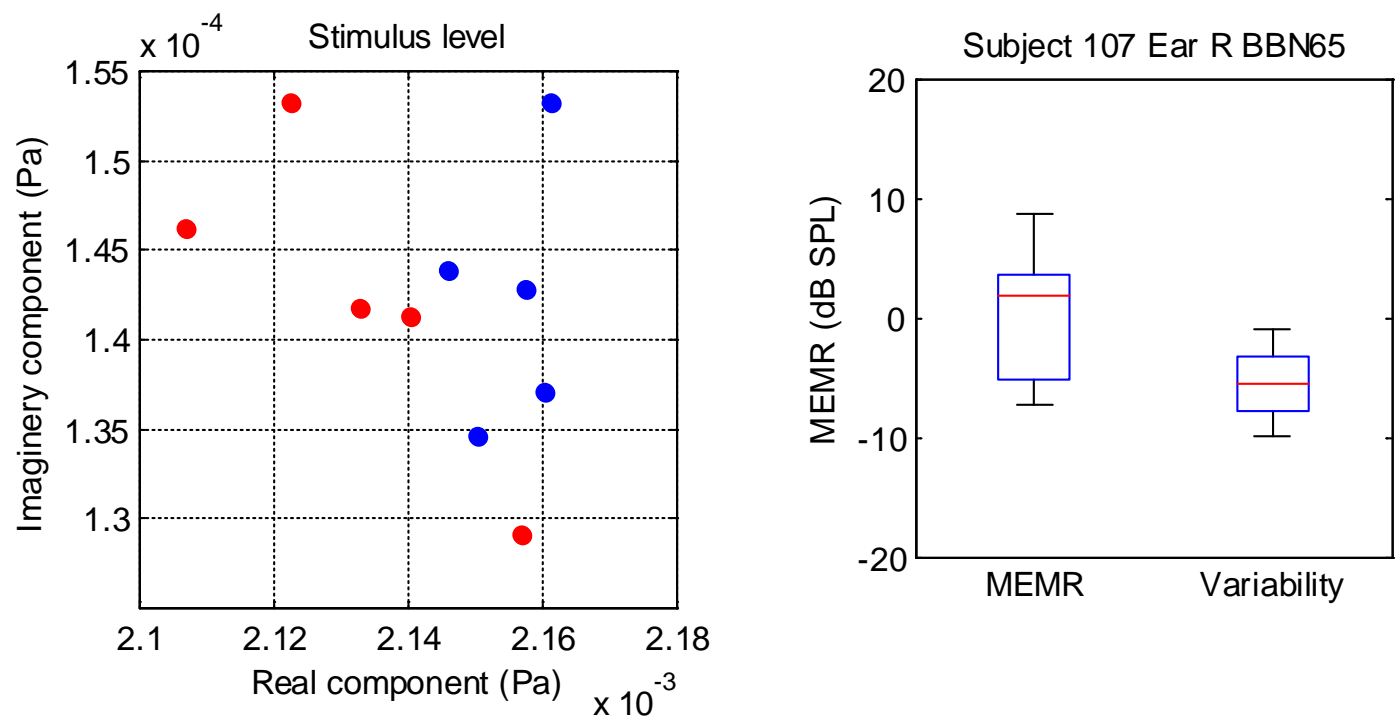
For subject 110, the large but variable MEMR to the $60 \mathrm{~dB}$ SPL BBN is indistinguishable from the variability - the MEMR may be a false alarm and just be due to trial-to-trial variability.
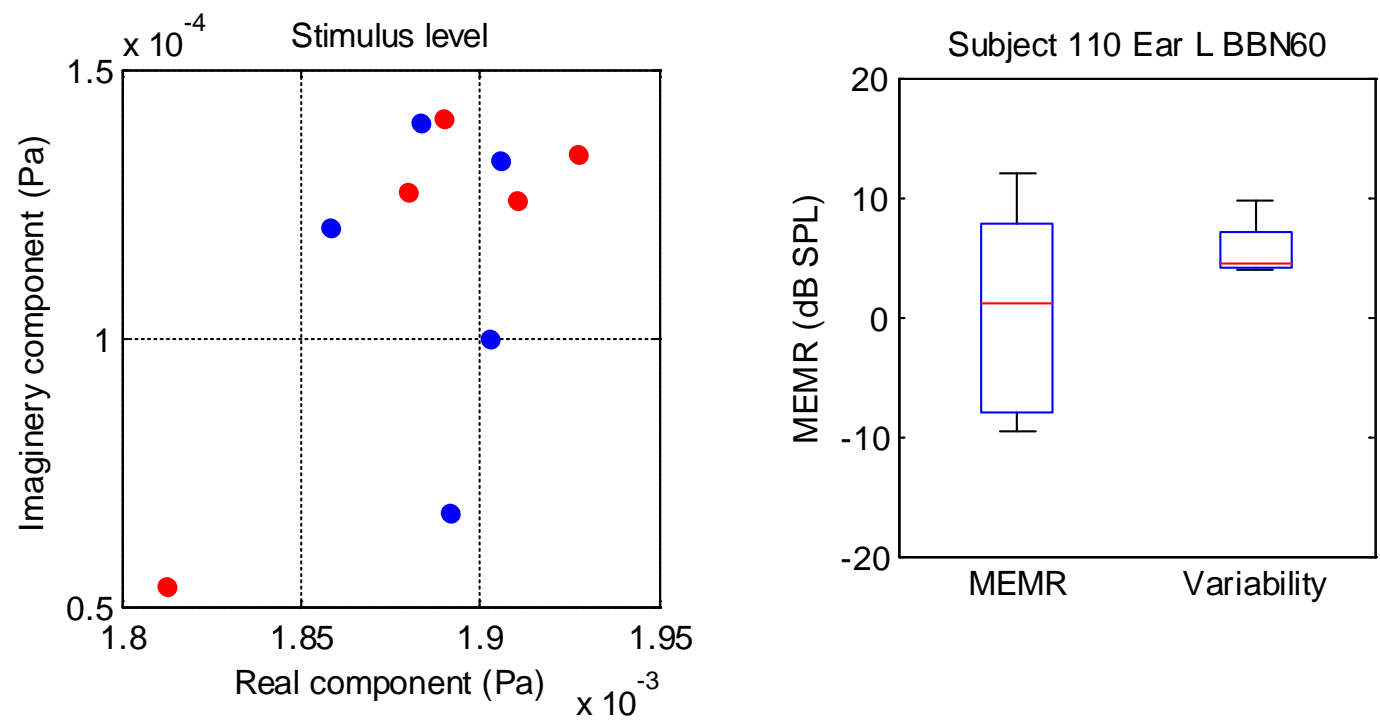

Subject 126 shows a large MEMR to a $65 \mathrm{~dB}$ SPL BBN and has low variability (also shown in Figure $3)$. The MEMR is clearly detectable.
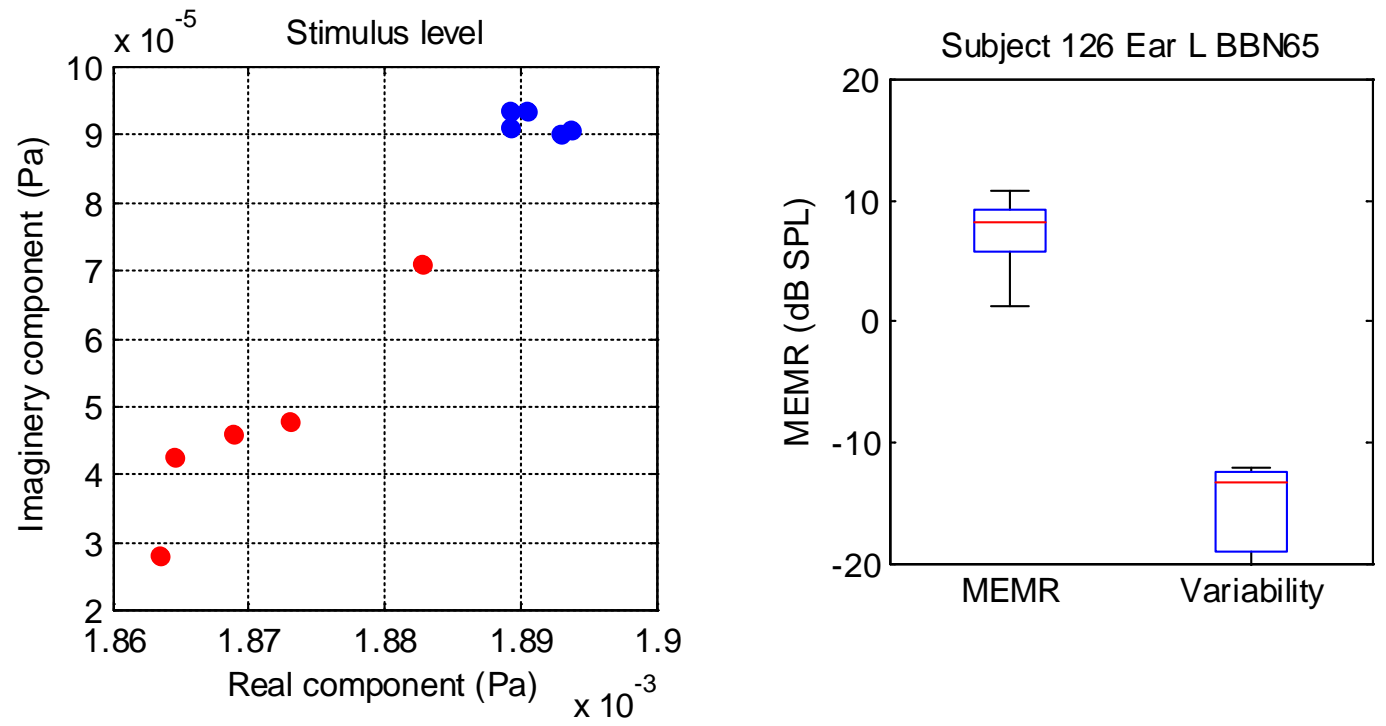


\section{Appendix C: Method 2 examples of MEMR magnitude and threshold}

\subsection{MEMR with clearly identifiable MEMR threshold}

\section{Subject ID 215, L Ear}
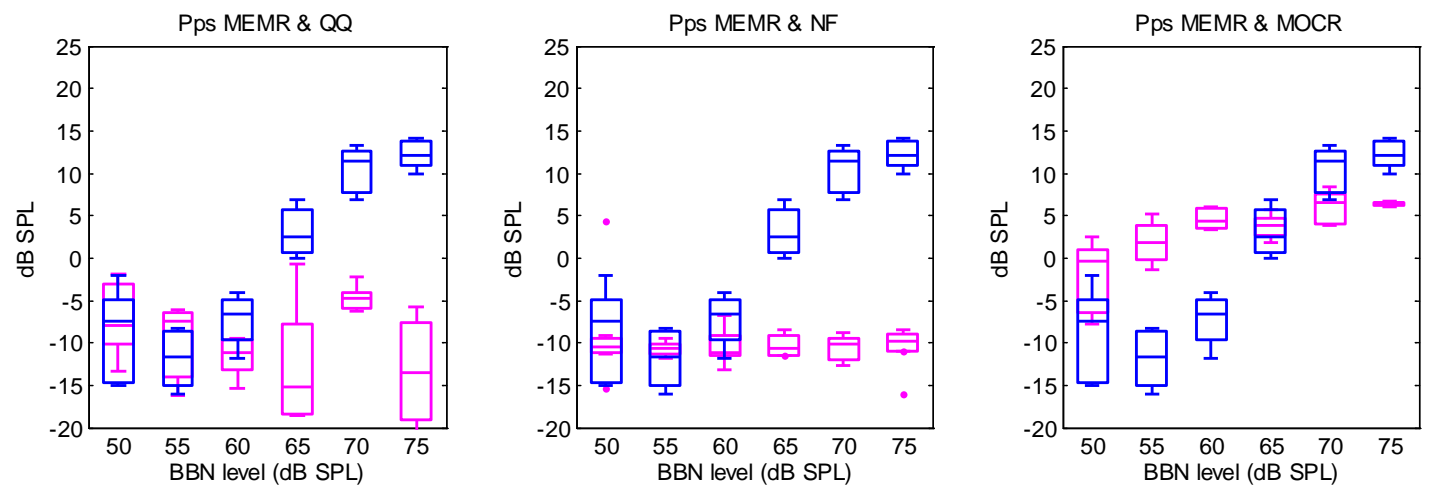

For 215L, a MEMR (blue) is present at $65 \mathrm{~dB}$ SPL and above, and is clearly distinguishable from variability (pink, left) and MEMR noise (pink, middle). The MOCR (pink, right) initially increases, presumably because the BBN level is not sufficient to fully engage the reflex, then plateaus at $60 \mathrm{~dB}$ SPL and doesn't change markedly at higher levels despite the presence of a MEMR of the same or larger magnitude from $65 \mathrm{~dB}$ and above. For levels $<65 \mathrm{~dB}$ SPL, the MEMR is not distinguishable from the (low) noise and variability and is $<<$ MOCR. It is unlikely that the MEMR affected the MOCR at $\mathrm{BBN}=60 \mathrm{~dB}$ SPL.

\section{Subject ID 242, L Ear}
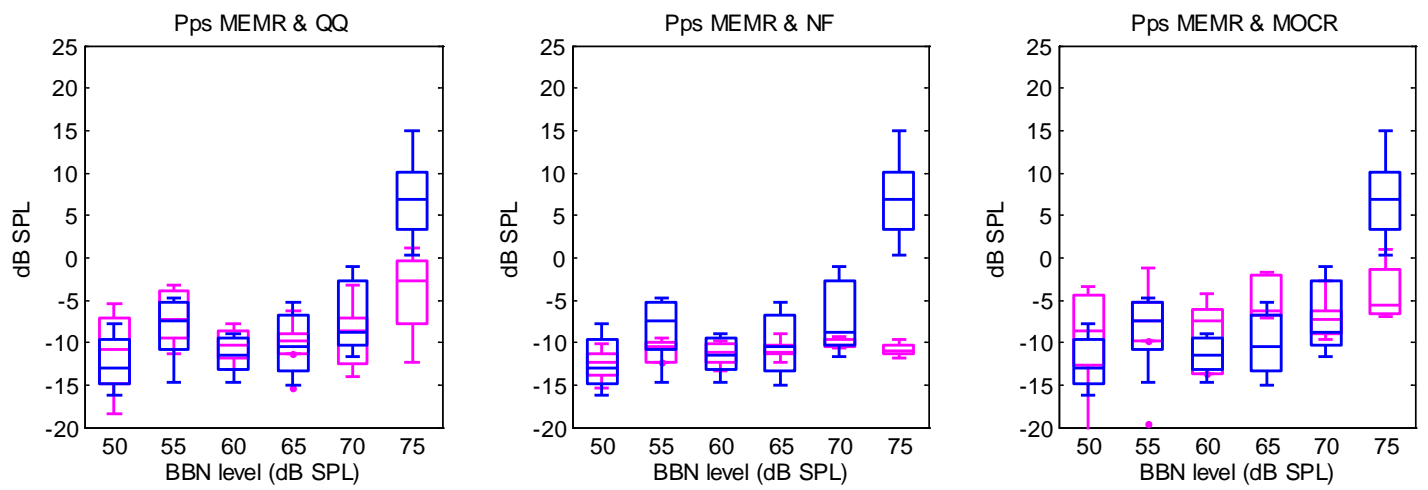

For Subject 242L, the MEMR threshold is clearly at $75 \mathrm{~dB}$ SPL and both variability and noise is low. A MOCR was not present at $1 \mathrm{kHz}$. 


\section{Subject ID 235, L Ear}
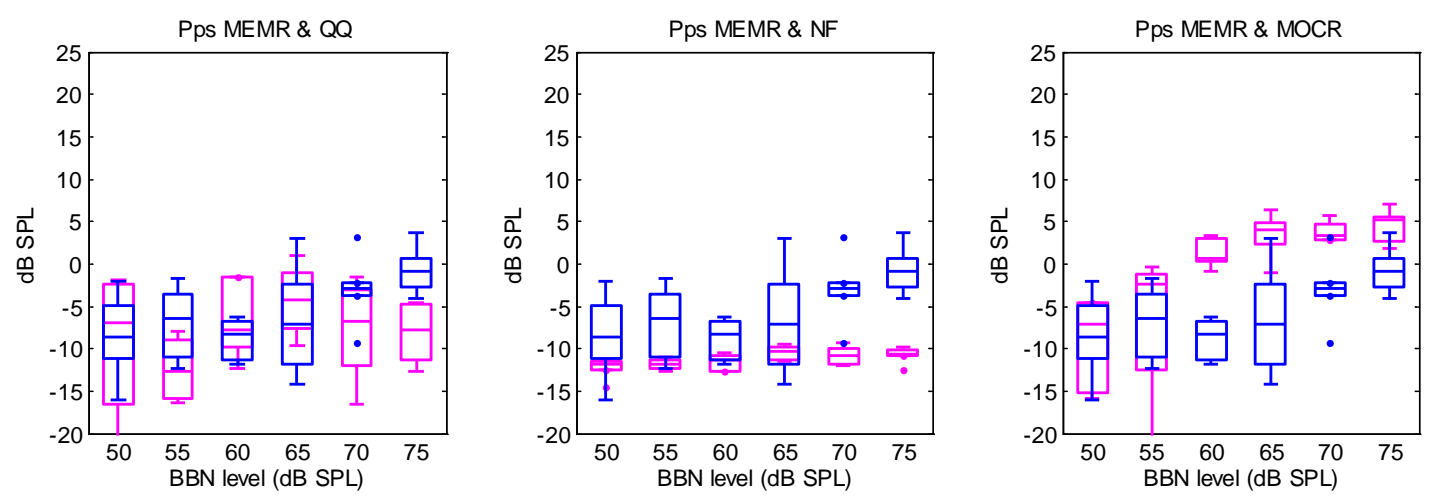

For Subject 235L, there may be a tiny reflex at 75 but it hasn't affected the MOCR (MEMR $<$ MOCR).

\section{Subject ID 308, L Ear}
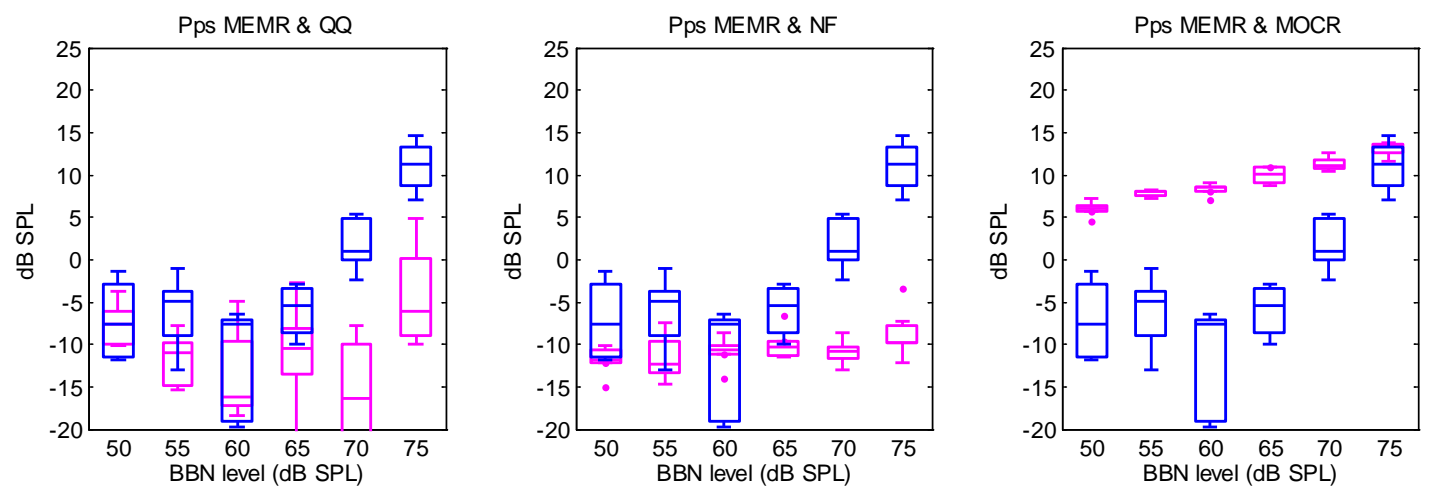

Subject 308 has a MEMR threshold around 70-75 dB SPL and a large MOCR. The MEMR $<$ MOCR at $70 \mathrm{~dB}$ SPL and equivalent at $75 \mathrm{~dB}$ SPL. The MOCR I/O function is increasing but at least for levels $<75 \mathrm{~dB}$ SPL, it is unlikely to be due to MEMR effects. 


\section{Subject ID 299, L Ear}
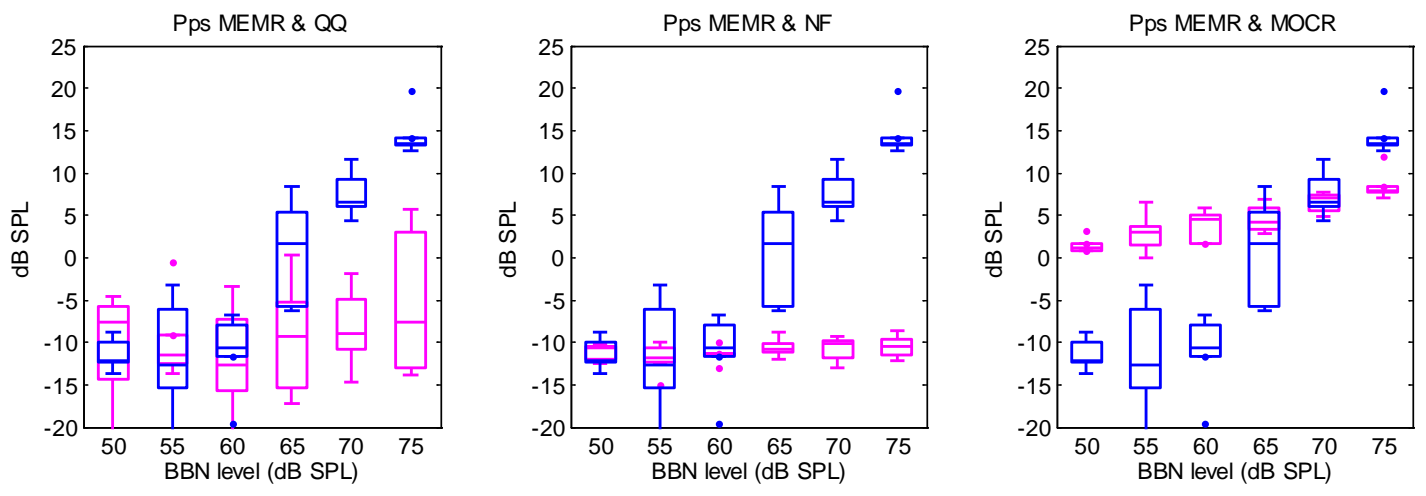

Subject 299L provides another example where MEMR is detectable at $65 \mathrm{~dB}$ SPL, although it is quite variable with the reflex engaging on some trials but not others. The MOCR is also measurable at $1 \mathrm{kHz}$, and plateaus around 55-65 dB SPL, but increases at 70-75 dB SPL possibly due to MEMR which is >= MOCR. The small MEMR at $65 \mathrm{~dB}$ SPL doesn't appear to affect the MOCR because the MOCR level is similar from 55-65 dB SPL.

\subsection{MEMR absent at all levels}

\section{Subject ID 251, L Ear}
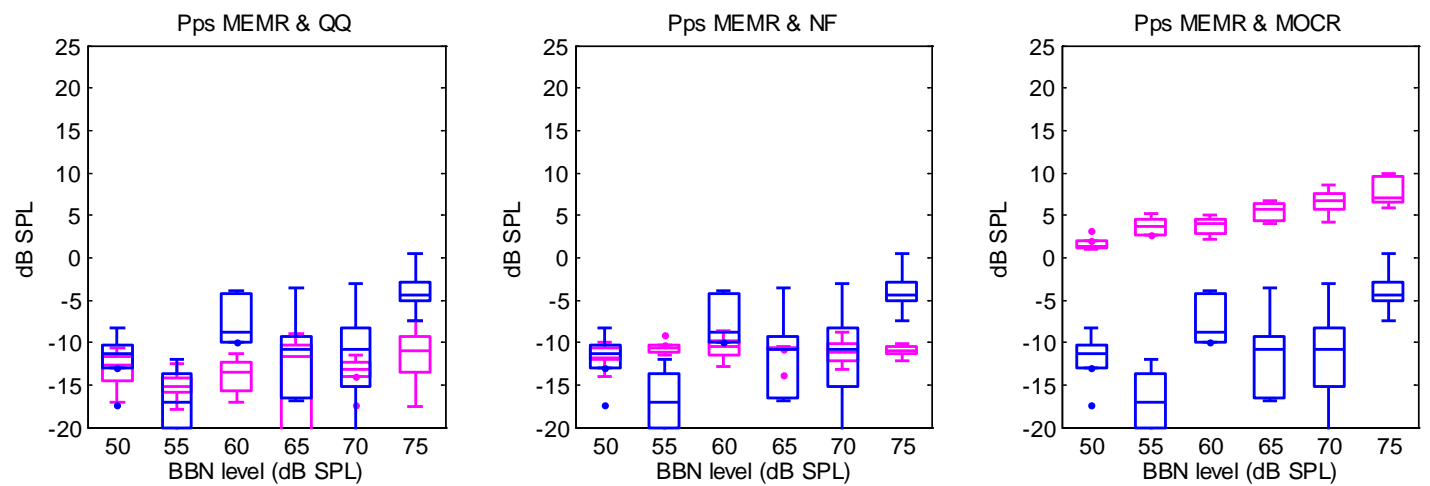

For Subject 251L, there may be a small MEMR reflex at $75 \mathrm{~dB}$ SPL, but it is tiny and $<<$ the MOCR. MOCR is measurable and increases with BBN level. The increase in MOCR at higher levels is unlikely to be due to a MEMR. 


\section{Subject ID 228, L Ear}
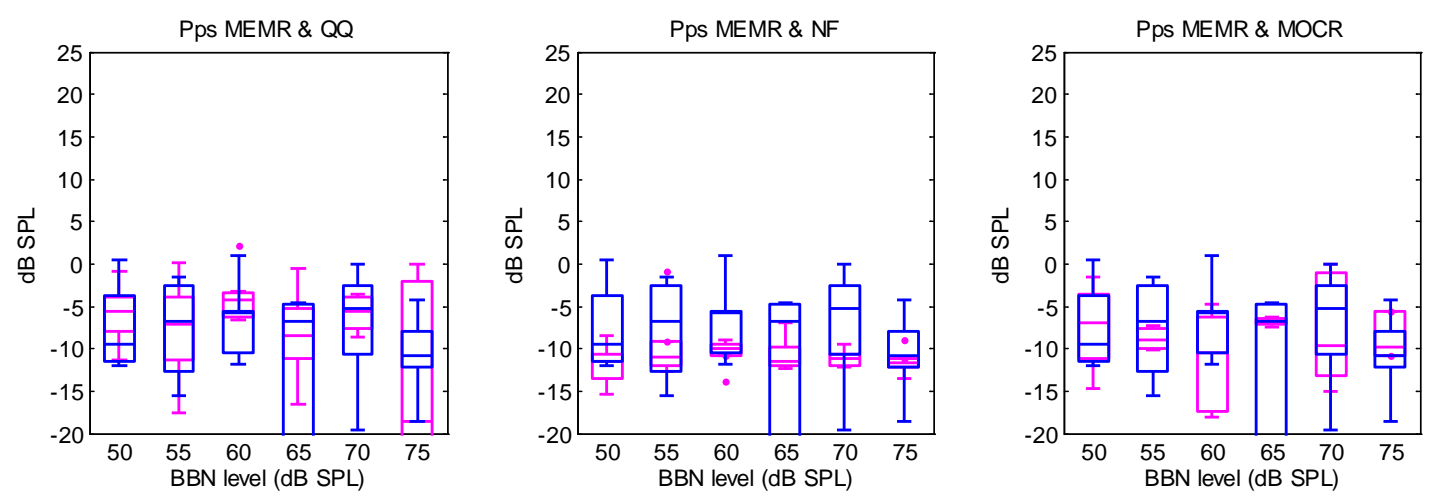

For Subject 228L, there is no MEMR present at any level tested. Variability is slightly higher than previous examples, but the results are clear - especially at $75 \mathrm{~dB}$ SPL. There is also no measurable MOCR.

\subsection{MEMR appears to be present, but is not detectable from variability}

In this scenario, there appears to be a MEMR (and there may even be the expected sigmoidal I/O function), but the MEMR magnitude is not reliably detectable from the trial-to-trial variability (pink, left). Note how there is no difference in the MEMR noise level (middle plot) to the previous "perfect" datasets - the variability is not directly related to noise in the individual OAE measurement.

\section{Subject ID 249, R Ear}
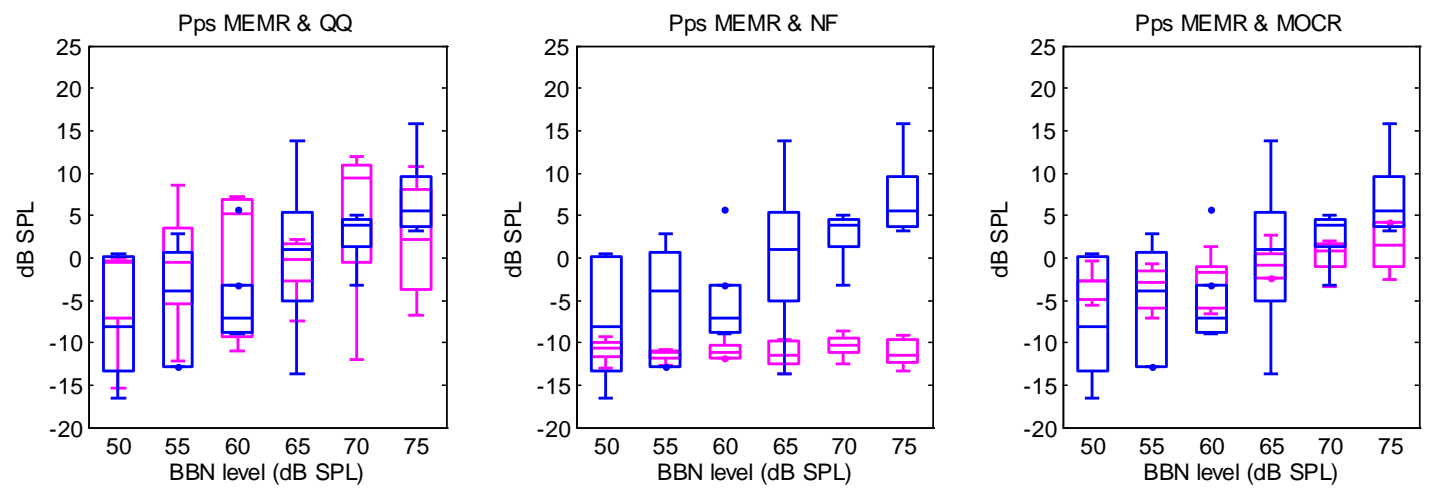

For Subject 249R, going by the middle plot, it looks like there is a MEMR reflex. However, it is not detectable from variability (left plot), and the MEMR itself is quite variable. Thus it is only safe to conclude that there is probably no reflex at $50 \mathrm{~dB}$ SPL. There could be a small MEMR at 55 or $60 \mathrm{~dB}$ SPL masked by variability. The magnitude of the MOCR is small and increasing and equal in magnitude to the MEMR. It is possible that the MOCR is affected by the MEMR. The subject was excluded from the MOCR experiment. 


\subsection{MEMR present at higher levels, ambiguous at lower Subject ID 287, L Ear}
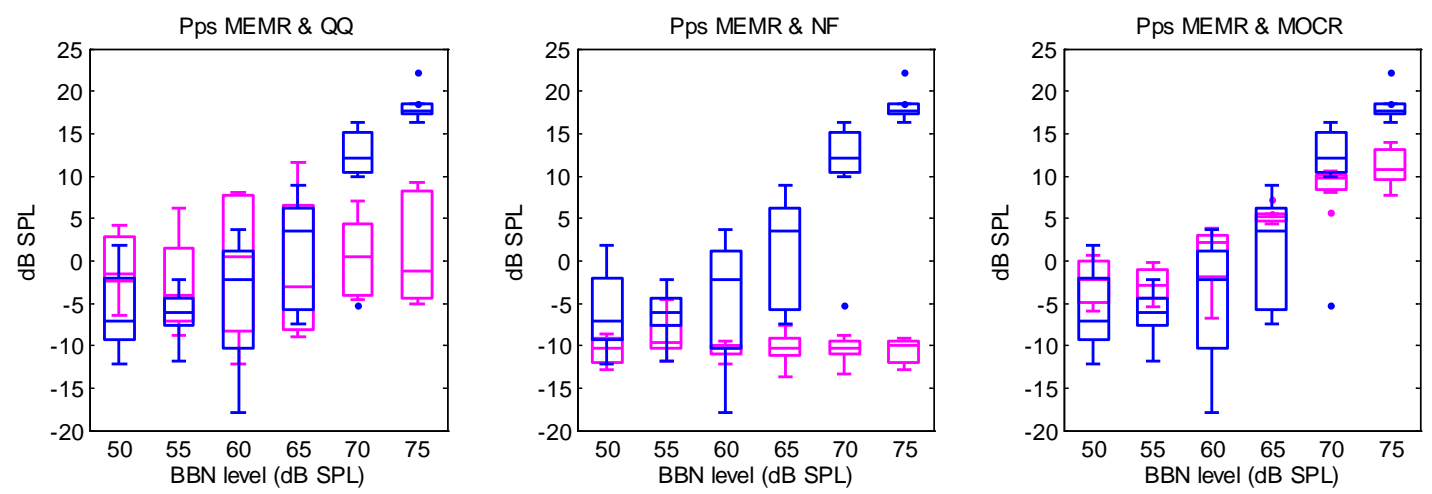

For Subject 287L, we can clearly see a detectable reflex at 70 and $75 \mathrm{~dB}$ SPL, but variability is high across all levels so we cannot safely say there is no reflex at $65 \mathrm{~dB}$ SPL or lower. Of concern is that the MOCR magnitude is equal to the MEMR magnitude and its increasing I/O function may be entirely due to MEMR. This subject did not continue in the experiment.

\section{Subject ID 265, L Ear}
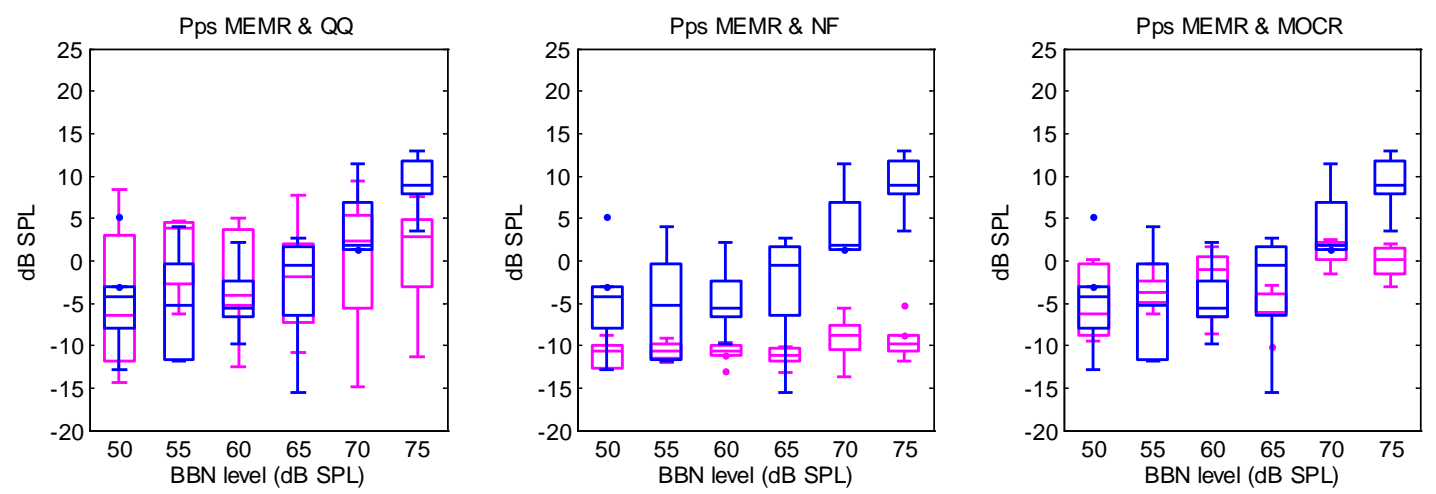

For Subject 265L, there is a detectable reflex at 75, but again variability is high across all levels so we cannot safely say there is no reflex at lower levels. Because the variability is masking the MEMR, we need to consider if a $5 \mathrm{~dB}$ SPL MEMR magnitude could affect the MOCR. The raw MOCR in this case is small at only around $0 \mathrm{~dB}$ SPL, so it could be possible that it is affected by the larger MEMR (if it exists). The subject was not included in the MOCR experiment. 


\section{Subject ID 217, R Ear}
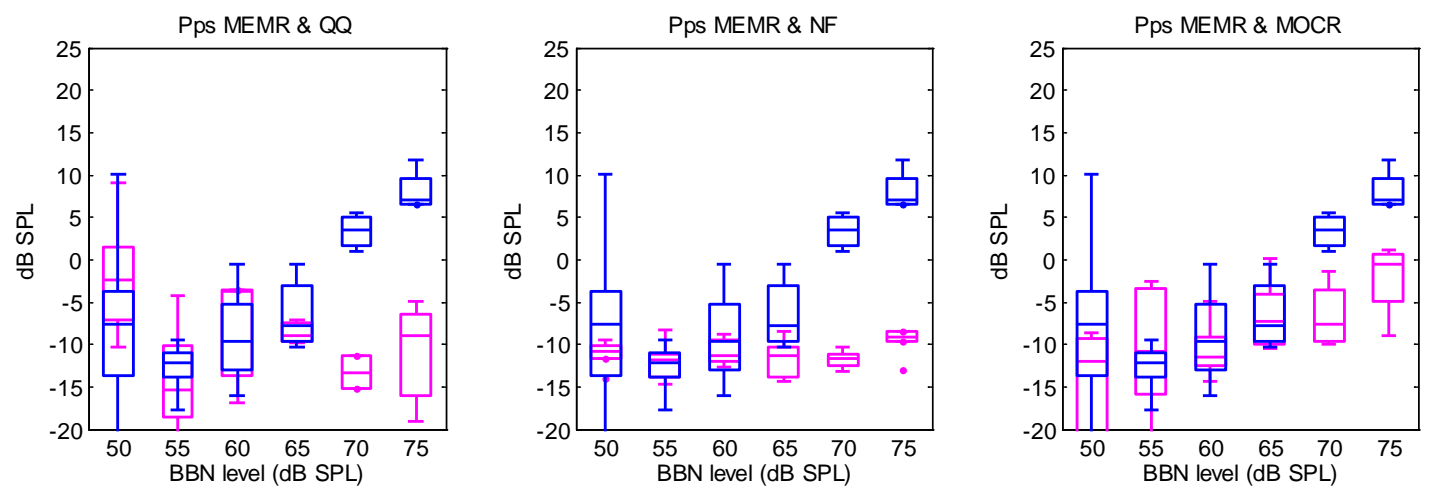

For Subject 217R, despite variable results at $50 \mathrm{~dB}$ SPL, we can ascertain that 55-65 dB SPL is safe for testing MOCR because variability is low at these levels. Variable results at just one or two levels are commonly seen, which can make MEMR threshold determination difficult. The MOCR is low and its increase at $75 \mathrm{~dB}$ SPL is possibly due to the MEMR which is >> MOCR.

\section{Subject ID 278, R Ear}
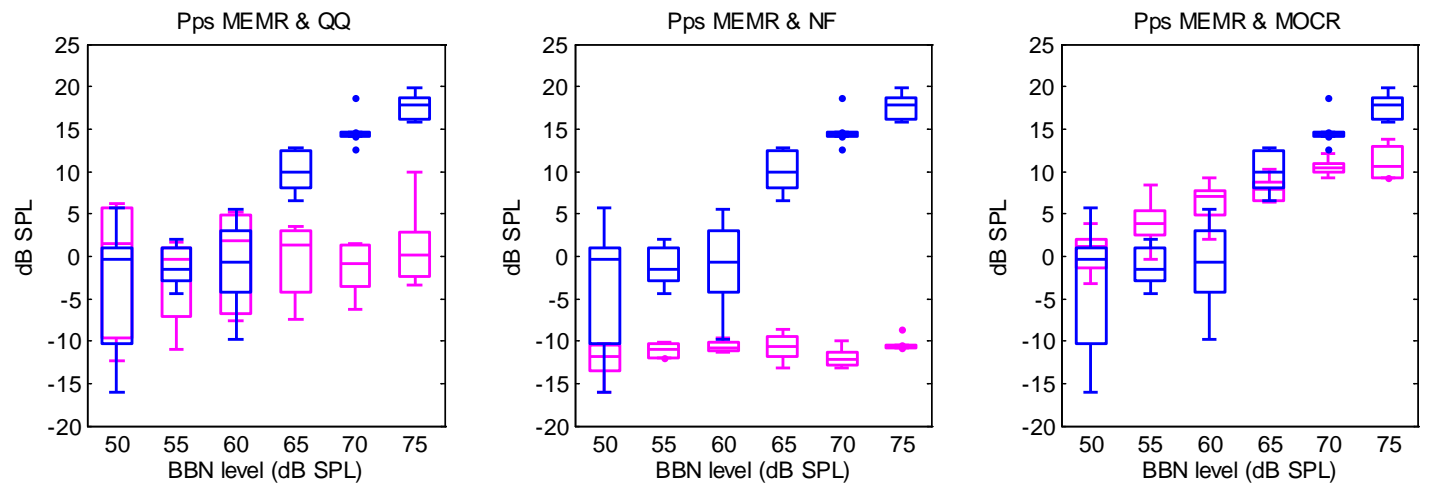

Partway through the experiment, we started allowing results like this where there was clearly a reflex at 65 and higher and the MEMR was showing a sigmoidal increase with BBN level, but lower levels had high variability ( $>0 \mathrm{~dB}$ SPL) - we allowed up to $5 \mathrm{~dB}$ SPL. In hindsight this might not be the greatest idea because the MEMR variability could be masking a small reflex at $60 \mathrm{~dB}$ SPL. The MOCR increases throughout the I/O function - typically we see this through 50-60 dB SPL even when there is no MEMR and here the results are potentially confounded. In this case the subject didn't continue, but for reasons other than poor MEMR results. 


\subsection{Ambiguous results - Variability High even when Noise is low Subject ID 209, L Ear}
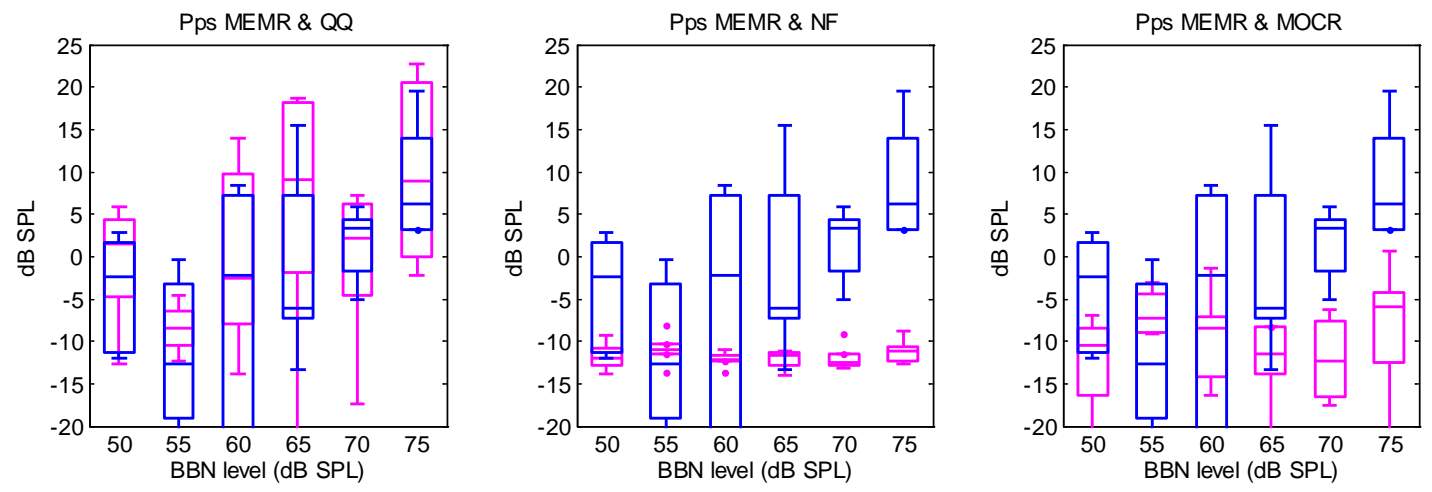

Subject 209L is all too typical. Only one or two levels give clear results, and they may or may not allow us to deduce that $60 \mathrm{~dB}$ SPL is safe for MOCR testing. Here we can only say that $55 \mathrm{~dB}$ SPL is sufficient; although with the lowish results at 70, it is likely that $60 \mathrm{~dB}$ SPL is safe. There is no MOCR measurable at $1 \mathrm{kHz}$ to gauge MEMR effects. This subject did not continue due to these variable MEMR and MOCR results despite being a quiet subject to test.

\section{Subject ID 250, R Ear}
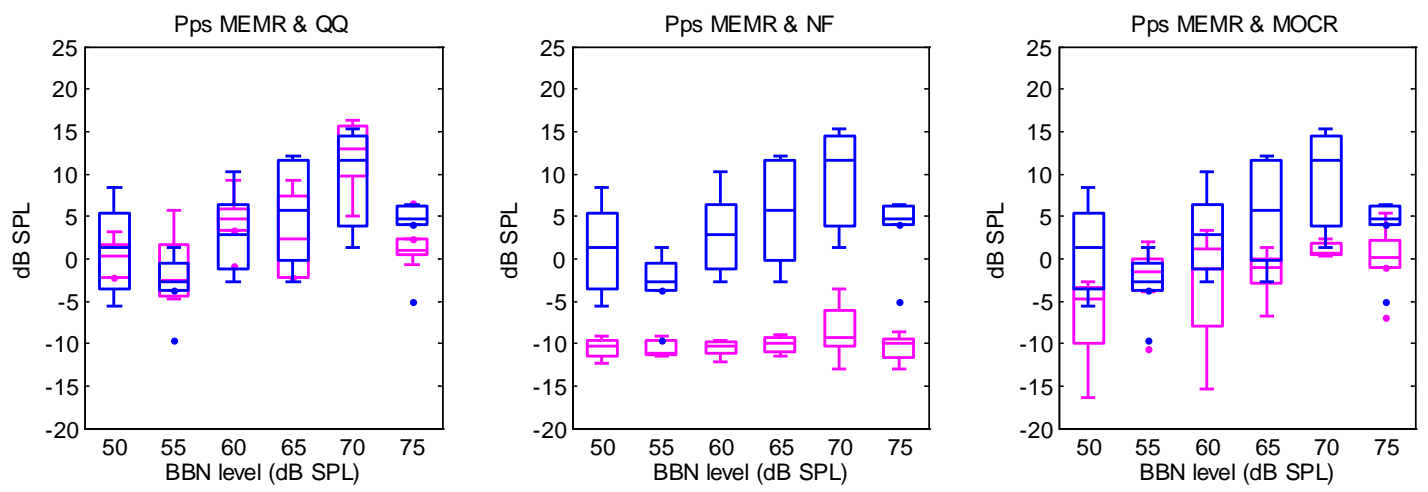

Noise levels for Subject 250R are low, but variability is too high, precluding establishing MEMR threshold except perhaps at $75 \mathrm{~dB}$ SPL. The MOCR plateaus at $60 \mathrm{~dB}$ SPL like it does for many subjects. Note that although the MEMR itself might interact with the MOCR, the variability inherent to the MEMR doesn't directly affect the MOCR magnitude. Due to the nature of the underlying calculations, the pressure fluctuations at $f_{p}$ are too slow to contribute to variability in the derivation of the SFOAE, which underlies the MOCR. 


\section{Subject ID 231, R Ear}
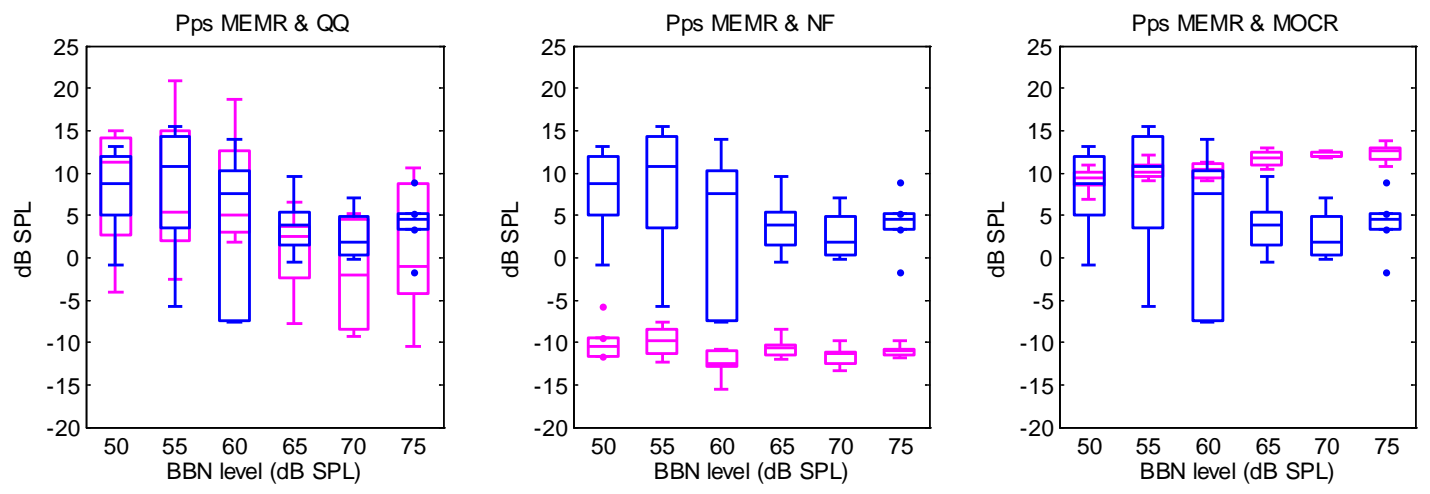

Subject 231R shows high variability, especially at lower levels. If there is a MEMR, it isn't large. The MOCR, however, is large but plateaus around $65 \mathrm{~dB}$ SPL. MOCR $>>$ MEMR so it is unlikely the MOCR was confounded with MEMR effects. The subject did not continue because of these unclear results, but in hindsight it possibly would have been safe to include her.

\section{Subject ID 240, L Ear}
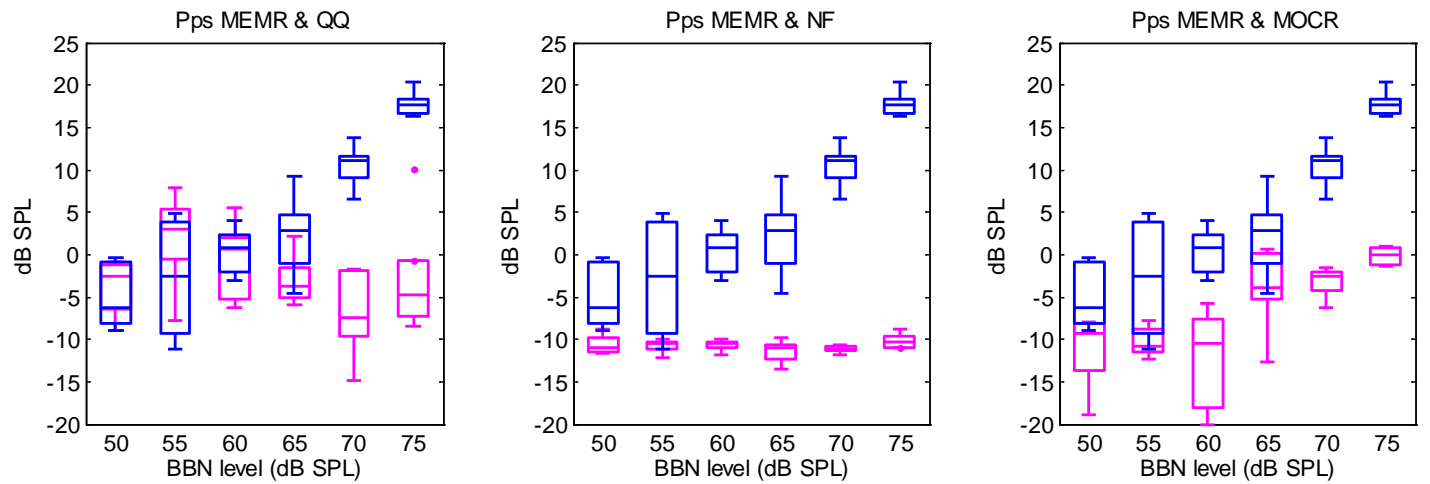

\section{Subject ID 240, R Ear}
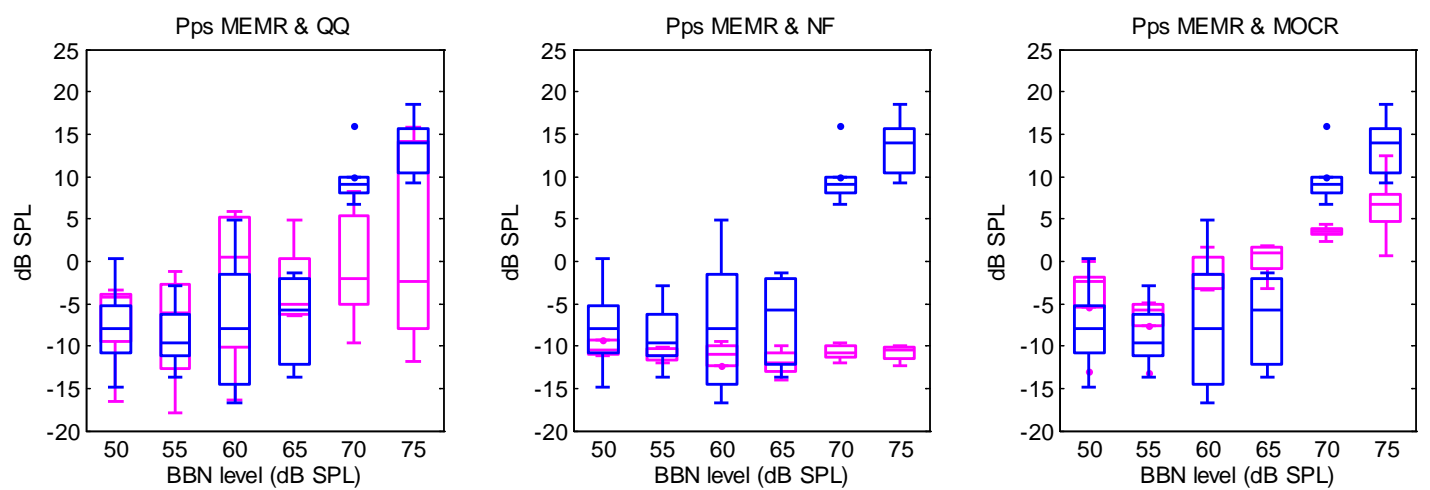

For both ears of Subject 240, the MEMR is clearly distinguishable from the noise floor, but variability precludes an assessment of whether $60 \mathrm{~dB}$ SPL is safe for MOCR testing. Very low MOCR 
(especially for the left ear) rises with MEMR, suggesting confounding with MOCR $<<$ MEMR. The subject did not continue in the experiment.

\section{Subject ID 232, L Ear}
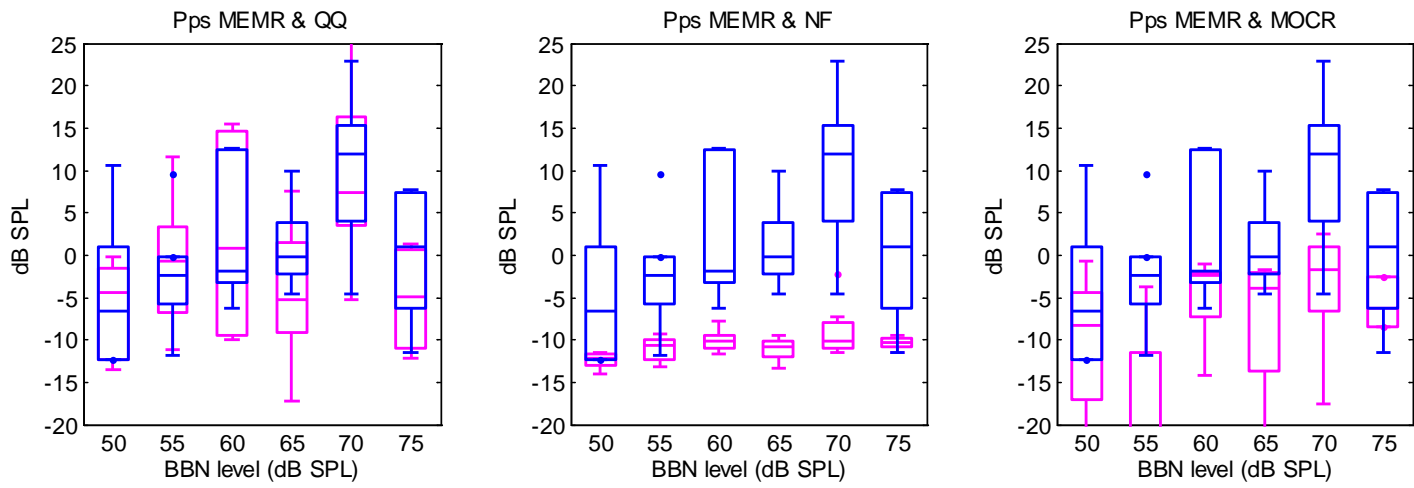

For Subject 232L, the MEMR cannot be reliably detected at any level.

\section{Subject ID 237, R Ear}
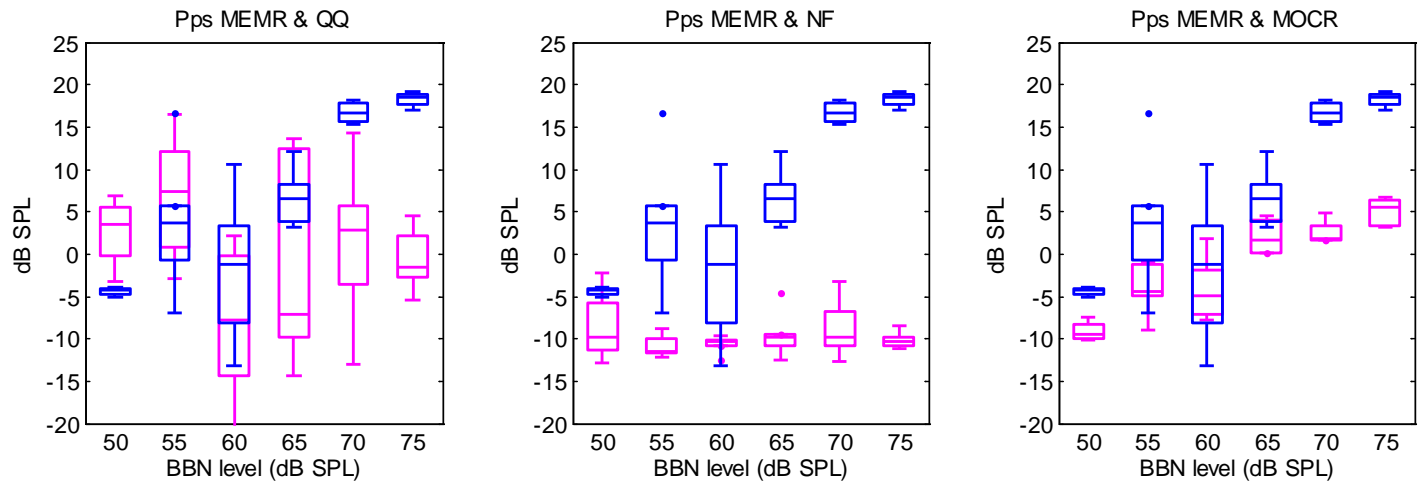

For Subject 237R, there is a reflex in among the variability, but it can only be reliably detected at 70 and $75 \mathrm{~dB}$ SPL. It is unclear if the MOCR is affected. 


\subsection{Worst case scenario}

\section{Subject ID 288, L Ear}
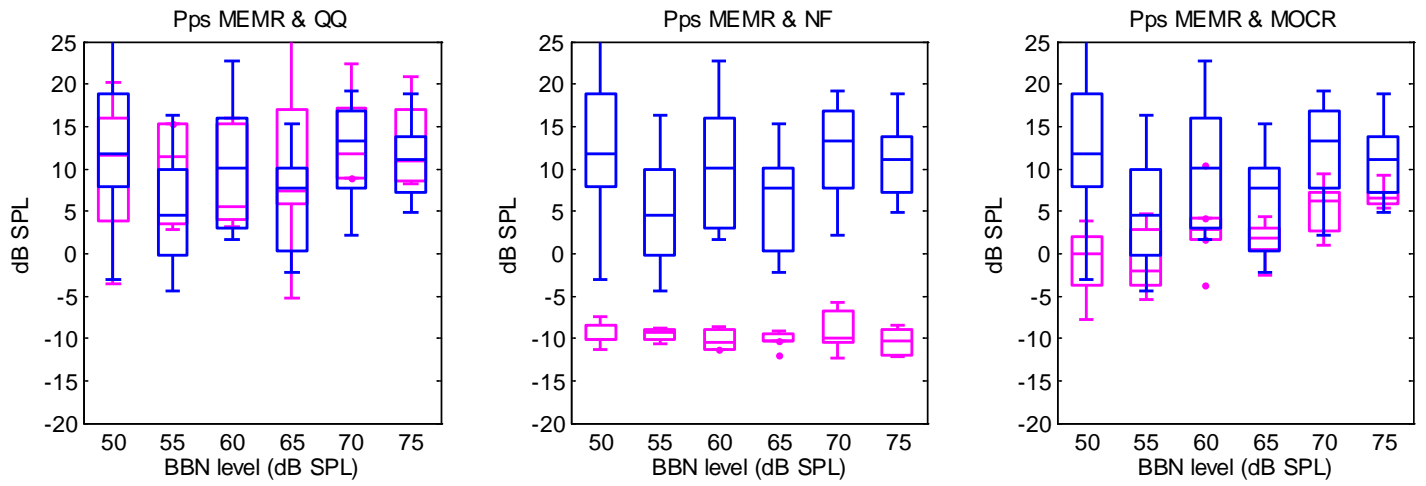

Subject 288 is a worse-case scenario. No levels are clear, and we cannot deduce much. The MOCR plateaus at 60-65 dB SPL but then increases at 70-75 dB SPL, suggesting that maybe there is a confounding MEMR at the higher levels. Results are not usually this bad. This subject was very fidgety, and we didn't continue with her. 


\section{Appendix D: Method 3 MOCR and MEMR}

This appendix shows four examples (from over 300) for the MEMR derived from MOCR measurements using SFOAEs for individual measurement series (see Section 7.5). These plots show that MEMR activity can vary across a test session, yet not necessarily affect the MOCR. It is hard to come to an overall conclusion about how MEMR interacts with MOCR from these plots, but it provides some examples of how it can fluctuate throughout a test.

- Top left: SFOAE, raw MOCR, \& MEMR magnitudes by trial-pair. Raw MOCR is an estimate of MOCR strength prior to normalization and has the same units (dB SPL) to the SFOAE and MEMR, allowing direct comparisons.

o SFOAE_Quiet, sky blue circles that are solid if quality criteria are met.

o SFOAE_Noise, brick red circles that are solid if quality criteria are met.

o MOCR, lavender triangles that are solid if quality criteria are met.

o MEMR, forest green triangles that are solid if quality criteria are met.

o Corresponding noise floors are dashed lines using same colors.

- Top right: SFOAE phase by trial-pair

o SFOAE_Quiet, sky blue circles that are solid if quality criteria are met.

o SFOAE_Noise, brick red circles that are solid if quality criteria are met.

- Middle left: raw MOCR \& MEMR by trial-pair (dB SPL), showing the same data as above, along with associated inherent test variability, uncluttered by the SFOAEs and the noise floors.

o MOCR, lavender triangles that are solid if quality criteria are met.

o MOCR variability, lavender-gray triangles with dashed lines, calculated from paired Q and paired $\mathrm{N}$ trials. Plotted with an offset when there is 1 less trial.

o MEMR, forest green triangles that are solid if quality criteria are met.

o MEMR variability, green-gray triangles with dashed lines, calculated from paired Q trials only. Plotted with an offset when there is 1 less trial.

- Middle right: box and whisker plots collapsed over trial-pair, corresponding to the data displayed in the middle-left plot.

o MOCR, lavender box; only includes data meeting quality criteria.

o MOCR variability, lavender-gray box; only includes trials meeting quality control. No box plotted if there are fewer than 5 good trials.

o MEMR, forest green box that only includes data meeting quality criteria.

o MEMR variability, green-gray box; only includes trials meeting quality control. No box plotted if there are fewer than 5 good trials.

- Bottom left: MOCR strength as a percentage by trial-pair. MOCR\% is the raw MOCR normalized by the OAE magnitude.

o MOCR, lavender triangles that are solid if quality criteria are met. Error bars are 95\% CI based on the standard error of the mean.

o MOCR variability, lavender-gray triangles with dashed lines (as above).

o Mean MOCR\%, horizontal line in lavender.

- Bottom right: box and whisker plots collapsed over trial-pair, corresponding to the data displayed in the bottom-left plot.

o MOCR\%, lavender box; only includes data meeting quality criteria. 
o MOCR\% variability, lavender-gray box; only includes trials meeting quality control. No box plotted if there are fewer than 5 good trials. 

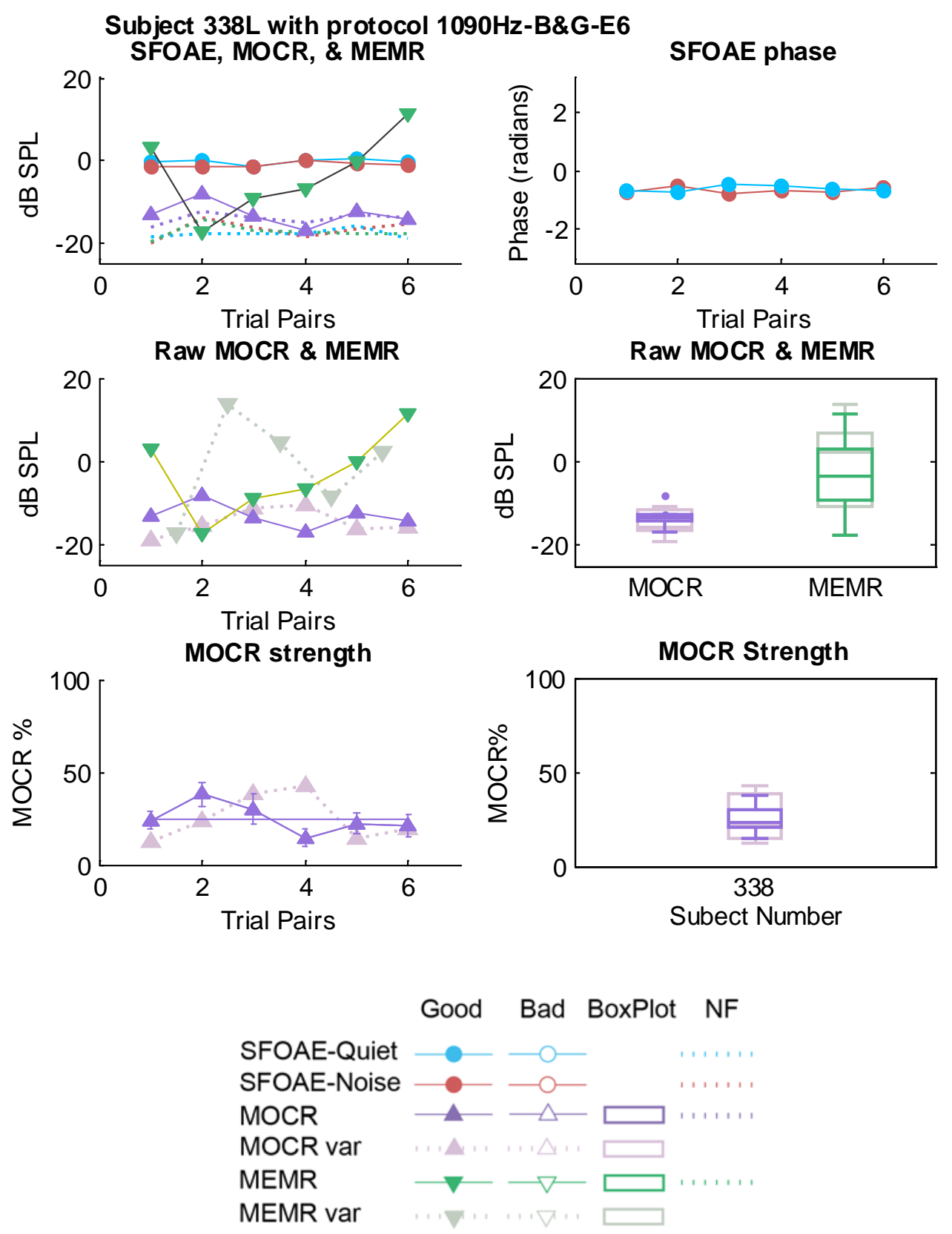

In this example, the MEMR is potentially masked by variability, and could be much larger than the raw MOCR (but we can't tell). It is possible that the trials where the MEMR is larger are associated with trials where the MOCR is lower, and that the MEMR is partially responsible for the MOCR variability as it might not be engaging on every trial. 

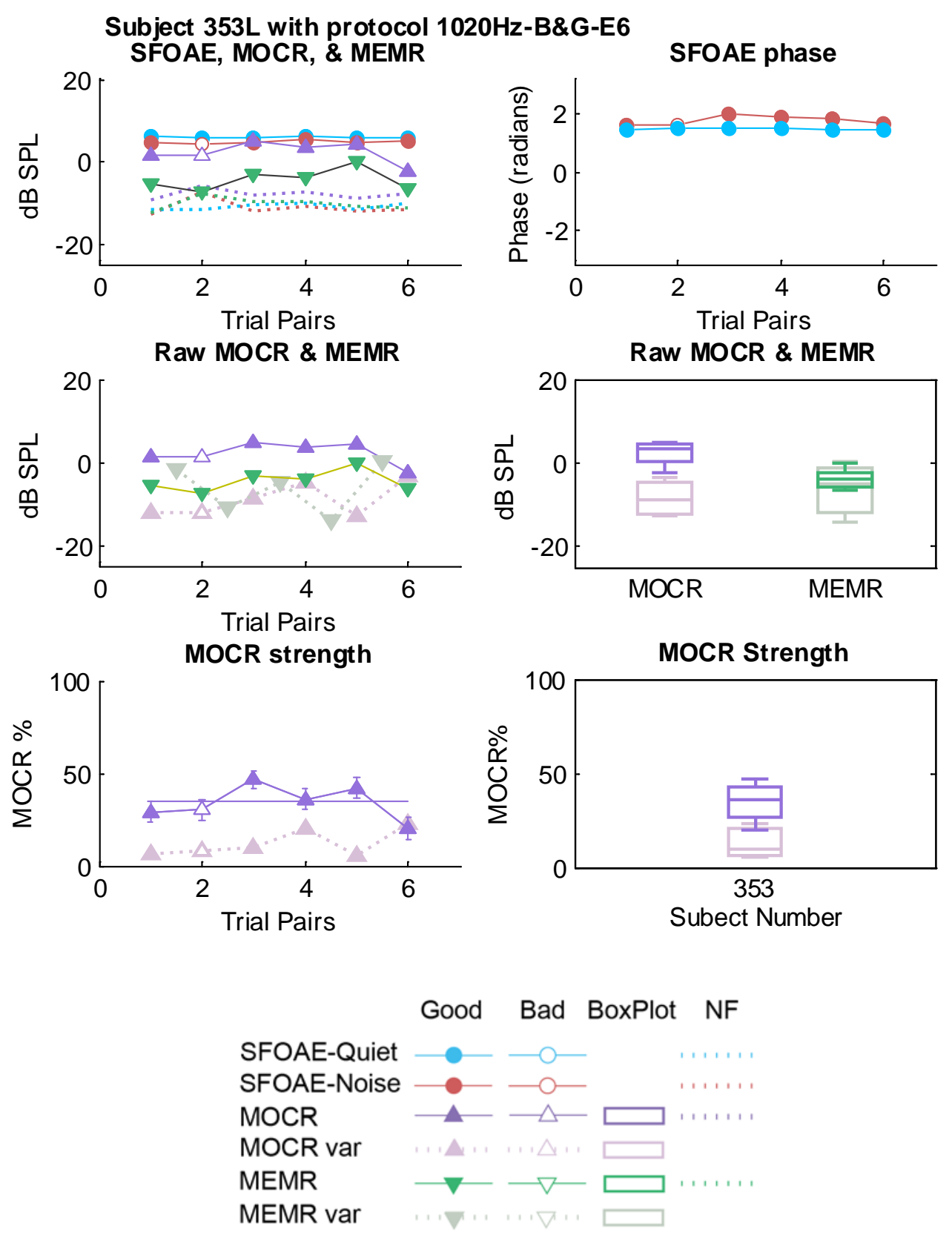

In this example an MOCR is detectable from its inherent variability. The MEMR is not detectable, but the MEMR and MEMR variability is lower than the raw MOCR, so even if the MEMR is masked, it is unlikely to affect the MOCR. 

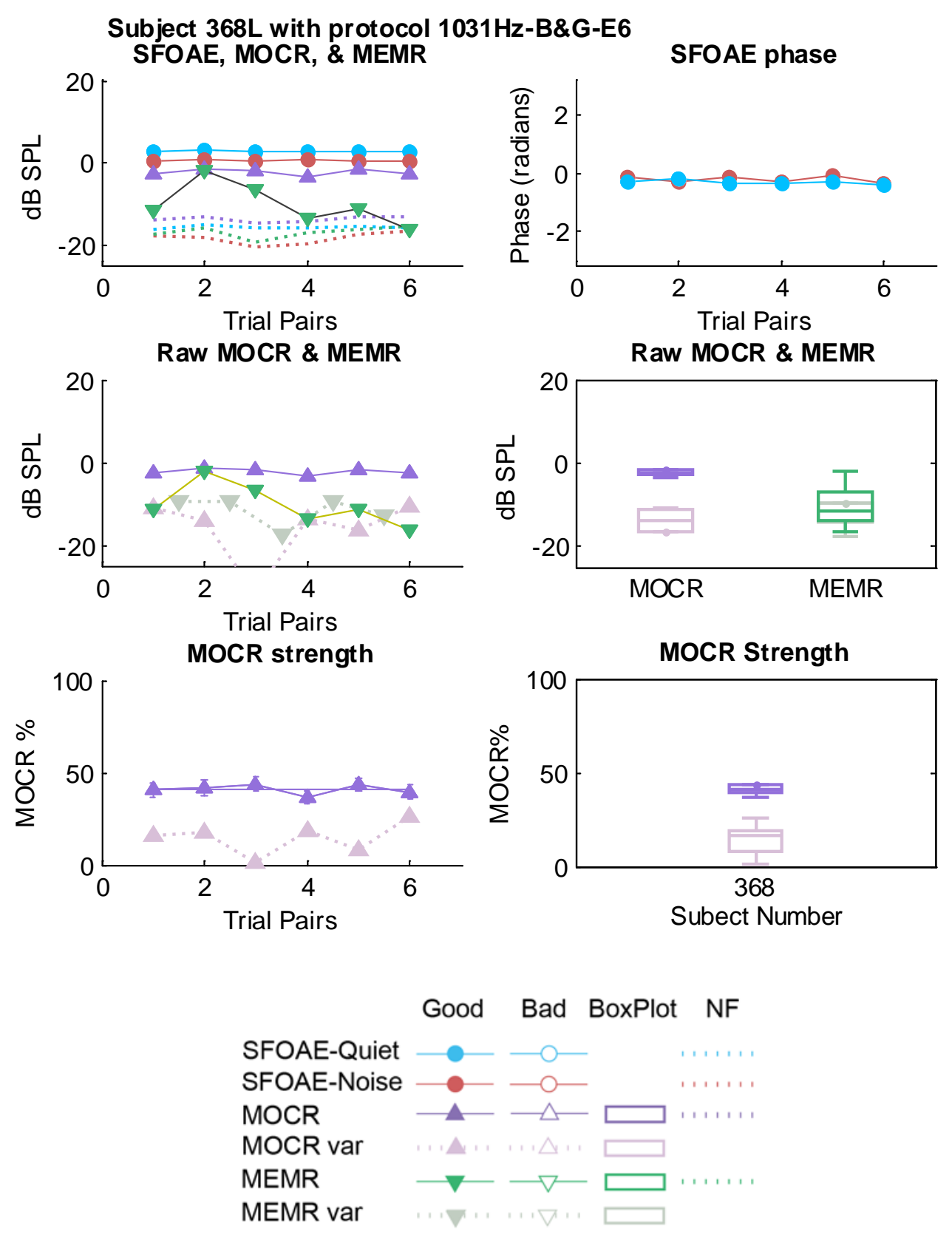

In this example, the MOCR is strong and stable, and clearly detectable from its inherent variability. The MEMR is mostly much lower than the raw MOCR except for one outlier in trial 2, which is of the same magnitude as the raw MOCR. In this case it does not appear to have affected the MOCR measurement. 

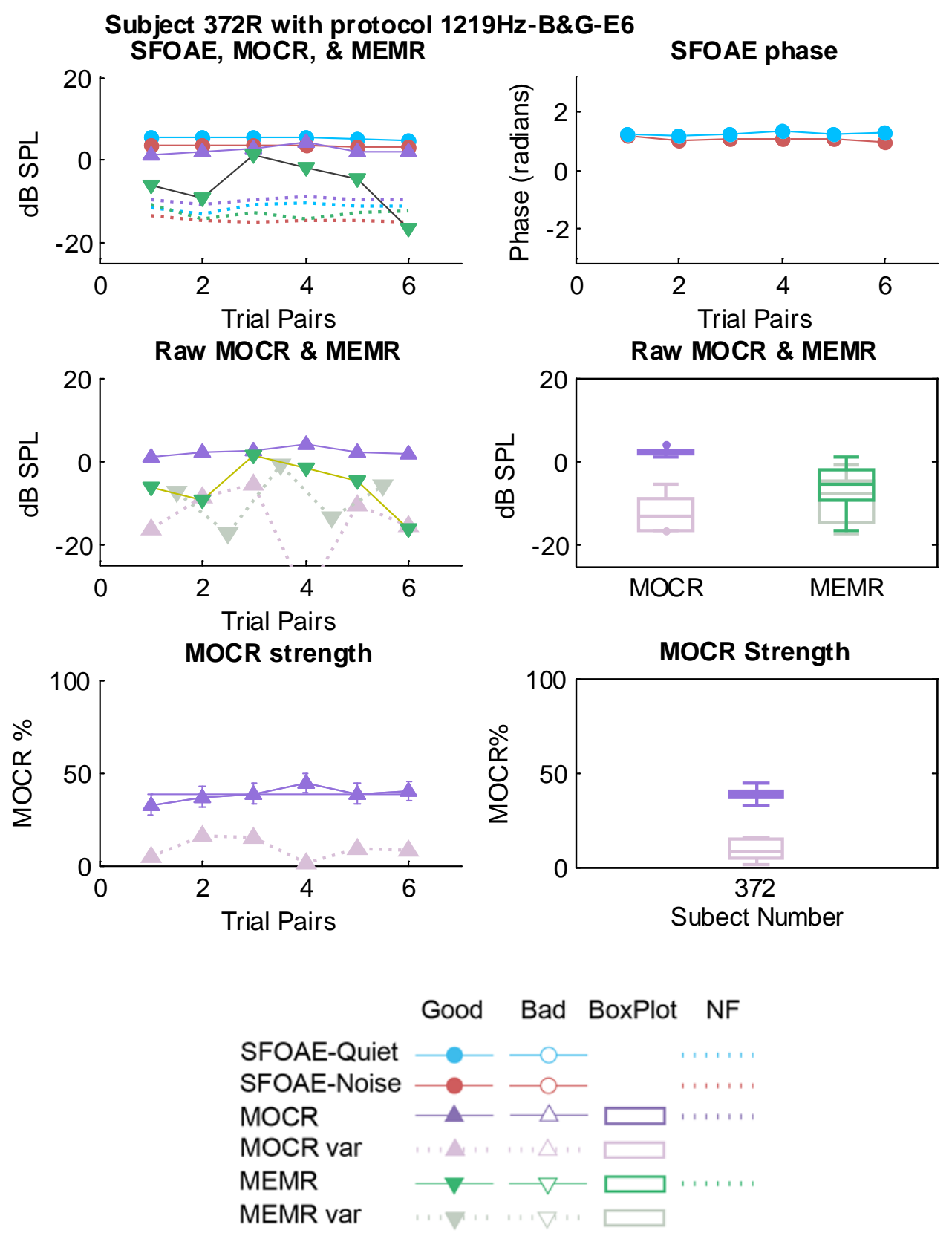

In this example, the MOCR is strong and stable, and clearly detectable from its inherent variability. The MEMR is mostly much lower than the raw MOCR except for one outlier in trial 3, which is of the same magnitude as the raw MOCR. In this case it does not appear to have affected the MOCR measurement. 


\section{Appendix E: Method 3 MOCR and MEMR box plots}

As a second pass at evaluating MEMR derived from the MOCR measurements, MEMR and MOCR box plots were taken from the individual summary graphs shown in Appendix D (middle right and middle bottom plots) as a function of frequency, with a comparison to the inherent test variability (by comparing adjacent Q trials where there was no contralateral MEMR elicitor; see Section 7.5). The box plots are drawn so that frequency is spaced accurately (frequency range is plotted from min to max for each individual ear), with the downside that labels can overlap. These graphs are summarized in Table 1 on page 17. A MEMR was considered not measurable if it was inseparable from the inherent variability. A MEMR was considered not present if it was below -5dB SPL and was inseparable from the inherent variability. In no case was MEMR activity sufficiently separable from the inherent variability to clearly show that the reflex had engaged.

MEMR: Top plot

- MEMR, forest green box that only includes data meeting quality criteria.

- MEMR variability, green-gray box, calculated from paired Q trials only.

Raw MOCR: middle plot

- MOCR, lavender box that only includes data meeting quality criteria.

- MOCR variability, lavender-gray box, calculated from paired Q and paired N trials.

MOCR\%: bottom plot

- MOCR, lavender box that only includes data meeting quality criteria.

- MOCR variability, lavender-gray box, calculated from paired Q and paired N trials.

Box plots

- These "compact" style box plots show the interquartile (IQR) range as a thick bar, the median as a circle with a black dot, whiskers as thin bars, and outliers as small circles. If there is more than one outlier, they are slightly offset from one another. The whiskers extend to the furtherest data point within 1.5xIQR, or to 1.5xIQR if there are also outliers.

Please note that the following data are NOT designed to show how the MOCR may vary with frequency because the frequencies were not optimally chosen for this purpose. They can, however, be used to see how a MEMR may affect the MOCR. Although no MEMR was large and detectable (separated from its inherent variability), there are many cases where the variability was so great that a MEMR with moderate magnitude have been masked. 
Subject 338L
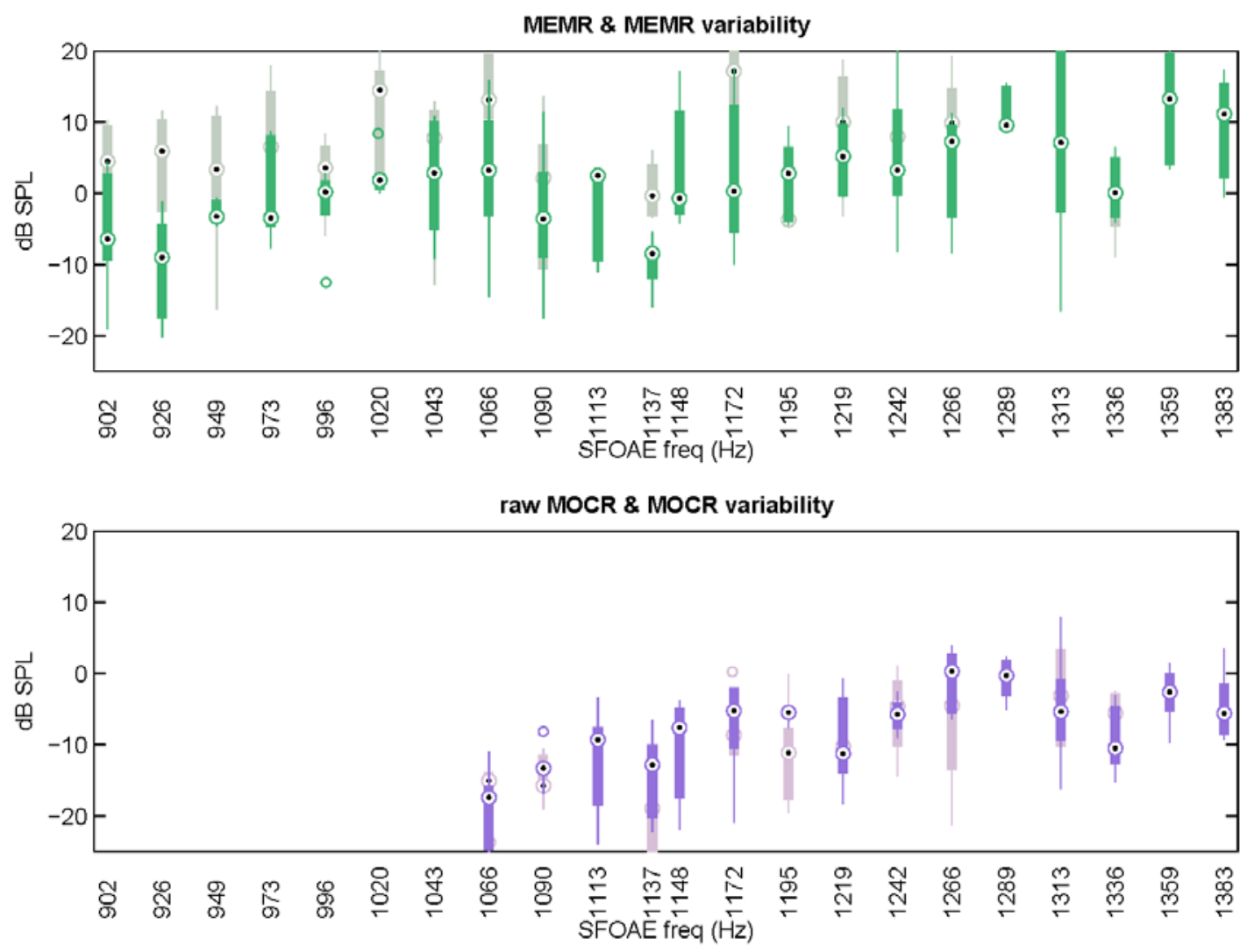

MOCR strength \& variability

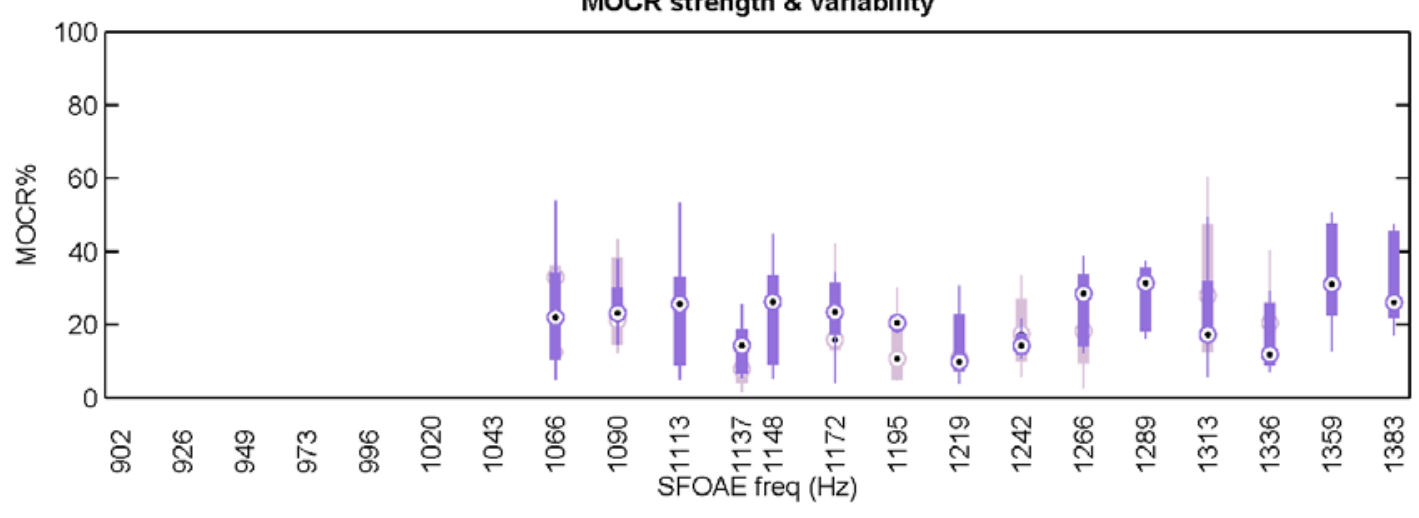

For S338L, frequencies below $1 \mathrm{kHz}$ did not produce measurable MOCR due to low-level OAEs, and at other frequencies the MOCR was low and undetectable from variability. MEMR variability was high across all frequencies, and potentially could be masking MEMR activity. The order of magnitude of the MEMR variability was higher than the raw MOCR, meaning any interaction with the MOCR could potentially affect the results. 


\section{Subject 353L}
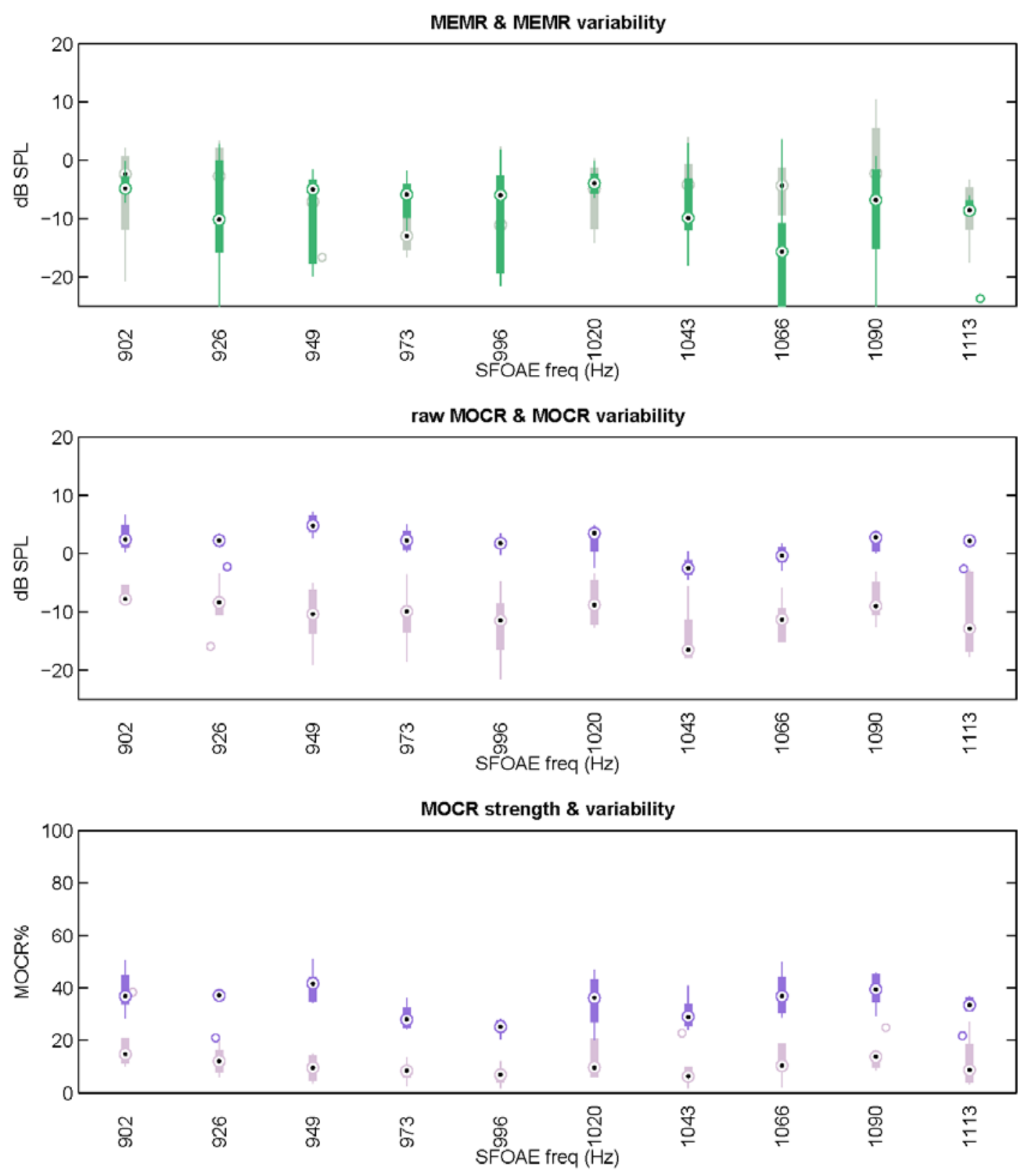

For S353L, only the " $1 \mathrm{kHz}$ ” frequencies were tested. The MOCR was relatively constant in this narrow frequency range, and detectable. MEMR variability was moderate, but mostly at a lower level than the raw MOCR so is unlikely to have affected the results. 


\section{Subject 368L}
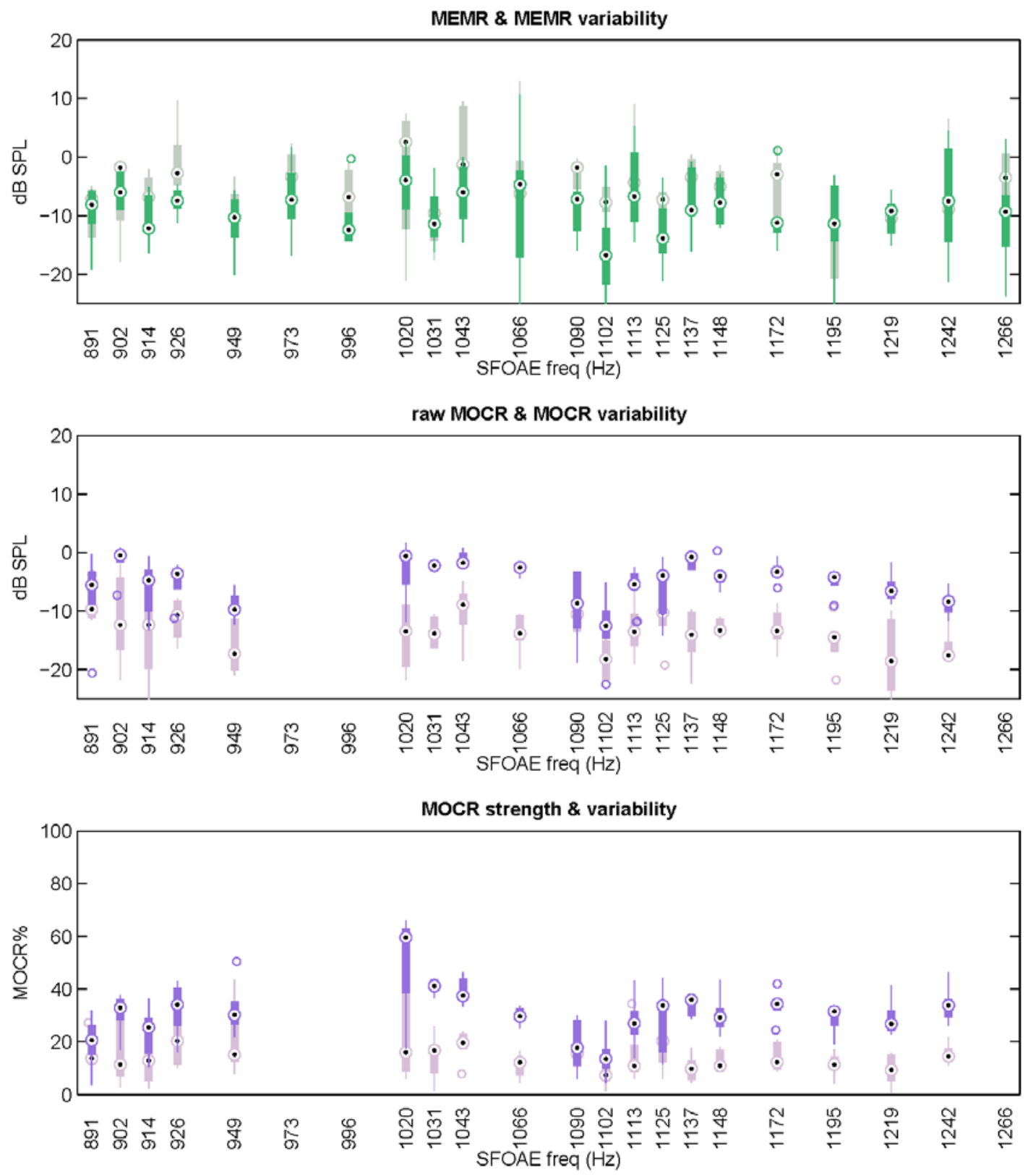

For S368L, the "Best” MOCR frequencies were interspersed and slightly above the $1 \mathrm{kHz}$ band of frequencies, due to SOAEs at higher frequencies. The MOCR is not readily detectable below $1 \mathrm{kHz}$, and there are some missing data. Above $1 \mathrm{kHz}$ the more variable MOCR frequencies are associated with dips in the OAE spectrum. MEMR activity and associated variability was low at some frequencies. At other frequencies the variability was moderate to high, but there wasn't a pattern across frequency, nor did the resulting MOCRs necessarily differ from their less-variable neighbors. 


\section{Subject $370 R$}
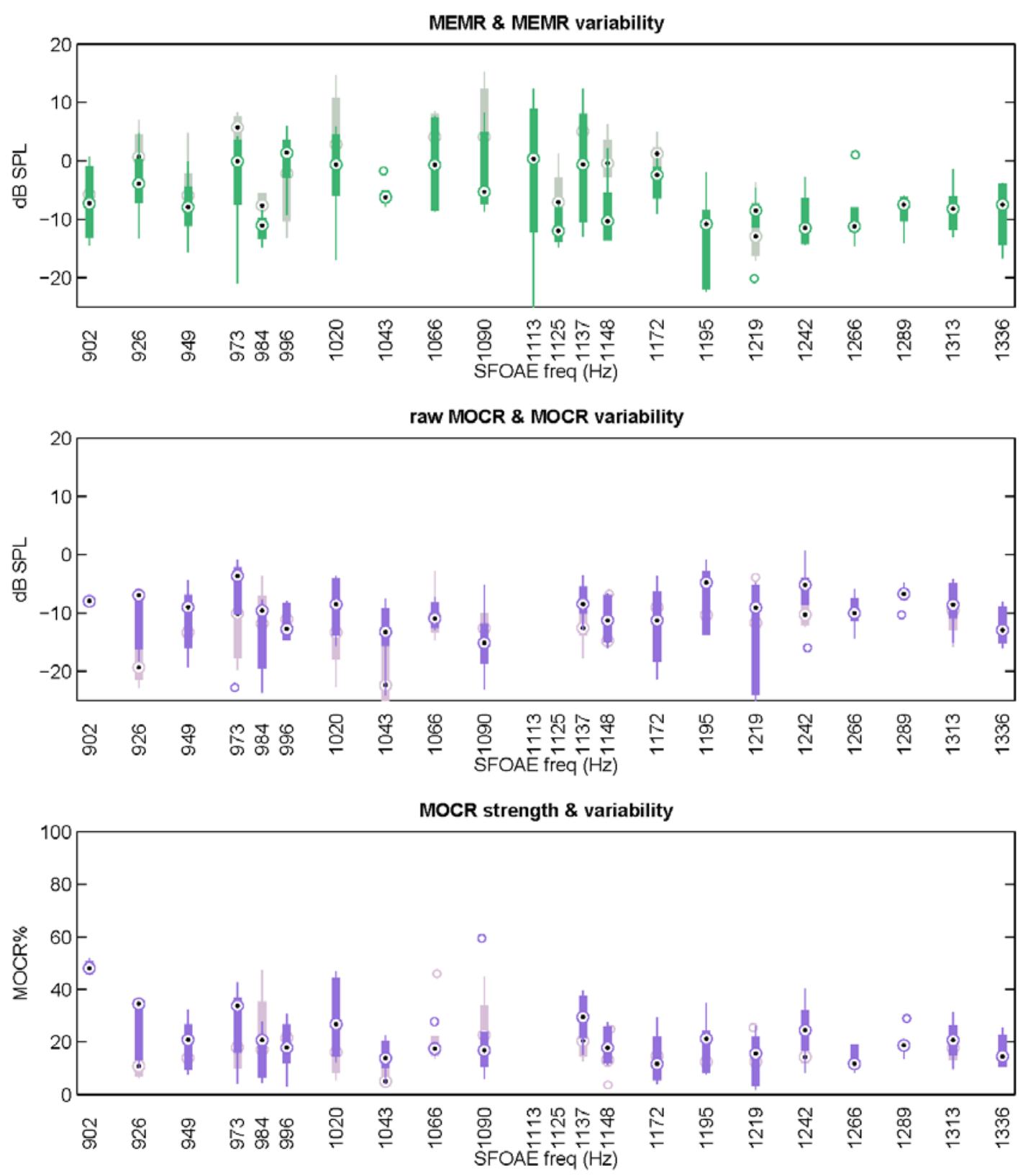

S370R has low MOCR strength that is not separable from inherent test variability, and there are some missing data. Could the low MOCR be due to MEMR activity? Below $1.2 \mathrm{kHz}$, MEMR variability is very high (much higher than the raw MOCR activity) so it is not possible to tell if a small MEMR reflex is interfering. There may also be interference from a small SOAE that had not met criteria for exclusion in screening. At higher frequencies (and a couple of lower ones), the MEMR variability is low, but the MOCR is similar. These frequencies were tested in a different session. This indicates that the degree of MEMR variability is not necessarily consistent over time, and this reinforces the desirability for testing MEMR at the same time as the MOCR measurement. In this case, it appears there is no latent MEMR because MOCR is similar for frequencies with high and low MEMR variability. 


\section{Subject 371L}
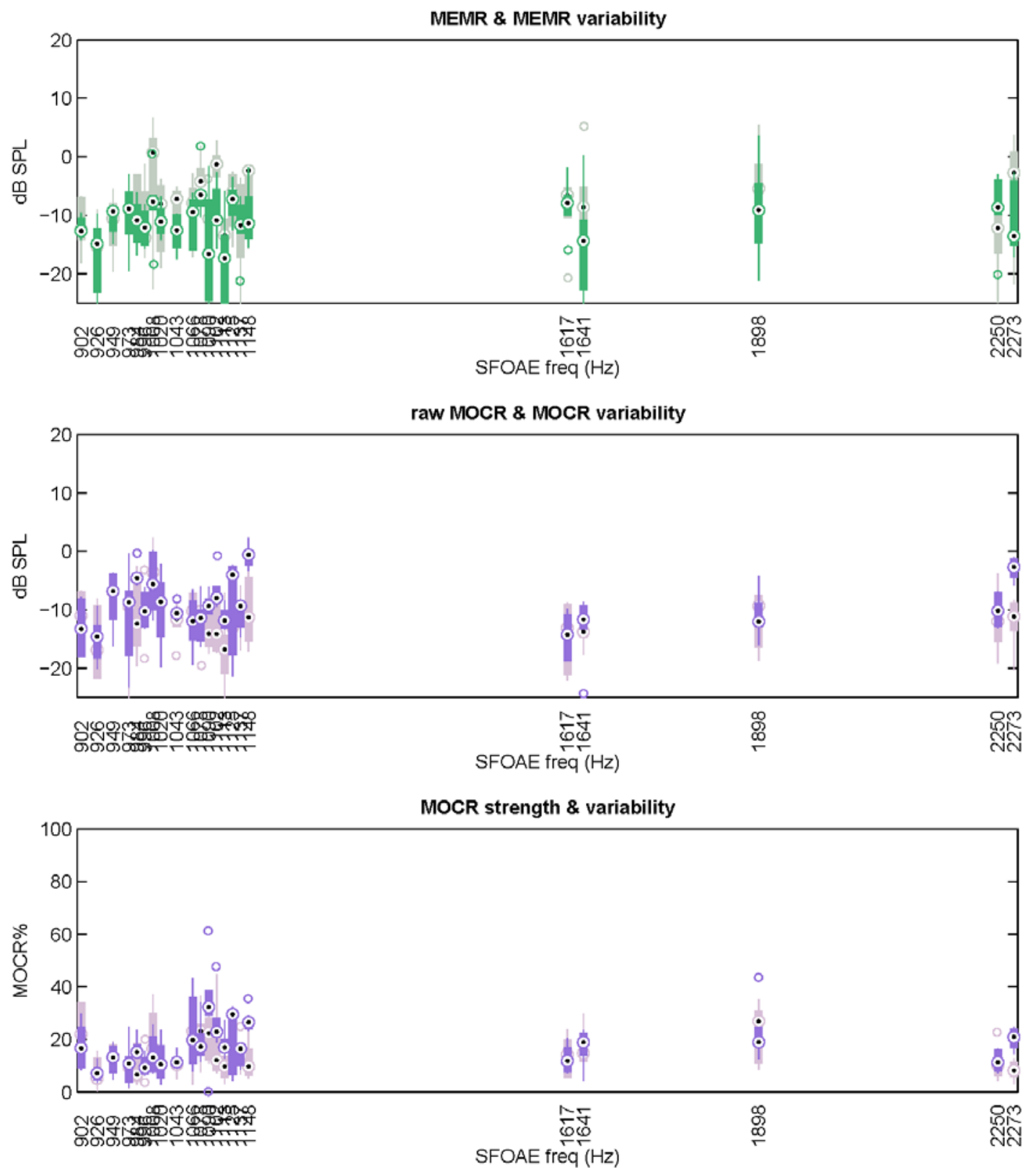

S371L has a detectable MOCR only at a couple of frequencies. Unusually, this ear had both SOAEs and areas with low-level OAEs, making it difficult to find good frequencies for testing. MEMR activity and variability is low at many frequencies, so it is unlikely the undetectable MOCR are due to the MEMR. Compare these results with those in the right ear on the next page. 


\section{Subject 371R}

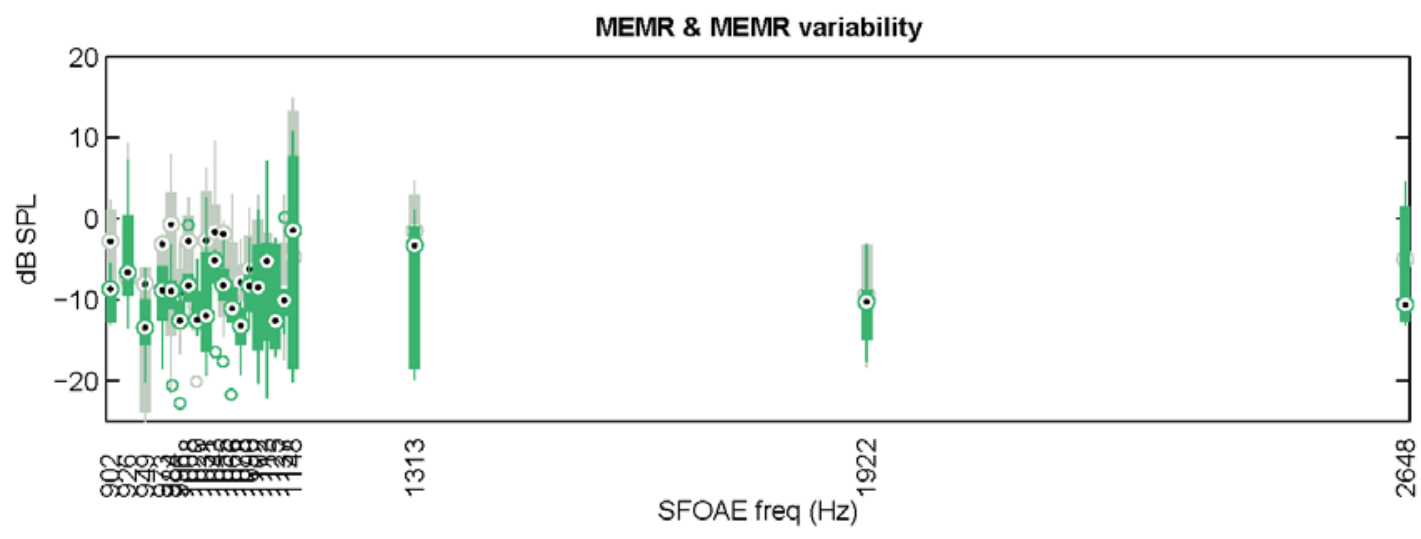

raw MOCR \& MOCR variability

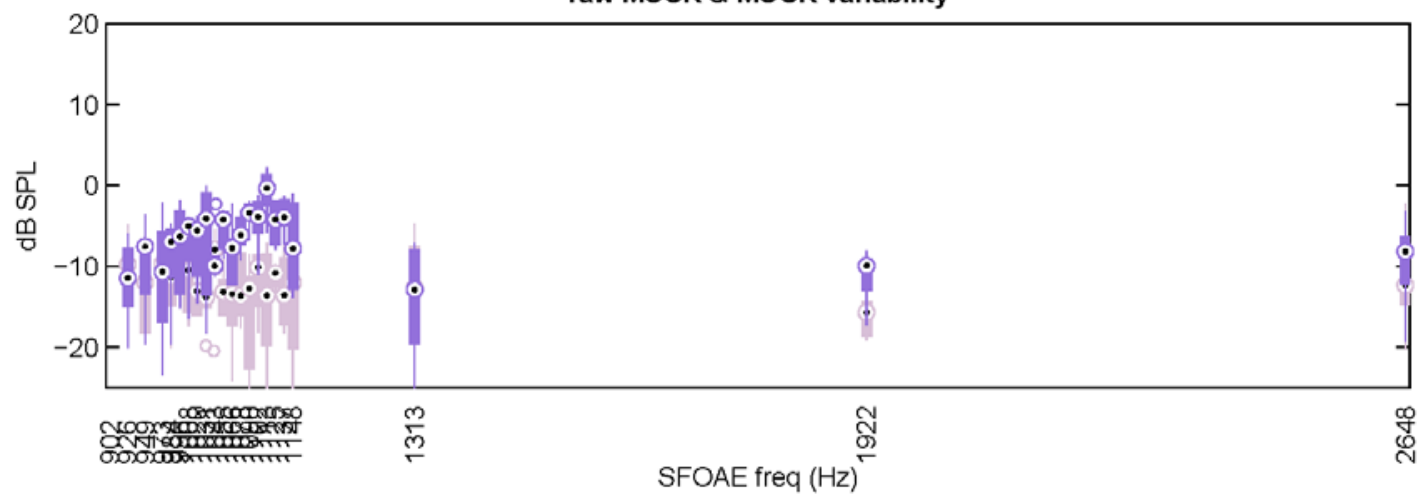

MOCR strength \& variability

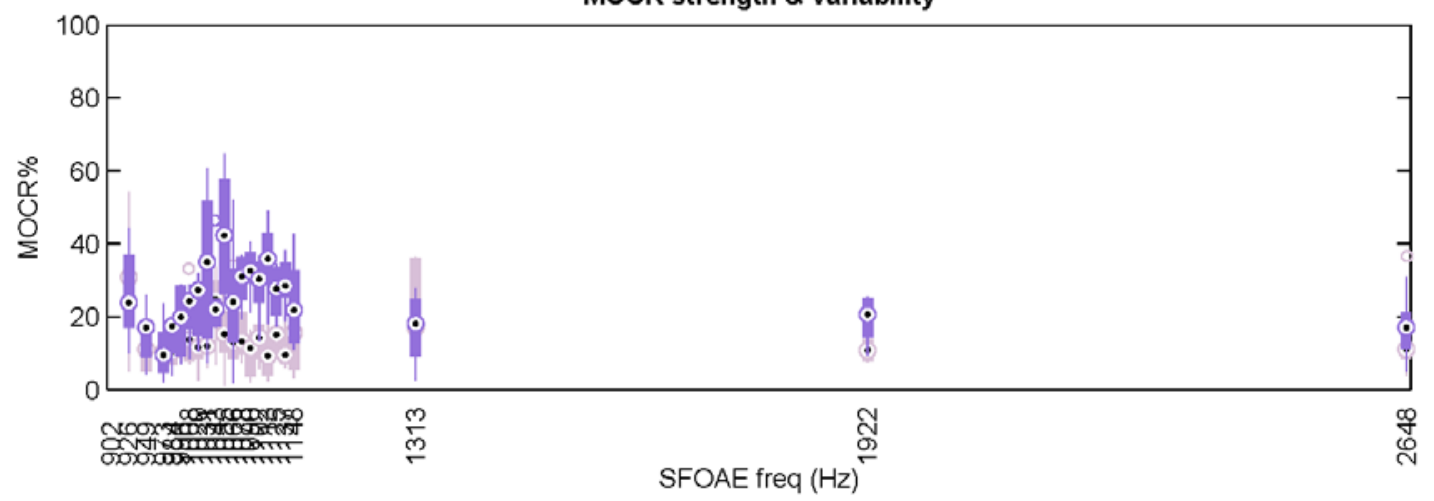

S371R's "Best” frequency range overlapped with the $1 \mathrm{kHz}$ range above $1 \mathrm{kHz}$. It is apparent that the ear has a moderately strong, clearly detectable MOCR around 1-1.1 kHz. Below $1 \mathrm{kHz}$, and at the higher frequencies, the MOCR is not detectable, due to low-level OAEs that only just met screening criteria. Like the left ear, this ear also has the unusual combination of low-level OAEs and SOAEs. This ear also shows moderately high MEMR variability, though through the region where MOCR is strongest, it is not as high, indicating the results at those frequencies are probably valid. 


\section{Subject 372R}
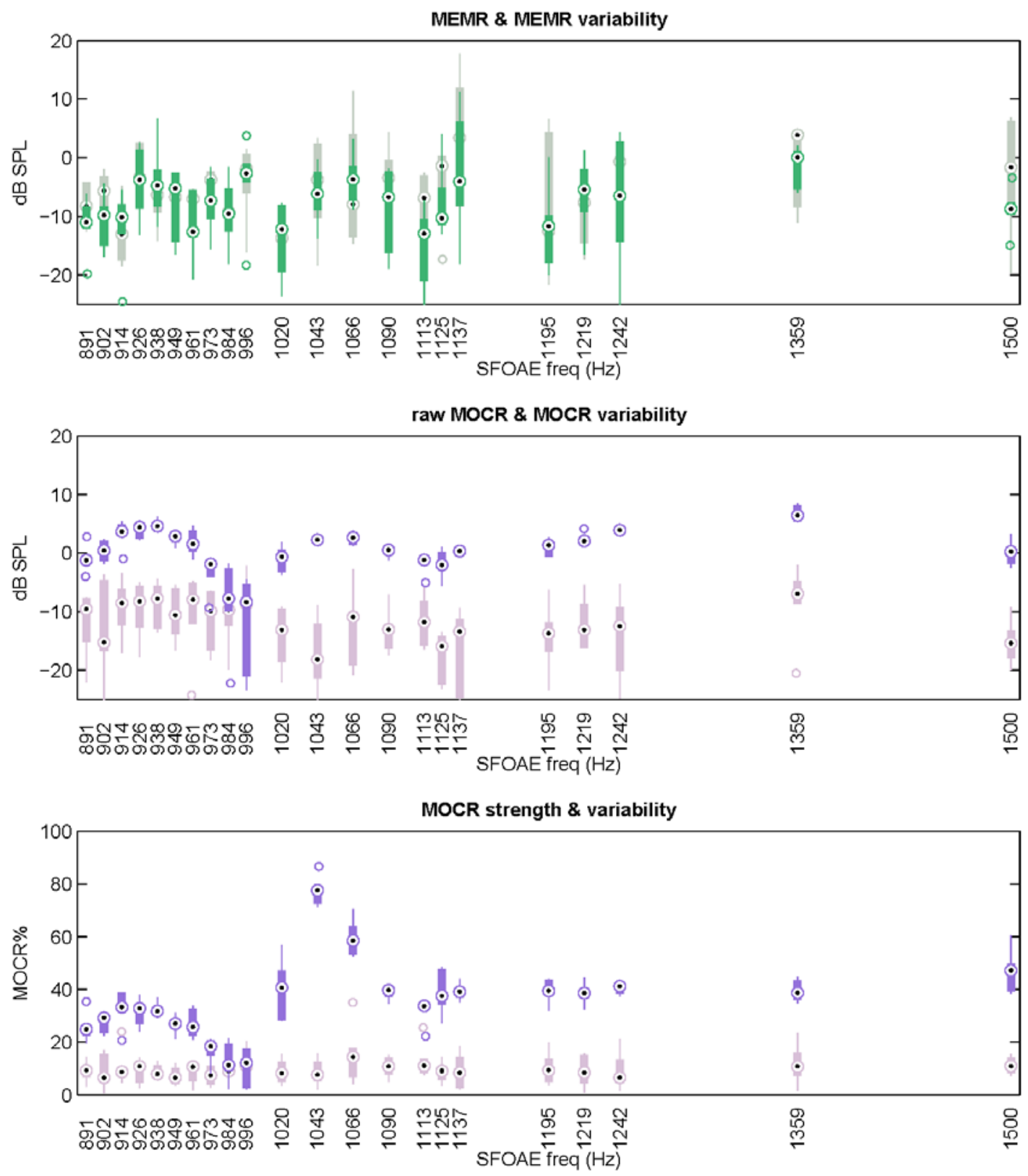

S372R shows high raw MOCR across many frequencies, but the scaled MOCR\% is only moderately strong, and varies from low to high values. This happened for the sub-optimal test frequencies in the " $1 \mathrm{kHz}$ " band where the OAE spectrum was in a null and below our usual level for testing. Thus when normalizing by OAE amplitude, the MOCR\% was overly inflated or diminished. The MEMR tests showed some good test frequencies where variability was low, and others where it was high. High MEMR variability was not necessarily associated with MOCR measurements that were outliers. 


\section{Subject 395L}
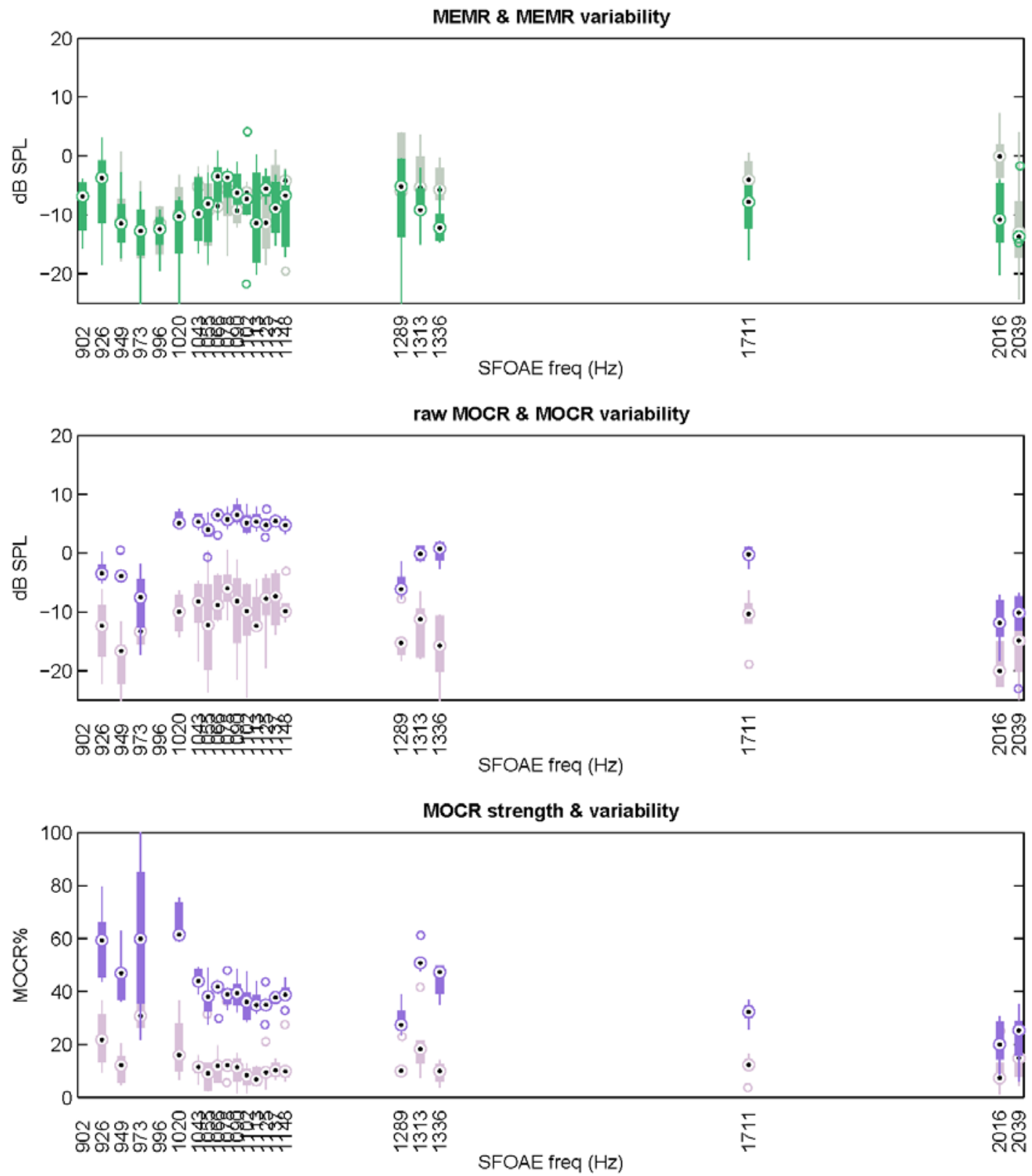

S395L shows a strong MOCR response. At the lowest frequencies, it gets quite variable, due to OAEs with poor SNR. The MEMR measurement shows no reflex present and low variability, giving us confidence in the MOCR results, especially because the raw MOCR is orders of magnitude larger than any MEMR activity. 


\section{Subject 396L}
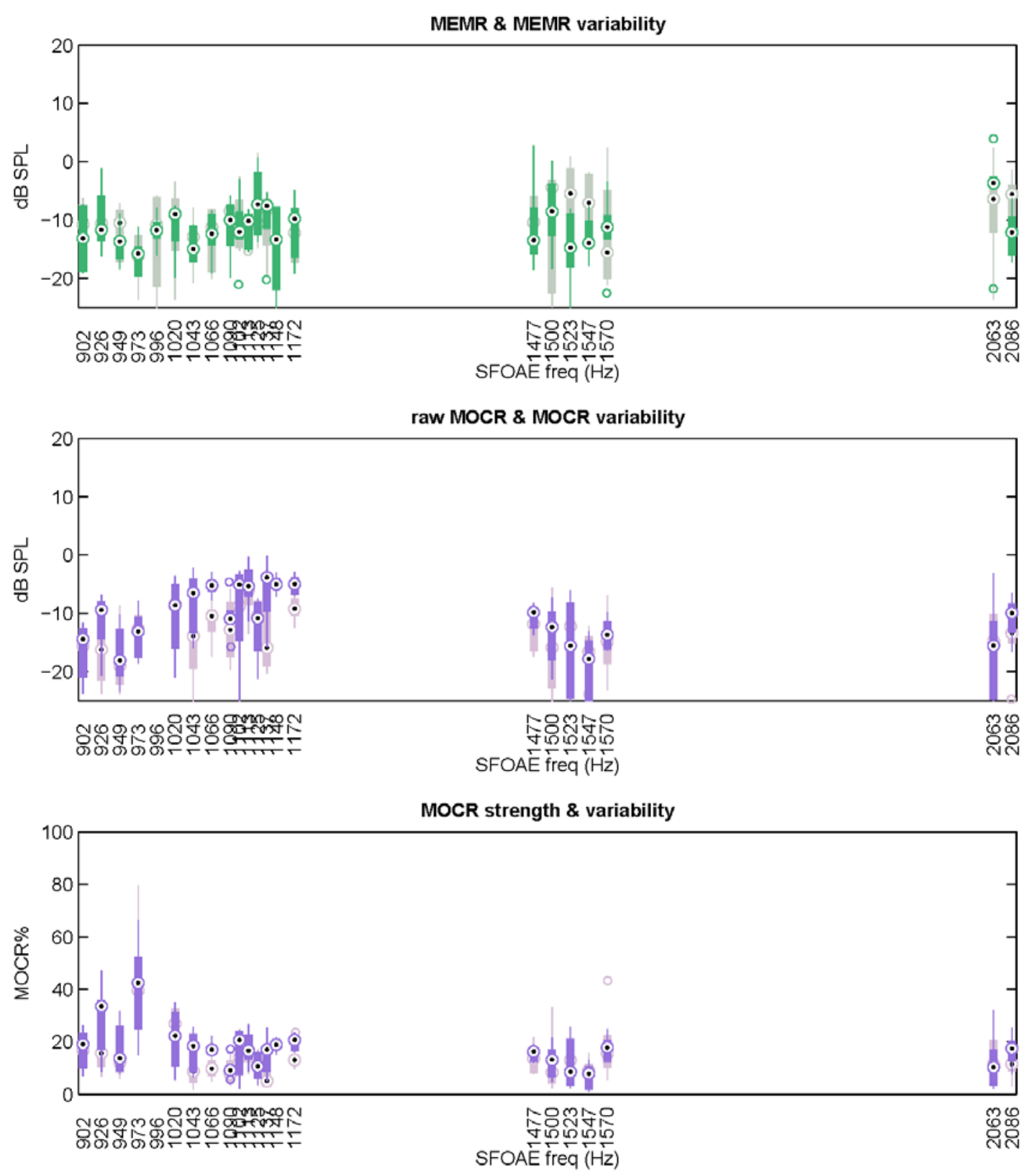

S396L shows a low MOCR response that is mostly undetectable from the inherent test variability. The lowest frequencies show less MOCR activity because they are measured at suboptimal points on the OAE spectrum. The MEMR and variability is also low, suggesting that the MOCR is not diminished due to MEMR activity. 


\section{Subject 398R}

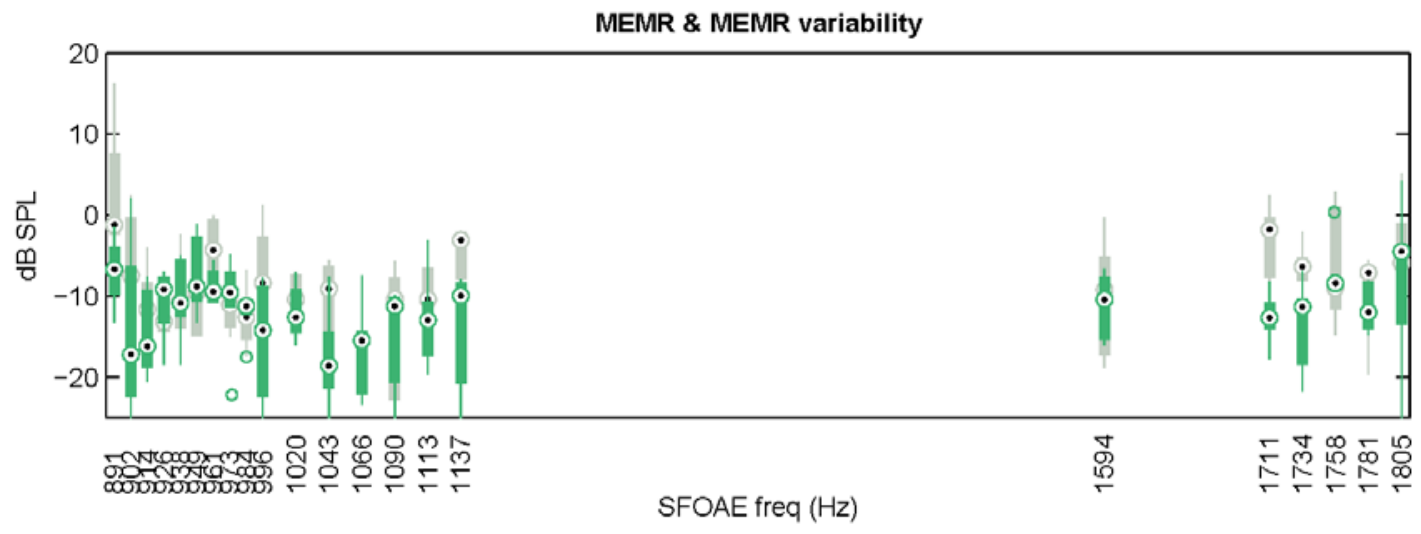

raw MOCR \& MOCR variability

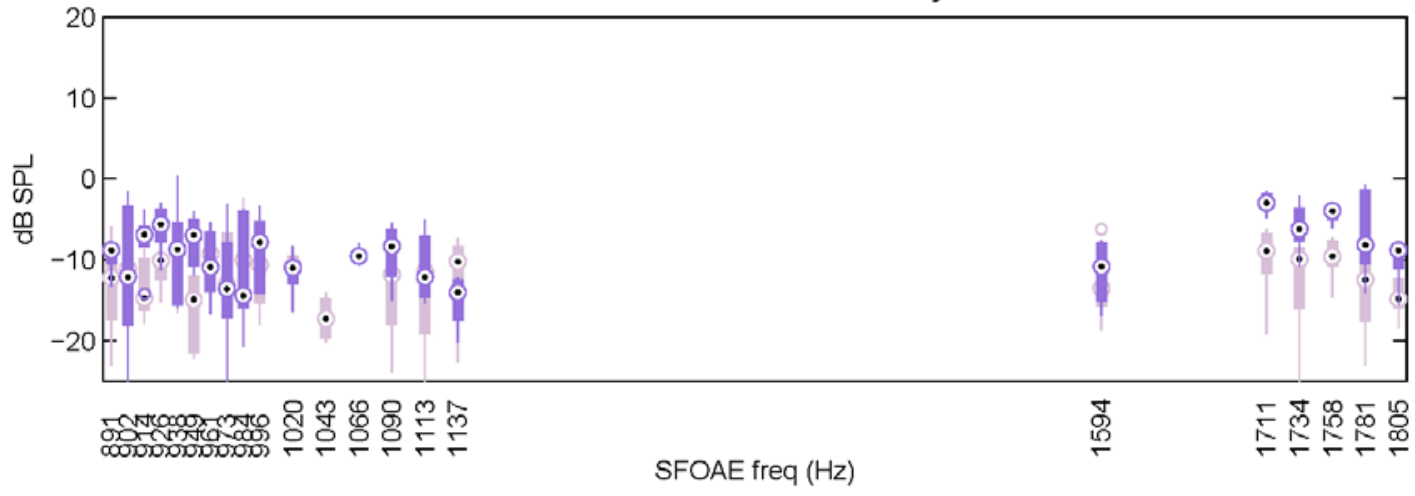

MOCR strength \& variability

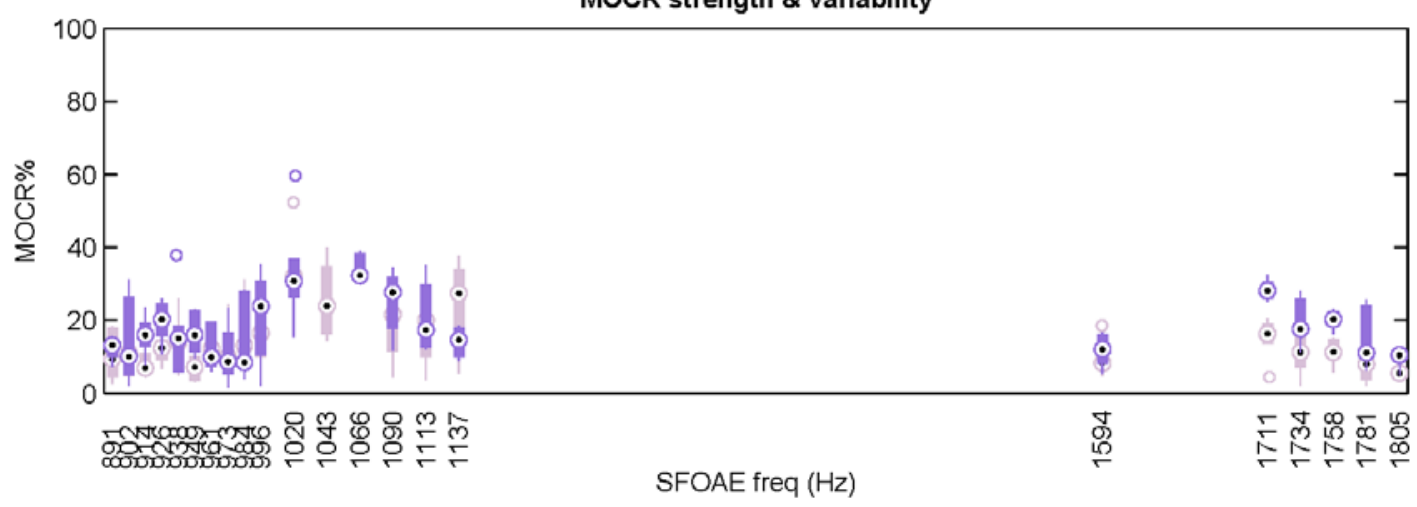

S398R MOCR is low and mostly undetectable from test variability. MEMR activity and variability is also mostly low, and unrelated to the MOCR frequencies that are more variable. 


\section{Subject 399L}
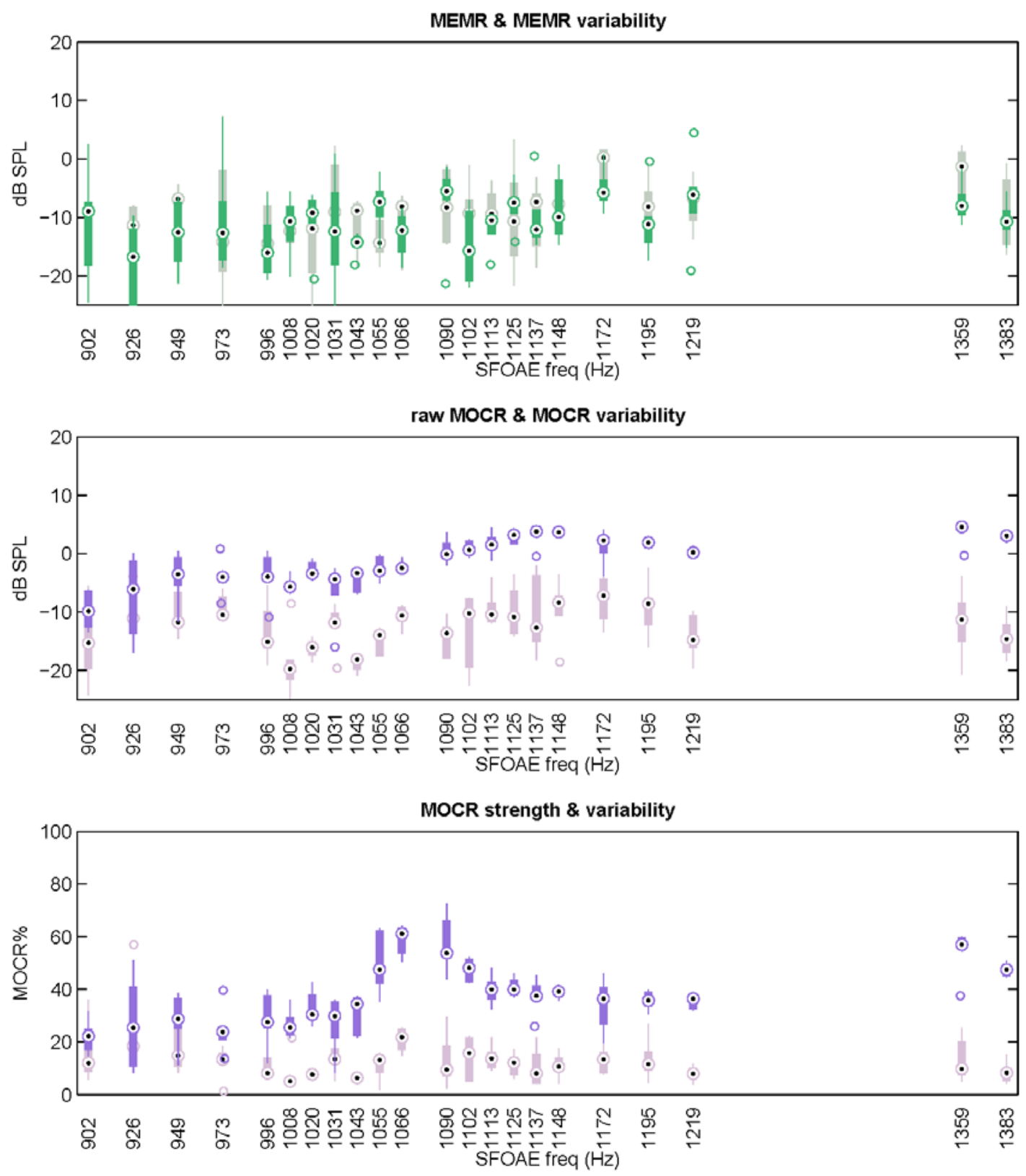

S399L has a moderately high MOCR response that is clearly separable from test variability at most frequencies. The more variable section around $1055-1102 \mathrm{~Hz}$ is due to low level OAEs. MEMR activity and associated variability is mostly low and is much lower than the raw MOCR, giving confidence that the MOCR results are unaffected by the MEMR. 


\section{Subject 400R}
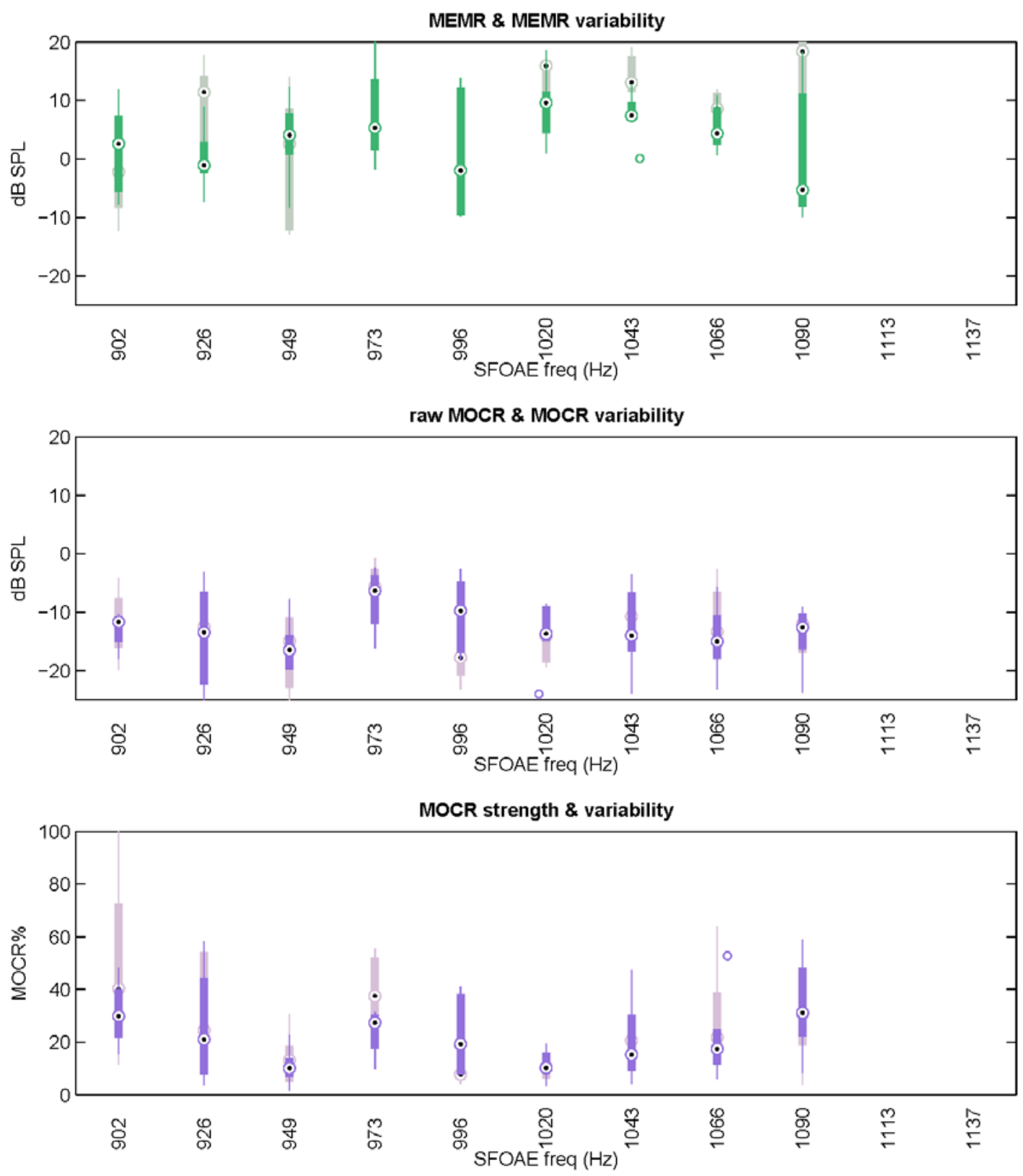

S400R was tested only in the $1 \mathrm{kHz}$ band. MOCR activity was negligible and variability was high at some frequencies. MEMR activity was potentially masked by high MEMR variability, and potentially a masked MEMR could be much larger in magnitude than the raw MOCR. These MOCR results could be affected by MEMR activity, leading to more variable results. 


\section{Subject 401R}
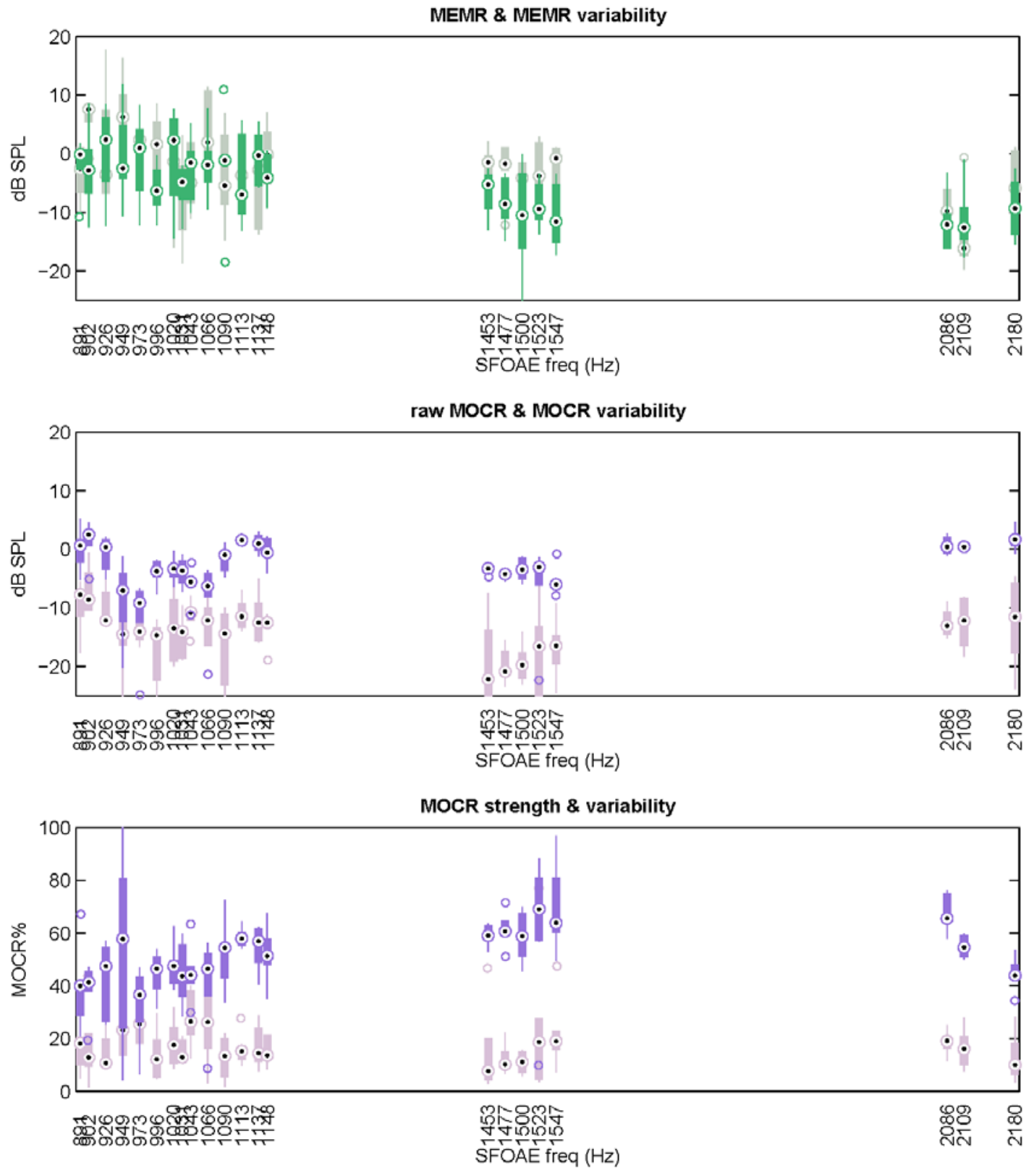

S401R has a strong but MOCR variable response (both within a frequency, indicated by long purple bars, and across frequency); however, it is clearly separable from test variability. The MEMR activity is also quite variable, and a similar order of magnitude as the raw MOCR. It is possible that the variability seen in the MOCR is partly due to MEMR activity. However, this ear also has relatively low OAEs, so some of the MOCR strength variability is likely due to poor SNR. 


\section{Subject 402L}
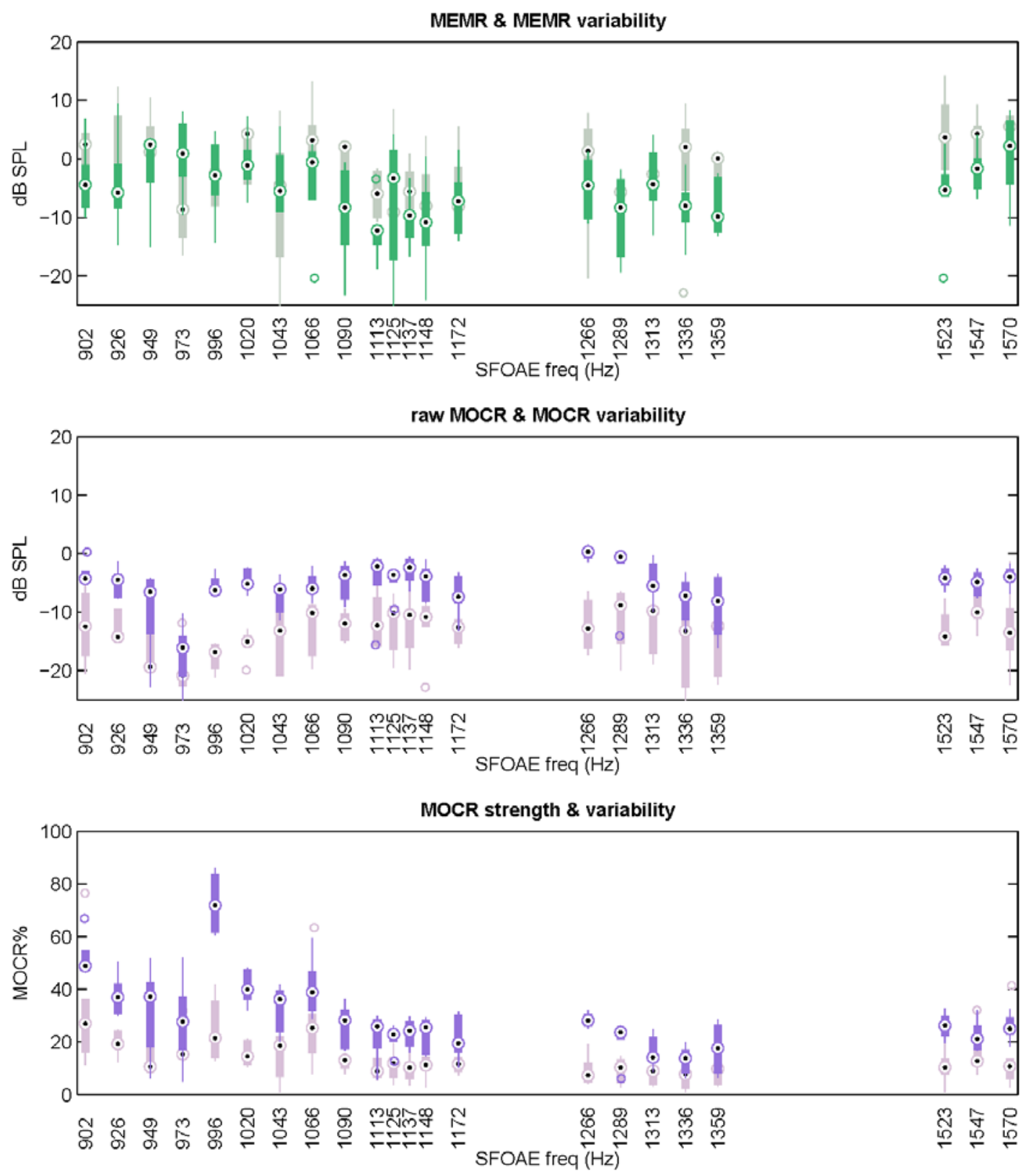

S402L has a mostly moderate, detectable, MOCR response that varies across frequency. The MEMR variability is moderately high, and potentially could be contributing to some MOCR variability. The high MOCR at $996 \mathrm{~Hz}$ is most likely due to a low-level OAE. 


\section{Subject 402R}
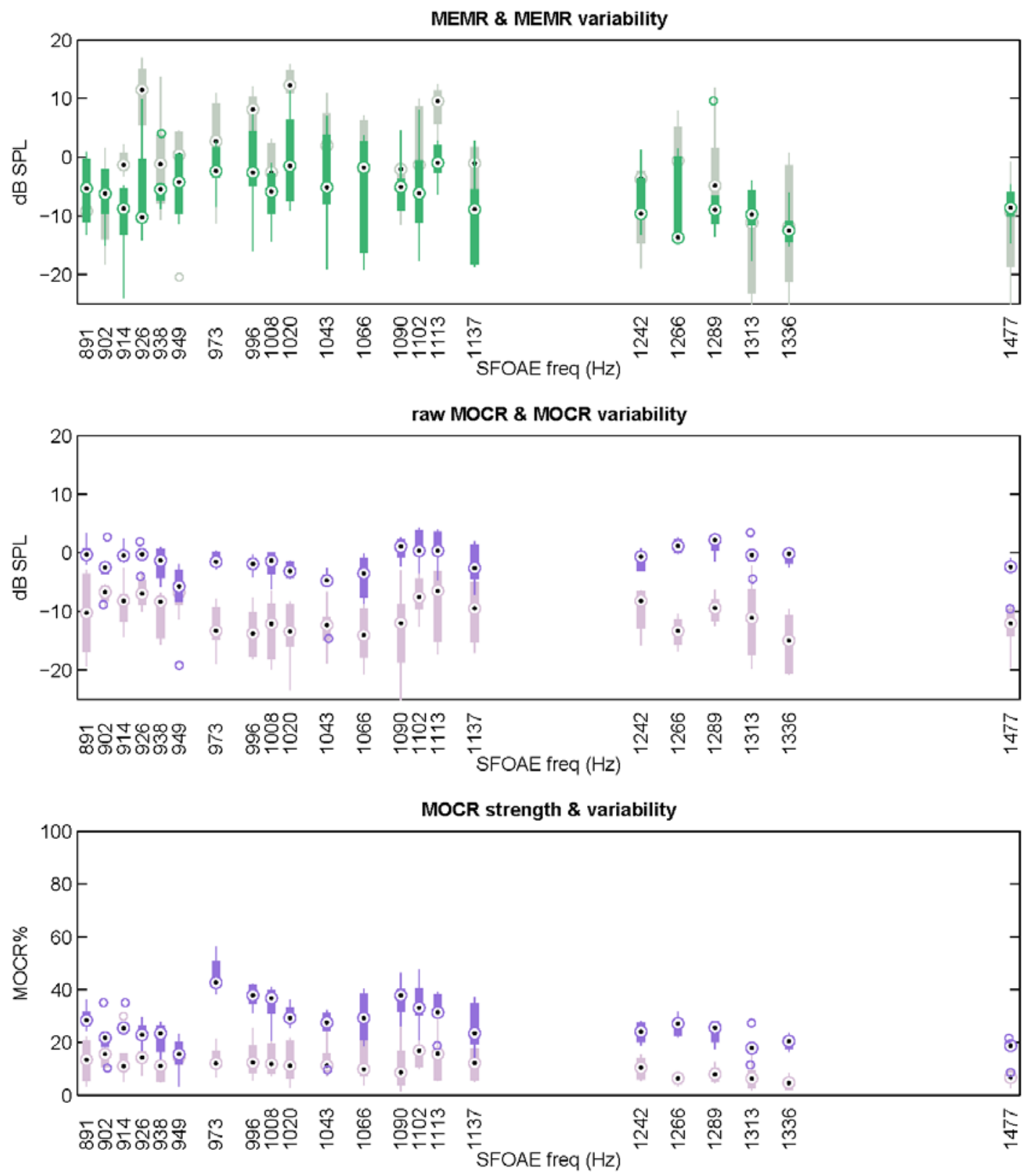

S402R shows a detectable moderately-low MOCR response. Outliers are associated with dips in the OAE spectrum, where measurements were made at suboptimal frequencies. The MEMR variability is quite high and at a similar level to the raw MOCR, however, and potentially could be masking MEMR activity. MOCR\% is similar across frequency, and is not obviously associated with differing degrees of MEMR variability. 


\section{Subject 411L}
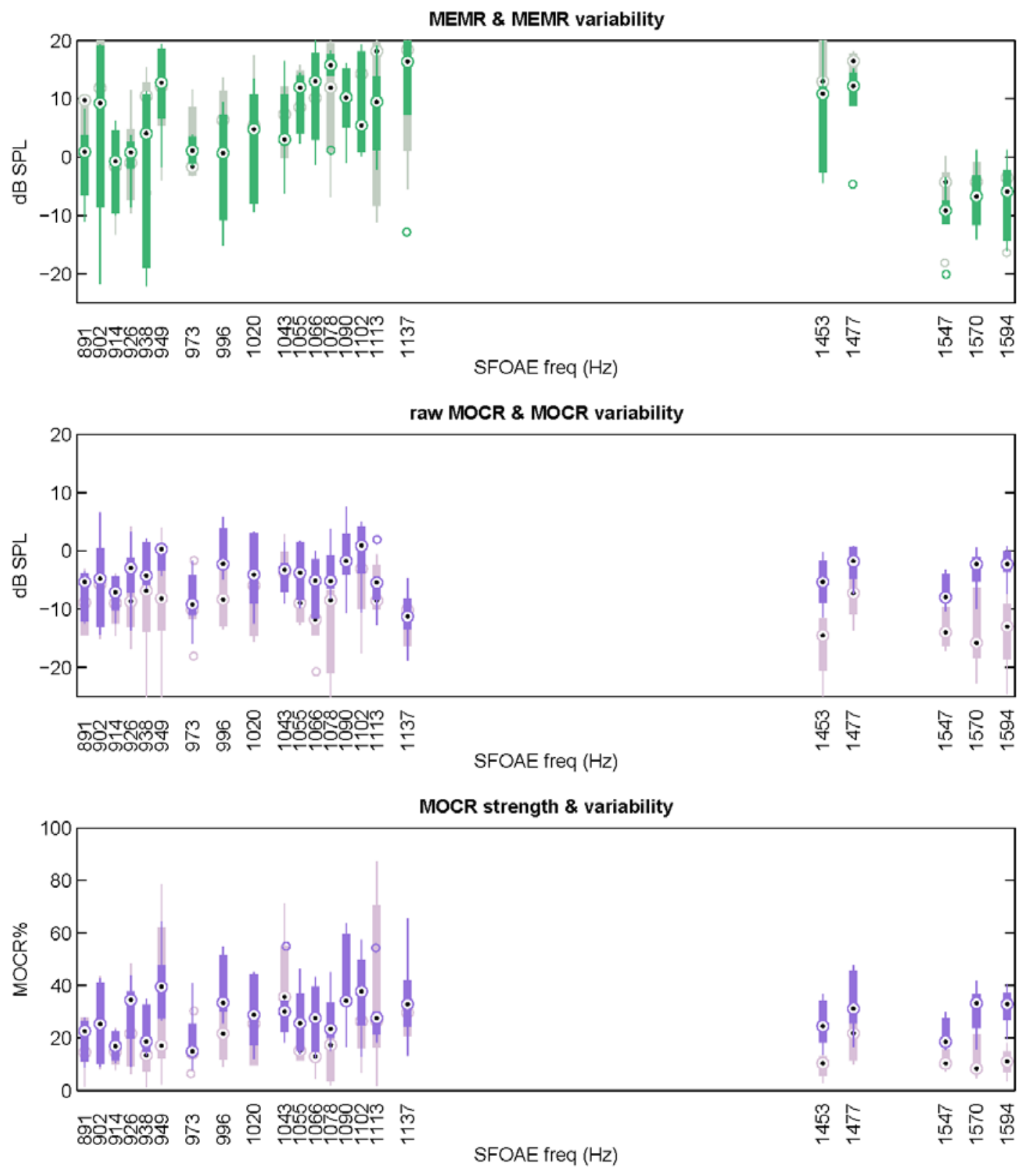

S411L has mostly undetectable MOCR with high inherent test variability. The MEMR is also highly variable, and could potentially be masking MEMR activity that is at least as strong as the raw MOCR. 